


\section{B U L L E T I N \\ DE CORRESPONDANCE HELLÉNIQUE}

\section{VOLUME 141 \\ 2017}

FASCICULE 1

É C O LE FRANÇAISE D'ATH ÈNES 


\section{B U L L E T I N \\ DE CORRESPONDANCE \\ HELLÉNIQUE}

publié depuis 1877

\section{$141.1 \bullet 2017$}

Comité de rédaction : Alexandre FARnoux, directeur

Bertrand Grandsagne, responsable des publications

\section{COMITÉ DE LECTURE}

Le comité de lecture de l'École française d'Athènes est composé de trois membres de droit et de neuf membres désignés par le conseil scientifique sur proposition du directeur. Sa composition actuelle est la suivante (conseil scientifique de l'École française d'Athènes du 25 juin 2012) :

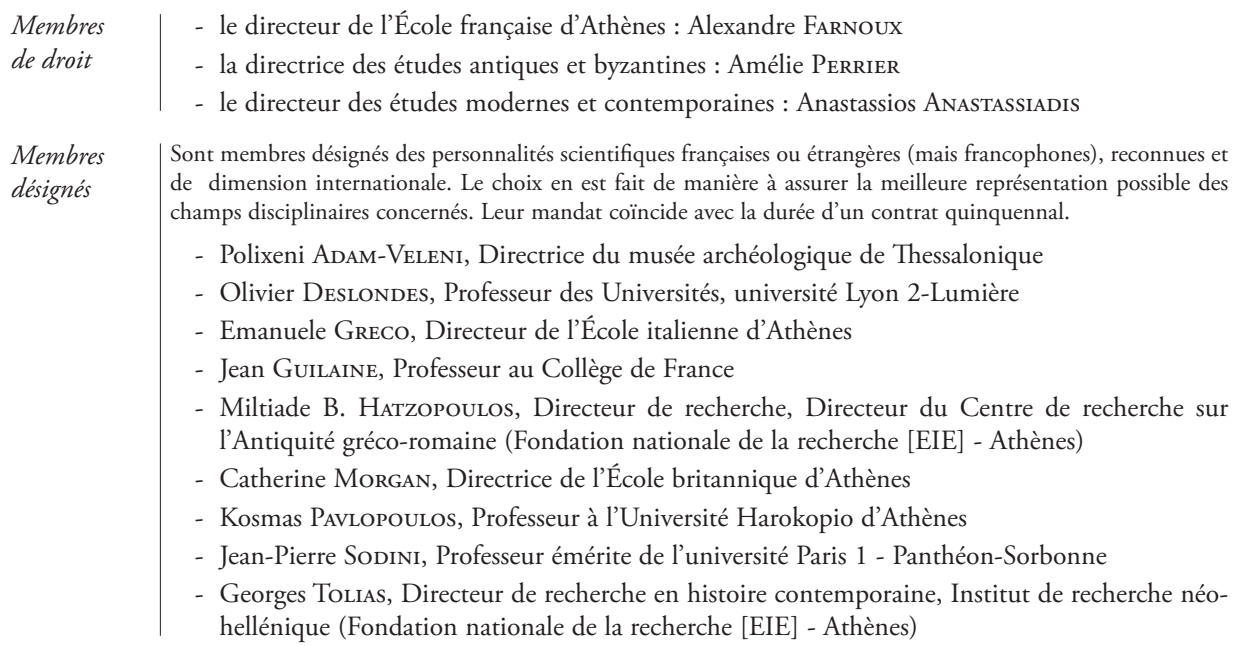

Le comité de lecture fait appel en tant que de besoin à des experts extérieurs.

Révision des textes : EFA

Traduction et révision des résumés en grec : Pavlos Karvonis

Traduction et révision des résumés en anglais : Freya Evenson

Réalisation en PAO : Scuola Tipografica S. Pio X (Rome, Italie)

Impression et reliure : Corlet Imprimeur (Condé-sur-Noireau, France)

(C) École française d'Athènes, 2018

6, rue Didotou GR - 10680 Athènes www.efa.gr

ISBN 978-2-86958-298-9

ISSN 0007-4217

Reproduction et traduction, même partielles, interdites sans l'autorisation de l'éditeur pour tous pays, y compris les États-Unis. 


\section{AVIS AUX LECTEURS}

Le fascicule $2 \mathrm{du}$ Bulletin de correspondance hellénique achève aujourd'hui sa mutation. On y lisait depuis 1920 la chronique des travaux archéologiques réalisés en Grèce et à Chypre. Pour mieux faire circuler une documentation toujours plus importante et en permettre une meilleure utilisation, l'EFA a décidé en 2009, en collaboration avec la British School at Athens, de faire de la Chronique une publication en ligne, sur une page dédiée (chronique.efa.gr). Les chiffres de la fréquentation de cette page (en 2017, 5470 visiteurs et plus de 46000 consultations) montrent que ce choix a satisfait les attentes d'un public toujours plus mobile et plus demandeur de documentation en ligne.

Forte de cette première expérience positive, l'École veut aujourd'hui améliorer la diffusion des "Rapports sur les travaux de l'École française d'Athènes ». Une partie du fascicule 2 du Bulletin de correspondance hellénique est traditionnellement consacrée à la présentation de l'activité archéologique de l'École française d'Athènes. On trouve encore dans la livraison de 2016 ce rapport composé des textes proposés par les responsables de missions ou de programmes. À partir de cette année, il sera mis en ligne sur une page dédiée, dans un format éditorial spécifique et associé au réseau des Écoles françaises à l'étranger. Ce changement de support permettra une plus grande rapidité de publication, une visibilité accrue de l'institution et la mise à disposition d'une documentation plus abondante et en couleurs. Par ce choix, l'École a la volonté de toucher un plus grand nombre de lecteurs et de mieux faire circuler l'information scientifique à une époque où les supports (blogs, cahiers numériques etc.) se multiplient.

Le basculement des Rapports vers un support numérique permet de consacrer désormais les deux fascicules de la revue aux articles de fond et de synthèse. Il renouvelle ainsi, en lui donnant une nouvelle dimension, la vocation première du Bulletin de correspondance hellénique. 


\section{SOMMAIRE DE LA LIVRAISON}

\section{Maia PoMAdèRe}

Classes d'âge et rites de passage: des catégories problématiques pour le monde égéen à l'Âge du Bronze

Raphaël Orgeolet et alii

Kirrha 2008-2015: un bilan d'étape, 1. La fouille et les structures archéologiques......

Christophe Flament

Etude sur la chronologie des archontats de Damasias à Athènes et de la première guerre sacrée à Delphes.

Sylvian Fachard, Thierry Theurillat, Athanasia Psalti, Delphine Ackermann et Denis Knoepfler

La Nécropole du Canal à Éétrie: topographie et inscriptions

Anne-Marie Guimier-Sorbets

Des emblemata - pinakes déliens : iconographie, technique, usage

Patrice Hamon

Études d'épigraphie thasienne, $V$. Théores et archontes thasiens de l'époque hellénistique et impériale: du simple au double.

Lavdosh JaUpaJ

Une tombe hellénistique de Gjerbës (Albanie) : un marqueur culturel?

Jean-Baptiste CAYLA

Antoine, Cléopâtre, et les technites dionysiaques à Chypre.

Paul ERnSt

Le logement des particuliers romains dans la Grèce égéenne aux II et I I av. J.-C., d'après les sources textuelles

Véronique FrançoIs

Fragments d'histoire II : la vaisselle de table et du quotidien à Nicosie au lendemain de la conquête ottomane.

Edhem Eldem

Et si Diane n'était pas lesbienne? Biographie critique d'une statue(tte) d'Artémis du Musée archéologique d'Istanbul.. 


\title{
La Nécropole du Canal à Érétrie : topographie et inscriptions
}

\author{
Sylvian Fachard, Thierry Theurillat, Athanasia Psalti, Delphine Ackermann \\ et Denis Knoepfler
}

RÉsumÉ L'automne 2009 vit de violentes pluies s'abattre sur l'Eubée. À Érétrie, plusieurs tombes et stèles funéraires apparurent dans le lit du ruisseau bordant le site à l'Ouest. Ces découvertes apportent un éclairage bienvenu sur les nécropoles érétriennes, encore mal connues. Par ailleurs, plusieurs murs antiques furent documentés : si certains s'apparentent aux murs du canal archä̈que déjà relevés à la Porte de l'Ouest, d'autres datent de l'époque classique-hellénistique et ouvrent de nouvelles pistes de recherche concernant l'étude de l'enceinte et la topographie de ce secteur. L'étude des stèles funéraires, dont la date va de l'extrême fin du v $v^{\mathrm{e}}$ à la seconde moitié du III ${ }^{\mathrm{e}}$ s., donne lieu à des observations nouvelles sur des sujets aussi variés que les techniques de taille, la typologie et l'iconographie des monuments, l'onomastique et les composantes de la population érétrienne.

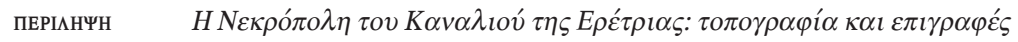

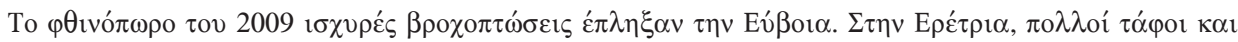

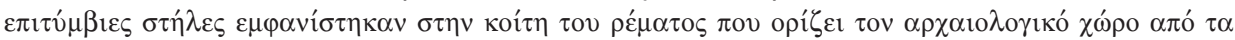

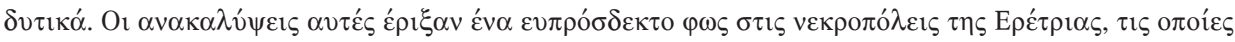

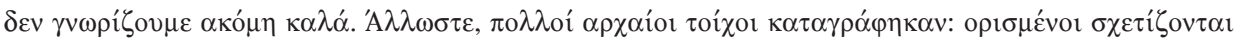

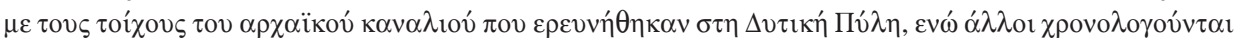

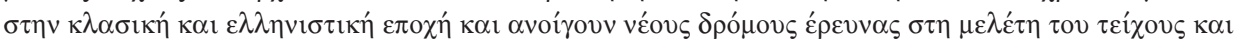

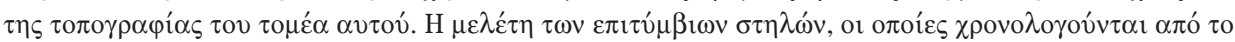

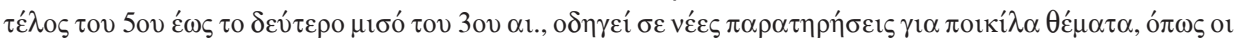

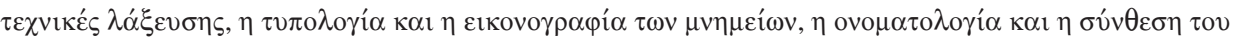

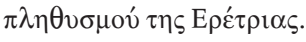

SUMmary The Cemetery of the Canal at Eretria: topography and inscriptions

In the autumn of 2009, Euboea was inundated by heavy rainfall. In Eretria, many tombs and funerary stelae appeared in the bed of the torrent that delimits the archaeological site on the west. These discoveries shed welcome light on the cemeteries of Eretria, which are still little understood. Additionally, many ancient walls were recorded: several are associated with the walls of the Archaic canal previously explored at the West Gate, while others date to the Classical and Hellenistic period and provide new avenues of research in the study of the fortification wall and the topography of this sector. An examination of the funerary stelae, which date from the end of the 5th to the second half of the 3rd century, leads to new observations on a variety of subjects, including carving techniques, typology and iconography of the monuments, and onomastics and the composition of the population at Eretria. 
À PROPOS DES AUTEURS

Sylvian Fachard, A. W. Mellon Professor of Classical Studies, American School of Classical Studies at Athens, ancien Collaborateur scientifique FNS au Département des sciences de l'Antiquité de l'Université de Genève, ancien Secrétaire scientifique de l'École suisse d'archéologie en Grèce; Thierry Theurillat, Secrétaire scientifique en Suisse de l'École suisse d'archéologie en Grèce; Athanasia Psalti, Directrice de l'Éphorie de Phocide, ancienne épimélète de l'Éphorie d'Eubée, responsable du site d'Érétrie; Delphine Ackermann, maître de conférences en histoire grecque à l'université de Poitiers, collaboratrice scientifique de l'École suisse d'archéologie en Grèce et ancienne membre étrangère de l'École française d'Athènes et Denis Knoepfler, ancien membre étranger de l'EFA, professeur honoraire de l'Université de Neuchâtel, professeur émérite au Collège de France (chaire d'épigraphie et d'histoire des cités grecques), directeur scientifique des fouilles gréco-suisses d'Amarynthos (ESAG), associé étranger de l'Académie des Inscriptions et Belles-Lettres. 
Des intempéries d'une rare violence tombèrent sur l'Eubée en septembre 2009. Les 11 et $12 \mathrm{du}$ mois, $160 \mathrm{~mm}$ de pluie furent enregistrés à Chalcis, ce qui correspond à près d'un quart des précipitations de l'ensemble de l'année 2009 (668,5 mm). Dans la partie centrale de l'île, les dégâts furent considérables. Outre une perte humaine à déplorer, plusieurs ponts et barrages furent emportés, près de 200 hectares de cultures détruits et 500 maisons inondées ${ }^{1}$.

À Érétrie, c'est un véritable déluge qui s'abattit sur la ville ${ }^{2}$. En l'espace de quelques heures, environ 15\% de l'ensemble des précipitations de l'année 2009 se déversa sur le site antique. Les rues de la ville se muèrent rapidement en torrents de boue ${ }^{3}$. Le cours d'eau saisonnier qui borde les murailles occidentales de la ville antique (et qui ne fait parfois, nous le verrons, qu'un avec le lit du cours antique), gonflé de boue, de troncs d'arbre et de détritus modernes, déborda et emporta dans la mer des pans entiers de berges mal terrassées. Le secteur allant de la Porte de l'Ouest au Nord de la cité antique fut le plus touché : sur plus de 200 mètres (carrés A-B/500-300 du plan de référence, fig. I), le torrent emporta plusieurs centaines de mètres cubes de terre, ravinant la route asphaltée et provoquant l'effondrement de plusieurs murs de terrasse modernes. Une inspection dans le lit défoncé du cours d'eau révéla de nombreuses sépultures éventrées ainsi que 29 stèles inscrites et une dizaine de fragments de marbre appartenant à des monuments funéraires (fig. 2). Dans ces circonstances dramatiques, l'Éphorie des Antiquités d'Eubée invita l'École suisse d'archéologie en Grèce à mener de concert une fouille de sauvetage. Au cours de cette opération, il fut possible de fouiller, relever et documenter 22 tombes et plusieurs tronçons de murs antiques ${ }^{4}$.

1. L’officier de police Nikos Triantaphylou perdit la vie dans des circonstances tragiques au pont de Stomio, près de Kymi. Il avait hébergé notre équipe de fouille à Platania, lors de notre campagne de relevés architecturaux en 2005 à Kotylaion, dème fortifié de l'Érétriade. Les rivières du Lélante, du Sarandapotamos (vallée d'Amarynthos) et du Manikiatis débordèrent de leur lit, se transformant en torrents de

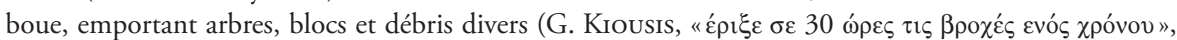
Eleutherotypia, 14 septembre 2009). Dans la vallée du Manikiatis, le phénomène fut amplifié par les terribles incendies de 2007 qui avaient alors dévasté la couverture végétale de la région, en particulier les pentes les plus sensibles à l'érosion ( $A K 51$ [2008], p. 146). Sur la géographie de cette région, voir S. FACHARD, La défense du territoire d'Érétrie : étude de la chora et de ses fortifications, Eretria XXI (2012), p. 35-46.

2. Selon nos estimations, le volume des intempéries fut plus important qu’à Chalcis $(99,6 \mathrm{~mm})$, dépassant les $100 \mathrm{~mm}$. Le terme technique de "déluge» s'applique lorsqu'environ $100 \mathrm{~mm}$ de pluie sont enregistrés lors d'un même événement, voir A. T. Grove, O. Rackham, The Nature of Mediterranean Europe: An Ecological History (2003), p. 247.

3. La fouille que nous conduisions dans la parcelle O.T. 737, où la profondeur des sondages atteignait plus de $2 \mathrm{~m}$, fut entièrement recouverte par les eaux boueuses en provenance des pentes de l'acropole.

4. Les travaux débutèrent le lendemain sous la direction de S. Fachard et d'A. Psalti, secondés par cinq ouvriers et bientôt rejoints par Th. Theurillat. Les opérations de terrain furent achevées le vendredi 2 octobre. Les auteurs souhaitent remercier M. Duret, G. Ackermann et M. Glaus pour leur aide 


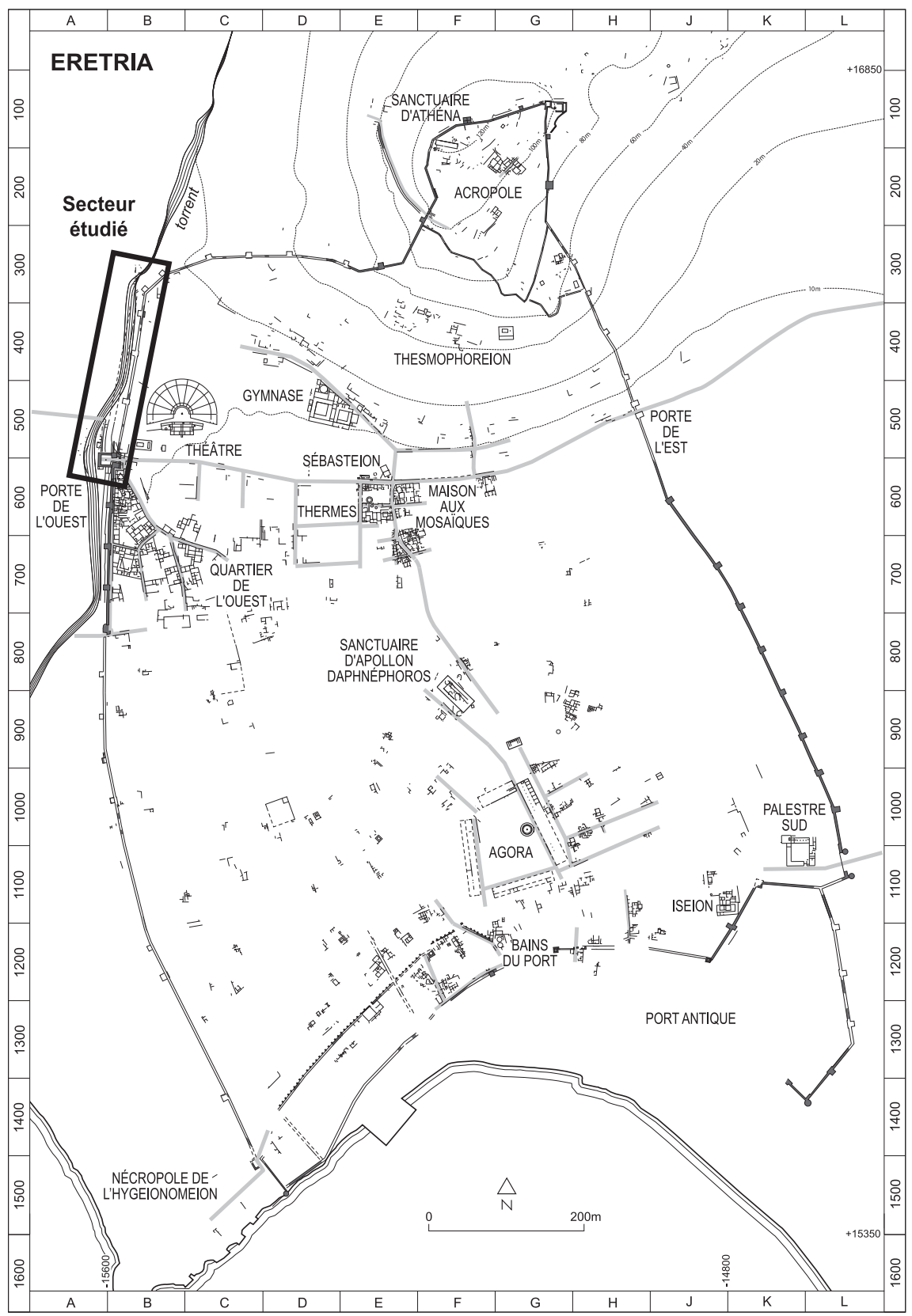

Fig. I — Plan d'Érétrie (ESAG, Th. Theurillat). 


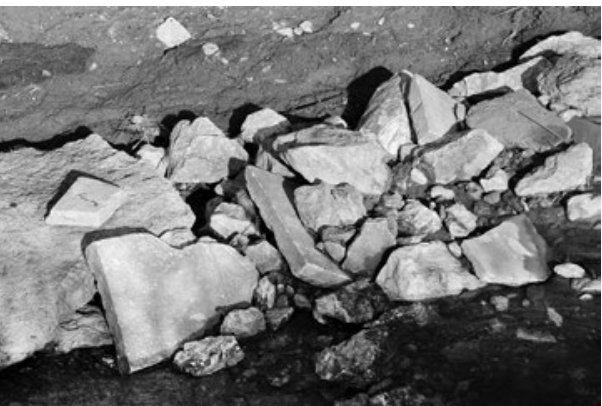

Fig. 2 - Stèles funéraires découvertes dans le cours d'eau suite au déluge du 12 septembre 2009 (cl. ESAG, S. Fachard).
Les lignes qui suivent ne prétendent pas à l'exhaustivité, mais ont pour but de présenter la topographie du secteur, de décrire et dater les principales structures antiques mises au jour et de présenter un premier catalogue des tombes et du matériel qu'elles contenaient. Cette section archéologique sert d'introduction à l'étude des stèles funéraires qui forme le corps de la seconde partie du présent article.

\section{LE CONTEXTE ARCHÉOLOGIQUE (S. Fachard, Th. Theurillat)}

\section{1. LA PROBlÉMATIQUe DU COURS D'EAU SAISONNIER}

À quelques variations près, le cours d'eau saisonnier qui déborda de son lit en 2009 n'est autre que le descendant du cours antique qui, tributaire de plusieurs ruisseaux et thalwegs qui prennent leur source dans le massif du Voudochi, bordait la ville à l'Ouest. Son tracé et son histoire (durant l'Holocène) sont aujourd'hui mieux documentés, grâce aux fouilles archéologiques et aux récentes études géoarchéologiques ${ }^{5}$. D'apparence modeste (il ne saurait être qualifié de « rivière» et demeure anonyme), ce cours d'eau est pourtant à l'origine de la plaine côtière d'origine deltaïque sur laquelle s'implanta le premier habitat au début de l'Âge du Bronze.

En effet, il est désormais établi que l'environnement du site d'Érétrie fut très mobile d'un point de vue géomorphologique, ce qui posa un défi majeur aux habitants, qui y apportèrent des réponses variées ${ }^{6}$. Les mesures les plus spectaculaires furent prises dans les premières décennies du viI ${ }^{\mathrm{e}}{ }^{7}{ }^{7}$, lorsqu'il fut décidé d'assainir le delta en endiguant le

ponctuelle, ainsi que les ouvriers G. Sarapis, E. Fotiadis et L. Kouroupis. Voir S. Fachard, A. Psalti, Th. Theurillat, "Fouilles d'urgence dans le Canal et la nécropole occidentale d'Érétrie ", AK53 (2010), p. 151-155.

5. M. Ghilardi, S. Müller-Celka, Th. Theurillat, S. Fachard, M. Vacchi, "Évolution des paysages et histoire de l'occupation d'Érétrie (Eubée, Grèce). Du Bronze Ancien à l'époque romaine», dans M. Ghilardi, L. Lespez, F. Leandri, S. Fachard, C. Bressy-Leandry (éds), Géoarchéologie des îles de la Méditerranée (2016), p. 149-163.

6. Ibid.

7. Sauf mention contraire, les dates indiquées sont avant J.-C. 
bras oriental du cours d'eau en direction du marais oriental. Un mur de soutènement massif fut alors érigé, composé de blocs de calcaire grossiers et atteignant par endroits plusieurs mètres de hauteur. Dès sa découverte dans les années 1960, la monumentalité de l'ouvrage frappa les esprits, si bien que ce mur de soutènement, par endroits doublé d'un mur parallèle, fut interprété comme un mur de fortification. Cette hypothèse, contredite par les récentes études archéologiques et topographiques ${ }^{8}$, continue néanmoins à jouir d'une certaine notoriété'. Or, l'interprétation d'un mur de berge trouve sa justification dans une série d'épisodes torrentiels bien documentés par les cartes anciennes et les carottages géologiques, ce qu'illustre encore l'épisode de 2009. Des parallèles pour la Grèce permettent de se faire une meilleure idée de tels phénomènes. Lors d'un déluge considéré comme mineur (qui survient tous les cinq ans environ en moyenne), une rivière peut emporter jusqu'à 20 fois plus de sédiments pendant le seul mois du déluge que durant le reste de l'année, et un déluge de $100 \mathrm{~mm}$, comme celui enregistré en 2009, peut produire davantage d'érosion que dix précipitations distinctes de $10 \mathrm{~mm}^{10}$. À Érétrie, les études géomorphologiques ont permis de documenter une crise détritique considérable amorcée lors de la réoccupation du site au $\mathrm{Ix}^{\mathrm{e}} \mathrm{s} .{ }^{11}$. Cette crise entraîna une série d'épisodes torrentiels, contre lesquels les habitants décidèrent de se prémunir en construisant une succession de murs de berge dès le début du viI ${ }^{\mathrm{e}} \mathrm{s}$. L'environnement ne se stabilisa progressivement qu'à partir du milieu du $\mathrm{VI}^{\mathrm{e}} \mathrm{s}$., grâce à la mise en place de nouvelles mesures édilitaires, qui modifièrent durablement la configuration du site : vers 550, le canal oriental qui traversait l'habitat antique fut définitivement condamné à la hauteur de la future Porte de l'Ouest par un solide massif faisant barrage. Le cours d'eau saisonnier s'écoulait désormais en ligne droite vers la mer. Les berges du cours d'eau furent également renforcées par des murs de soutènement, réalisés dans un appareil fruste mais dont la solidité éprouvée fut encore confirmée lors du déluge de 2009. C'est en retrait du mur de soutènement oriental que s'éleva la première muraille archaïque d'Érétrie, avant de servir de terre-plein pour la muraille classique. Élément naturel dévastateur dès la fondation de la cité, le cours d'eau saisonnier fut donc progressivement contenu par les Érétriens dès le viI ${ }^{e}$ s., avant d'être définitivement endigué au milieu du $\mathrm{VI}^{\mathrm{e}} \mathrm{s}$.

8. La discussion est résumée par S. FACHARD, "L'enceinte urbaine d'Érétrie : un état de la question », $A K 47$ (2004), p. 91-109.

9. R. Frederiksen, Greek City Walls of the Archaic Period, 900-480 BC (2011), p. 138-141.

10. A. T. Grove, O. Rackham (n. 2), p. 30-36.

11. Les causes en sont encore inconnues. Sans écarter des facteurs naturels, on ne peut exclure des facteurs anthropiques, liant ces phénomènes d'alluvionnement avec un déboisement accru des pentes du Voudochi provoqué notamment par un accroissement de la population. Voir M. GHILARdi et al. (n. 5). 


\section{2. LES MURS DE BERGE ARCHAÏQUES ( $\mathrm{VII}^{\mathrm{e}}-\mathrm{VI}^{\mathrm{e}} \mathbf{s}$.)}

La campagne de 2009 a permis de documenter et de relever plusieurs murs que l'on peut associer aux murs de berge archaïques mis au jour de part et d'autre de la Porte de l'Ouest dans les années 1960. Ces tronçons, dont certains n'étaient pas connus, confirment l'endiguement précoce du cours d'eau au $\mathrm{VII}^{\mathrm{e}} \mathrm{s}$., ainsi que l'ampleur tout à fait considérable de cette entreprise.

\section{Le mur M3}

Le parement d'un mur antique de très bonne facture (M3) fut dégagé à $200 \mathrm{~m}$ au Nord de la Porte de l'Ouest. Orienté Nord-Sud, il paraît uniquement parementé à l'Ouest, indiquant qu'il s'agit d'un mur de soutènement. Il est implanté directement sur le rocher naturel, qui a été naturellement poli par les eaux au cours des millénaires. Conservé sur une longueur de $9 \mathrm{~m}$ et une élévation de 1,40 m, M3 est construit dans un appareil irrégulier, aux joints lâches, composé de pierres non travaillées (fig. 3, 4). Il s'agit néanmoins d'une solide construction avec des blocs atteignant parfois $1 \mathrm{~m}$ de hauteur $(100 \times 60 ; 92 \times 82 \mathrm{~cm})$, calés par des éléments plus petits et des moellons. C. Krause rattacha ce mur à la première phase de construction de la Porte de l'Ouest (BP1), soit au VII ${ }^{\mathrm{e}}$ s. ${ }^{12}$. La fouille en 2009 d'une cavité intercalée entre le rocher naturel et l'assise de fondation de M3 a livré un ensemble céramique comprenant plusieurs vases entiers, en majorité des petits vases à boire monochromes parmi lesquels on reconnaît une tasse de type "Phalère", datée du $1^{\text {er }}$ quart du ViI ${ }^{\mathrm{e}}$ s. ${ }^{13}$. Ce terminus post quem confirme la construction précoce des premiers murs de digue du cours d'eau occidental vraisemblablement dans le second quart du VII ${ }^{\mathrm{e}}$ s. ${ }^{14}$.

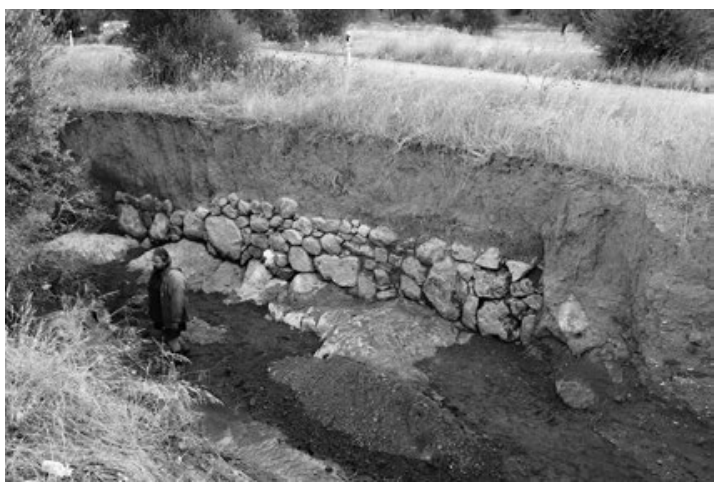

Fig. 3 - Vue du mur M3 (cl. ESAG, S. Fachard).

12. C. Krause, Das Westtor. Ergebnisse der Ausgrabungen 1964-68, Eretria IV (1972), p. 16-21 et fig. 12 et 14.

13. S. Fachard, A. Psalti, Th. Theurillat (n. 4), p. 153 n. 44.

14. C. Krause a proposé les années précédant le milieu du viI ${ }^{\mathrm{e}}$ s. (S. Fachard, A. Psalti, Th. Theurillat [n. 4], p. 153). 


\section{Les murs M8 et M7}

Parmi les nouveaux murs découverts en 2009, deux peuvent être rattachés à l'endiguement archaïque du cours d'eau, sans toutefois pouvoir être placés dans une fourchette chronologique plus précise. On mentionnera d'abord le tronçon de mur M8, orienté Nord-Sud. Conservé sur une longueur de $12 \mathrm{~m}$ en travers du cours d'eau moderne, seule l'assise supérieure est visible en plan, composée d'un parement unique en blocs parfois grossièrement équarris (fig. 4). Il n'a pas pu être fouillé, mais autant que l'on puisse en juger, son mode de construction et les blocs employés rappellent les murs du canal archaïque. Son orientation s'aligne parfaitement sur les murs de berge A et M3. On peut donc avec une certaine assurance rattacher ces trois tronçons à un ouvrage unique, sans pour autant affirmer qu'ils font partie de la même phase de construction - point sur lequel nous reviendrons plus bas.

Le mur M7, en revanche, ne se rattache pas à ce mur de digue oriental, puisqu'il est parementé à l'Est. Il retenait donc la berge occidentale du cours d'eau antique. Conservé sur une longueur de $11 \mathrm{~m}$ (fig. 4), son tracé Nord-Sud est désaxé vers l'Est de près de 3 à $4 \mathrm{~m}$ par rapport au parcours rectiligne du mur de digue orientale A-M3-M8 ${ }^{15}$. Comment concilier ces données a priori contradictoires? M7 est situé dans un méandre du ruisseau moderne vers l'Est; des litages de sable, visibles sur plus de $3 \mathrm{~m}$ de hauteur dans la berge orientale, furent si ravinés durant la crue de 2009, que la route asphaltée s'effondra en partie à cet endroit. Ce "point faible» du dispositif d'endiguement apparaît également sur un plan de $1850^{16}$, où le tracé principal du cours d'eau se sépare en un bras secondaire vers l'Est qui s'écoule au Nord du théâtre pour venir inonder le village moderne. Le mur M7 témoigne très probablement de l'adaptation des premiers murs de berge archaïques à un cadre naturel contraignant, qui dut empêcher d'endiguer le cours d'eau selon un parcours rectiligne (fig. 4). Ce n'est vraisemblablement pas avant le milieu du vi ${ }^{\mathrm{e}}$ s. que la berge orientale fut corrigée, lorsque les Érétriens entreprirent de construire les premières fortifications urbaines dans la plaine. D'importants travaux de terrassement furent alors nécessaires pour protéger le nouveau rempart de briques contre les crues soudaines et incontrôlées ${ }^{17}$. À la hauteur de la nouvelle Porte de l'Ouest, le mur de soutènement

15. Une unique assise est visible en plan, se composant d'un seul parement de gros blocs de calcaire bruts pouvant atteindre $1,30 \times 0,50$ et $0,96 \times 0,74 \mathrm{~m}$. En raison de son mode de construction, une date à l'époque moderne semble a priori exclue; de plus, le mur se trouve au centre du cours d'eau actuel, ce qui démontre qu'il ne s'agit pas d'un mur de berge moderne. Il pourrait au contraire indiquer la limite d'un état ancien du cours d'eau, modifié ultérieurement.

16. F. PAJOr, Eretria - Nea Psara. Eine klassizistische Stadtanlage über der antiken Polis, Eretria XV (2006), pl. 20 , p. 28.

17. Les traces de la muraille archaïque restent ténues, au point que leur existence même fut mise en doute. Il n'y a pas lieu de revenir sur ce débat ici, d'autant qu'aucun élément réellement nouveau n'est venu s'ajouter au dossier depuis le dernier état de la question (voir S. FACHARD [n. 8], p. 94-99). Nous soulignerons, 


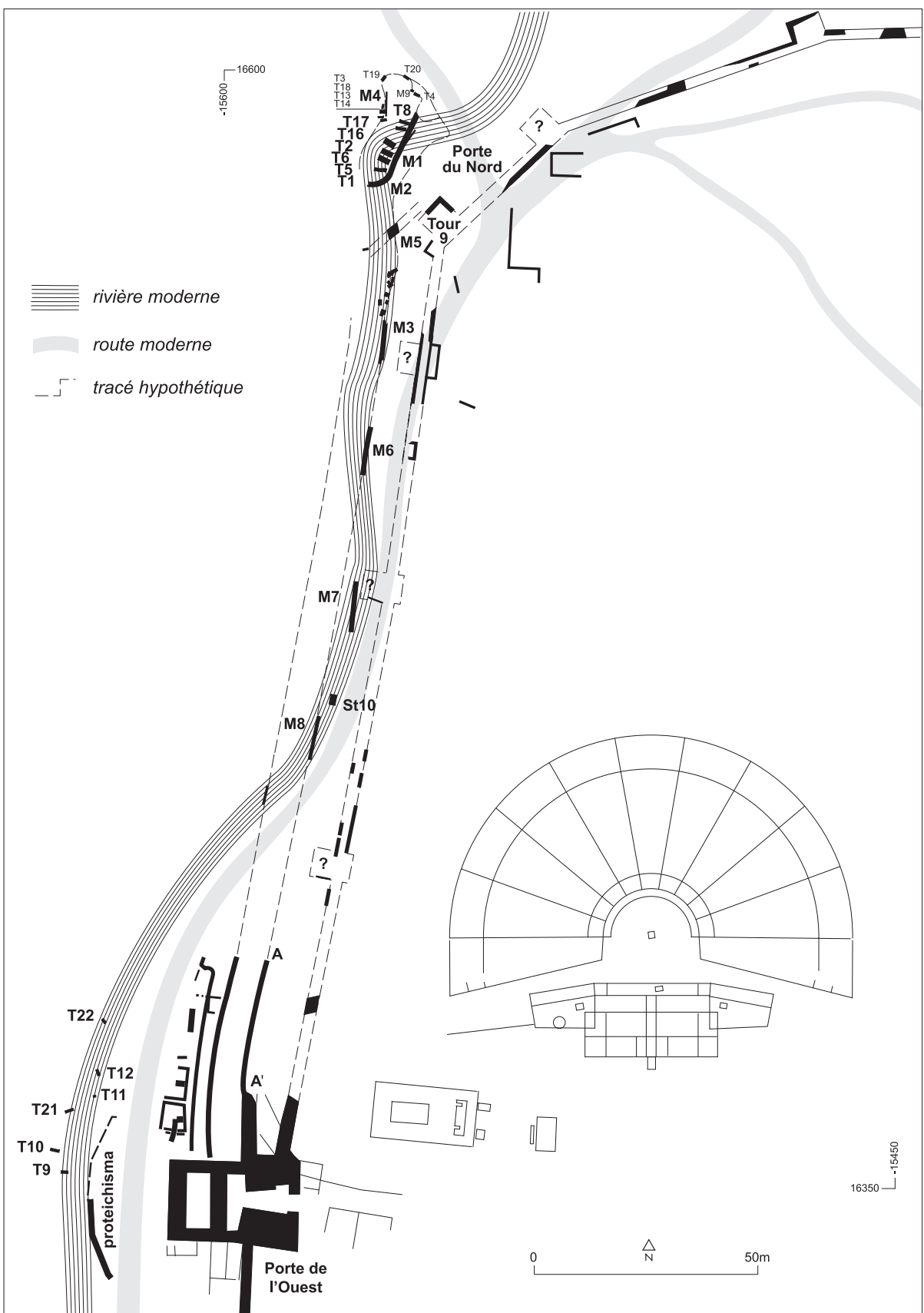

Fig. 4 - Plan du secteur Nord-Ouest de la ville d'Érétrie (ESAG, Th. Theurillat). 
massif H-I barrait désormais l'ancien bras oriental du cours d'eau et forçait les eaux à s'écouler en direction de la mer, canalisées par des murs de berge que l'on peut suivre par intermittence. Tant au Nord (sur 190 m) qu'au Sud (sur plus de 500 m) de la Porte, le cours d'eau saisonnier était désormais canalisé par deux murs rectilignes ${ }^{18}$.

En résumé, les découvertes réalisées en 2009 éclairent d'un jour nouveau l'importance de ce cours d'eau, qui marqua de son empreinte l'urbanisme érétrien à l'époque archaïque. Lampleur des travaux d'endiguement qui furent conduits à Érétrie à une époque aussi précoce est remarquable. En effet, ce n'est pas seulement le tronçon du cours d'eau se trouvant le plus proche du secteur de l'Héroon qui fut canalisé à cette date, mais l'ensemble de la berge orientale sur une longueur d'au moins $200 \mathrm{~m}$ en amont. Une telle entreprise, qui témoigne de la gestion consciente des risques naturels, ne trouve guère de parallèles dans la Grèce du viI ${ }^{e}$ s. Les travaux de 550 ne sont pas moins considérables, puisqu'ils aboutissent à remodeler le faciès géomorphologique du site et contribuent à assainir le développement urbanistique et défensif de la ville dans un environnement désormais stabilisé. Dès lors, le canal ne cessera de marquer la limite occidentale de la ville et de fonctionner comme fossé protecteur pour les murailles classique et hellénistique qui se succéderont sur ce front. Il établit également une ligne de démarcation entre le monde des vivants et celui des morts, puisque c'est à l'Ouest de celui-ci que va se développer la nécropole occidentale d'Érétrie.

\section{3. Le CANAL ET LE REMPART AUX ÉPOQUES ClASSIQUe ET HELLÉNiSTIQUE}

Les fouilles de 2009 n'ont fait qu'effleurer les murailles urbaines, mais elles permettent néanmoins de clarifier quelques problèmes topographiques et surtout d'ouvrir de

une fois de plus, que la Porte de l'Ouest présente plusieurs phases de construction documentées pour la période comprise entre 550 et 490 . Selon nous, son existence - associée aux vestiges de murs de fortification archaïques fouillés sur l'acropole - est une preuve solide de l'existence d'une muraille. Le cas de figure, proposé par certains, d'une porte «fortifiée» qui ne serait pas enceinte par des murailles mais laisserait la ville "ouverte», ne se justifie pas d'un point de vue poliorcétique. Nous sommes d'avis que le rempart archaïque, de part et d'autre de la Porte de l'Ouest, fut implanté en retrait du mur de berge pour le mettre à l'abri des crues, comme son successeur de la période classique. L'absence de vestiges s'expliquerait par le remploi des matériaux pour la construction de la muraille classique, un cas de figure aussi plausible que bien documenté ailleurs (R. FREDERIKSEN [n. 9], p. 41-49). Le cours d'eau, canalisé, est désormais un fossé, mettant ainsi les remparts à l'abri des travaux de sape et des machines de siège. Il ne fait aucun doute qu'il s'agit bien d'une ville pleinement fortifiée que les Perses assaillirent six jours durant en 490 (Hérodote, VI 101).

18. Ce mur de digue n'est pas attesté plus au Sud de B1000 et il est probable que le cours d'eau ne continuait pas tout droit vers le Sud à travers la nécropole archaïque, mais dans une baie/marécage au Nord-Ouest de l'Hygeionomeion. 
nouvelles pistes de recherche concernant le secteur Nord-Ouest de la ville. Mais avant de les énumérer, il n'est pas inutile de revenir brièvement sur la configuration des remparts classiques dans ce secteur.

Aux alentours de 400, soit une décennie après la libération de la cité du joug athénien, le principal effort financier des Érétriens fut consacré à la construction de nouvelles murailles urbaines protégeant l'ensemble du site. L'enceinte classique d'Érétrie atteignait une longueur de $4 \mathrm{~km}$ et comprenait une soixantaine de tours. C'est un projet édilitaire d'une portée considérable, qui n’a toujours pas été étudié à sa juste valeur ${ }^{19}$. La Porte de l'Ouest est alors renforcée et un nouveau pont enjambe le canal. De nouvelles courtines sont construites sur la terrasse retenue par le mur de digue oriental, récupérant selon toute vraisemblance le rempart archaïque. Les courtines, d'une largeur moyenne de 2,6 m et composées d'un socle de pierre en appareil polygonal surmonté d'une élévation de briques crues, sont défendues par des tours rectangulaires disposées tous les $50 \mathrm{~m}$ environ. Les tours sont disposées en saillie par rapport aux courtines, enjambant la terrasse et s'implantant parfois directement dans le canal. Toutefois, si le plan des murailles est fort bien connu au Sud de la Porte de l'Ouest, on ne peut en dire autant pour le secteur situé au Nord de celle-ci. Le tracé des courtines est des plus aléatoire, parfois tributaire d'anciens plans; de plus, aucune tour n'a été relevée entre la Porte et le secteur $\mathrm{C} / 300$. Or, comme on le verra, l'angle Nord-Ouest de la ville représente un point faible dans le dispositif défensif d'Érétrie, rendant sa compréhension encore plus souhaitable. Dans les lignes qui suivent, nous décrivons trois constructions qui nous paraissent liées, directement ou indirectement, au rempart, en partant de la Porte de l'Ouest jusqu'au carré $\mathrm{C} / 300$.

\section{Le proteichisma de la batterie de tir à la Porte de l'Ouest}

Une imposante structure composée de gros blocs de conglomérat fut à nouveau mise au jour en 2009 dans le lit du cours d'eau. Il s'agit d'un important ensemble architectural auquel se rattache le mur A-A’ repéré par C. Krause dans les années 1960. Cette construction s'est considérablement dégradée au cours des dernières décennies, comme l'indique la comparaison entre les vestiges relevés alors et son état actuel : une à deux assises de blocs de conglomérat ont disparu par endroits, sans doute arrachées par les fréquents travaux de curetage du canal réalisés au bulldozer. Le mur fait 1,20 m de large et son élévation devait atteindre au minimum 2,24 m dans l'Antiquité, mais certainement bien davantage.

19. Une monographie sur les murailles urbaines d'Érétrie est en préparation, sous la responsabilité de S. Fachard. 
Selon C. Krause ${ }^{20}$, cette construction serait un mur de soutènement marquant le déplacement du cours d'eau vers l'Ouest lors de la construction du «bastion» de la Porte de l'Ouest au début du $\mathrm{II}^{\mathrm{e}} \mathrm{s}$. (nous lui préférons le terme de "batterie/plateforme de tir» ou de "plate-forme d'artillerie» ${ }^{21}$ ). Ainsi déviées vers l'Ouest, les eaux saisonnières ne s'écouleraient donc pas sous le passage voûté de cette nouvelle batterie de tir (qui servirait de passage pour y effectuer des sorties) mais à l'Ouest du mur A-A'. Cette hypothèse a fait l'objet de plusieurs critiques ${ }^{22}$. Aujourd'hui, l'équation doit être reformulée en prenant en compte de nouveaux éléments.

En effet, six tombes d'époque classique marquant une zone d'ensevelissement furent découvertes en 2009 à l'Ouest du mur A-A', dans une berge moderne emportée par le déluge. L'implantation des tombes est à un niveau compris entre 8 et $9 \mathrm{~m}$, soit légèrement en dessous du niveau naturel repéré à 9-9,60 m entre le mur A-A' et la batterie de $\operatorname{tir}^{23}$. Le niveau d'implantation des tombes, recouvert par près de $2 \mathrm{~m}$ de sédiments, est très nettement visible dans la stratigraphie du canal. L'espace de 4,90 m compris entre les premières tombes et le mur A-A' laisserait, en théorie, un espace suffisant pour un cours d'eau ${ }^{24}$. Mais il faudrait alors reproduire un mur de soutènement pour retenir la nouvelle terrasse funéraire, dont on ne possède aucune trace tangible dans le lit actuel ${ }^{25}$. De surcroît, afin de confirmer l'hypothèse d'un cours d'eau à l'Ouest du mur A-A', il faudrait localiser les vestiges d'un mur barrant les flots du "vieux" canal au Nord de la Porte de l'Ouest. Or rien n'est visible non plus dans la longue stratigraphie du canal et dans le lit du cours d'eau moderne. Il paraît plus logique et raisonnable de restituer le tracé du cours d'eau sous le pont classique puis hellénistique de la Porte de l'Ouest.

La construction massive repérée en A-A', davantage qu'un mur de berge hellénistique, est en réalité un mur de fortification, un élément de défense avancé protégeant (et conçu parallèlement à) la batterie de tir de la Porte de l'Ouest. Ce proteichisma définit un espace abrité dans lequel des défenseurs pouvaient être rassemblés en vue d'une contre-attaque ou lors d'une sortie. De surcroît, ce mur avancé et profondément fondé empêchait les travaux de sape; il interdisait également aux tours mobiles de s'approcher de la

20. C. Krause (n. 12), p. 50-58.

21. Le second est dû à M.-Chr. Hellmann, L'architecture grecque 3. Habitat, urbanisme et fortifications (2010), p. 329. Sur ces questions de terminologie, voir S. Fachard (n. 8), p. 105 n. 99.

22. P. Ducrey, "La Porte de l'Ouest à Érétrie et les opérations navales romaines de 200-198 av. J.-C.», dans S. Buzzi et al. (éds), Zona archeologica: Festschrift für Hans Peter Isler zum 60. Geburtstag (2001), p. 115-126 et pl. 16-17; S. FaChard (n. 8), p. 106-107.

23. C. Krause (n. 12), p. 50 et n. 162.

24. Des tombes ont également pu exister au pied du mur A-A', emportées à l'époque moderne par le cours d'eau.

25. Il faut cependant relever un alignement probable de quelques moellons, mais son état de préservation est trop ruiné pour pouvoir en tirer quoi que ce soit. 
batterie de tir et aux béliers d'opérer. Ce proteichisma privilégie donc une défense active, parfaitement en accord avec les normes poliorcétiques de la fin du III et $^{\mathrm{e}}$ du début du II $^{\mathrm{e}} \mathrm{s}$. On retrouve de tels avant-murs, mutatis mutandis, à Syracuse et Sélinonte ${ }^{26}$. L'existence de ce proteichisma, confirme, nous semble-t-il, que la batterie de tir protégeant la Porte de l'Ouest est le fruit d'un plan élaboré, ambitieux et coûteux à mettre en place, et en aucun cas une construction réalisée dans l'urgence ${ }^{27}$.

\section{Le mur M6}

La crue de 2009 a révélé l'existence d'un mur polygonal inédit (M6) situé à $160 \mathrm{~m}$ de la Porte de l'Ouest. S'il est aligné sur l'axe du mur de digue oriental, M6 s'en distingue toutefois nettement par son mode d'appareillage : préservé sur une élévation de $1 \mathrm{~m}$, l'appareil, analogue à celui de la muraille classique, se compose de plusieurs assises de blocs polygonaux fort bien ajustés ${ }^{28}$ (fig. 4, 5). La longueur de M6 ne peut malheureusement pas être mesurée avec précision; un bloc disloqué dans la stratigraphie nous indique que le plan de cette construction pourrait se développer vers l'Est, même

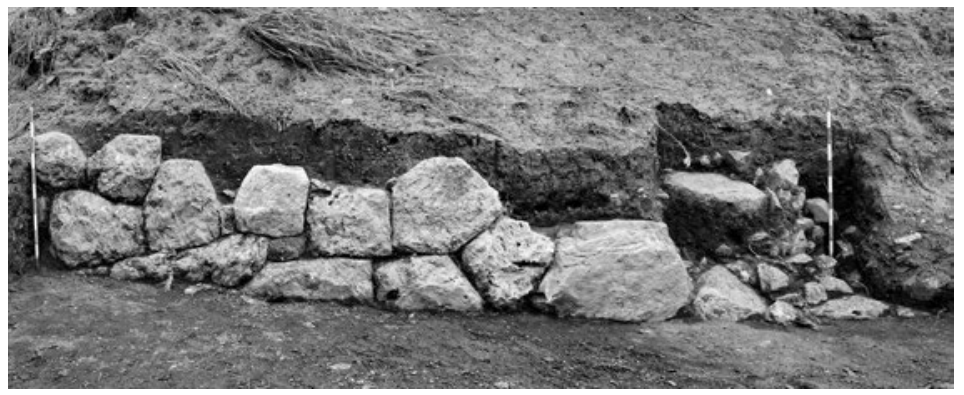

Fig. 5 - Le mur M6 vu depuis l'Ouest (cl. ESAG, S. Fachard).

26. A. W. Lawrence, Greek Aims in Fortification (1979), p. 288-289; J.-P. Adam, L'architecture militaire grecque (1982), p. 248-251; M.-Chr. Hellmann (n. 21), p. 334-235; H.-J. Beste, «The Castle Euryalos of Syracuse ", dans R. Frederiksen, S. Müth, P. I. Schneider, M. Schnelle (éds), Focus on Fortifications: New Research on Fortifications in the Ancient Mediterranean and the Near East, Fokus Fortifikation Studies 2 (2016), p. 193-206.

27. S. FACHARD (n. 8), p. 107.

28. Le calcaire employé provient des carrières de l'acropole. Les joints sont soignés, avec une anathyrose minimale sur les lits de pose et d'attente; la face des blocs est brute de carrière et ne comporte pas de bossage. La taille des blocs inférieurs atteint $82 \times 55 \mathrm{~cm}$ alors que celle des blocs des assises supérieures s'amenuise $(54 \times 45 ; 43 \times 45$, etc.), ce qui est courant dans les élévations. Le mur est légèrement biais, ce qui pourrait s'expliquer par le besoin de résister à une élévation. 
si aucun angle ni retour ne sont visibles ${ }^{29}$. À titre d'hypothèse, il nous semble probable de restituer une longueur comprise entre $9,50 \mathrm{~m}$ et $11 \mathrm{~m}$. Comme M6 n'est pas sur l'axe des remparts, mais sur celui du mur de berge oriental (A-M8-M3), il paraît à première vue logique de l'associer à un tronçon du mur de canalisation oriental. La différence dans l'appareil pourrait indiquer une réfection du mur de berge à l'époque classique. Mais cette explication se heurte à un argument, d'ordre essentiellement économique : pourquoi engager des tailleurs de pierre pour reconstruire une section de mur de berge dans un appareil polygonal soigné, alors qu'un assemblage de gros blocs lâchement appareillés aurait largement suffi? Ceci est d'autant plus surprenant qu'aucun autre segment du mur de canalisation ne présente un appareil aussi élaboré en dehors du secteur de la Porte de l'Ouest. Un travail ostentatoire pour la réfection d'un mur de berge ne se justifie pas a priori. En revanche, il trouverait davantage de sens s'il faisait partie de l'enceinte en appareil polygonal. Il serait certes trop avancé pour former une courtine, mais il pourrait, à titre d'hypothèse, appartenir au front oriental d'une tour. Toutefois, cette hypothèse se heurte à deux obstacles. En premier lieu, le rythme tour-courtine de l'enceinte classique à partir de la Porte de l'Ouest n'est pas connu avec précision, mais une projection basée sur les éléments à notre disposition suggère que l'emplacement de M6 ne saurait correspondre à celui d'une tour. Ensuite, la longueur de M6 (plus de 9 m) n'est pas en accord avec la largeur moyenne des tours du rempart classique (comprise entre 6,4 et $6,8 \mathrm{~m}^{30}$ ) et son avancée par rapport au rempart - dont le tracé n'est cependant pas bien connu dans ce secteur - paraît plus importante que la norme ${ }^{31}$. En l'état de nos connaissances, il nous semble plus raisonnable d'interpréter M6 comme un mur de berge classique. Ce dernier se situe à un endroit clef, là où le ruisseau initie son méandre vers l'Est. Il a sans doute été nécessaire de corriger le tracé du cours d'eau au IV ${ }^{\mathrm{e}}$ s. en forçant le courant à suivre un parcours rectiligne. L'appareil fruste des murs de berge archaïques n'était sans doute pas suffisamment solide pour contenir les flots, si bien qu'il fut décidé de construire un

29. La longueur du segment en appareil polygonal atteint $6,14 \mathrm{~m}$. Au Sud, on ne perçoit qu'une assise de blocs de fondation qui peut être partiellement suivie sur une longueur de $5 \mathrm{~m}$. Toutefois, seuls trois blocs sont contigus et présentent des lits d'attente suffisamment bien taillés pour recevoir une seconde assise de blocs polygonaux. Plus au Sud, les autres blocs affichent un alignement discontinu, ne présentent pas de signe de taille et le plus grand pourrait faire partie du rocher naturel.

30. Tout en notant que d'autres tours présentent une longueur plus importante : 9,6 m pour la tour Nord de l'acropole.

31. Comme la position des courtines n'est pas connue dans ce secteur, il est impossible de préciser la saillie de cette hypothétique tour. Toutefois, en prolongeant les courtines attestées au Sud et au Nord, cette dernière présenterait une saillie de plus de $8 \mathrm{~m}$ par rapport au rempart, alors que celle des autres tours classiques atteint souvent 4,9-5,5 m (mais 7,60 m pour la tour Nord de l'acropole). Il faudrait donc restituer une tour aux dimensions plus importantes que la norme, formant un élément de défense plus avancé, ou alors restituer les courtines plus à l'Ouest. Enfin, il faut relever que l'appareil de cette «nouvelle» tour se distingue de celui des tours T1, T2 et T3 visibles plus au Sud; en revanche, il s'apparente à celui des courtines de l'enceinte classique. 
nouveau mur de soutènement à cet endroit. Les tailleurs de pierre opérant sur la muraille furent sans doute responsables de la construction de cet ouvrage.

\section{Un pont sur le canal?}

Un autre mur polygonal inédit (M5) fut localisé à $210 \mathrm{~m}$ de la Porte de l'Ouest. Il s'agit d'un mur à double parement enfermant un remplissage interne, d'une largeur de 1,75 à 2,20 m, préservé sur une longueur de 3,31 m et orienté Nord-Est, Sud-Ouest ${ }^{32}$. Son tracé se poursuit sous la berge orientale, alors que son extension vers l'Ouest a été emportée par le courant. Ses fondations s'appuient partiellement sur un affleurement calcaire qui traverse le lit du cours d'eau et se poursuit vers le Sud-Ouest. L'élévation Nord est préservée sur une hauteur de $1 \mathrm{~m}$, alors qu'elle dépasse 1,40 m au Sud. Le mur M5 présente deux modes de construction distincts : à l'Est, un appareil polygonal fruste à bouchons et à empilage, avec des blocs de calcaire grossièrement ajustés entre eux, vraisemblablement sans avoir nécessité une taille des joints à pied d'œuvre (fig. 4, 6-7). Vers l'Ouest, où le mur s'interrompt dans le lit du ruisseau, le mur est repris en sousœuvre par des blocs de conglomérat, dont certains présentent des chanfreins. Quelques mètres au Sud, le lit du cours d'eau est encombré d'un amas de 12 blocs de conglomérat, dont plusieurs à chanfrein; selon toute vraisemblance, ils proviennent de M5, dont les

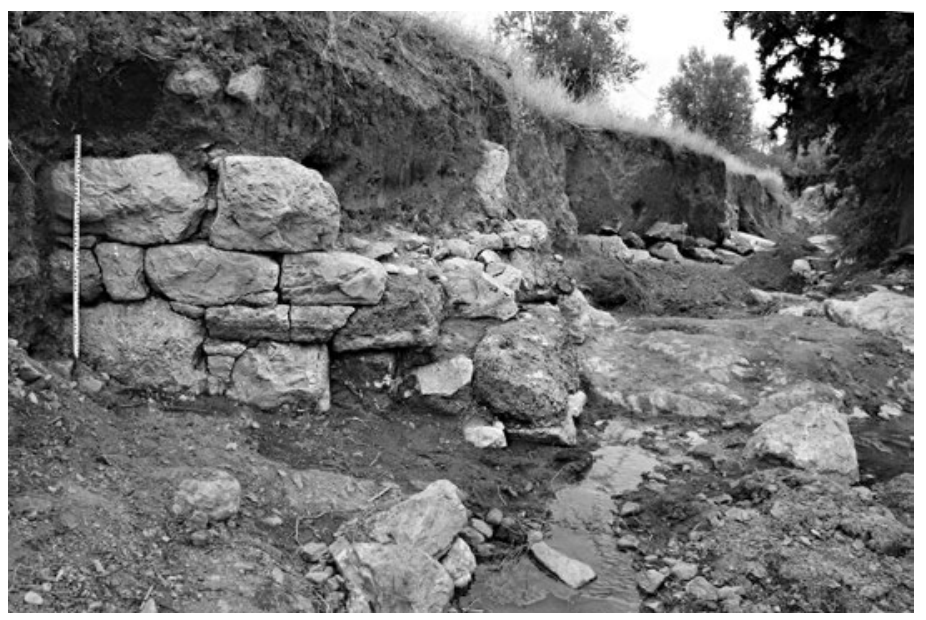

Fig. 6 - Le mur M5 vu depuis le Nord (cl. ESAG, S. Fachard).

32. Ce mur apparaît schématiquement sur plusieurs anciens plans, mais il n'a jamais été documenté ni photographié. 


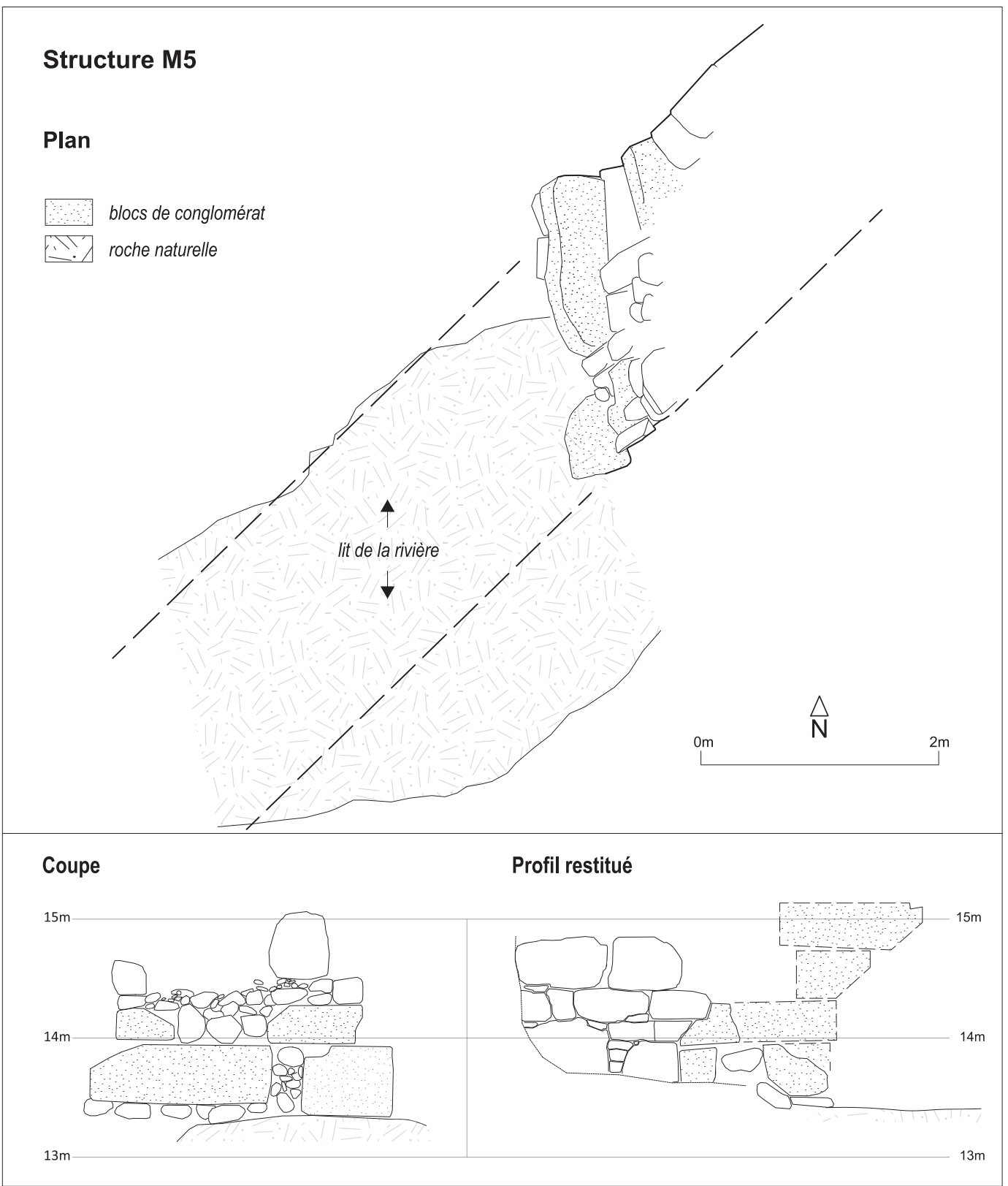

Fig. 7 - Plan, coupe et profil restitué de la structure M5 (ESAG, Th. Theurillat). 
composants se seraient effondrés dans le cours d'eau. Enfin, un amoncellement de blocs brisés et de pierres est visible dans l'alignement de M5 sur la berge opposée ${ }^{33}$. Soulignons encore que M5 fait face à la terrasse funéraire Nord (dont il sera question plus bas), délimitée par le mur M1 ; l'intervalle entre ces deux murs, d'une largeur d'environ $9 \mathrm{~m}$, est occupé par le cours d'eau antique. Enfin, M5 est situé à $2 \mathrm{~m}$ de la tour d'angle T9, dont les vestiges ont été découverts en $2013^{34}$.

Le plan et la fonction de M5 demeurent énigmatiques. Dans un premier temps, il nous a semblé que M5 pouvait faire partie d'un mur de berge supportant la tour T9. Un tel mur placerait la tour à l'abri des travaux de sape, mais surtout des courants ravageurs $\mathrm{du}$ cours d'eau saisonnier. Toutefois, cette hypothèse souffre de plusieurs faiblesses: pour pouvoir offrir une protection adéquate à la tour d'angle T9, il faudrait que M5 se prolonge d'une douzaine de mètres au Nord-Est, ce qui est loin d'être assuré; ensuite, si le but était de se prémunir contre les crues et les sapes, on ne comprend pas pourquoi il aurait été nécessaire de construire un mur polygonal à double parement, alors qu'un grossier mur de soutènement aurait suffi. Pour ces raisons, il nous paraît improbable que M5 puisse faire office de mur de berge ${ }^{35}$.

À l'évidence, le mur M5 fait partie d'une construction qui se prolongeait vers le SudOuest, donc dans le cours d'eau antique, et qui a été en partie emportée par le courant. Il ne peut s'agir d'un barrage, car M5 n'est pas suffisamment épais ni solide pour pouvoir contrer une crue et déplacer les eaux vers l'Ouest. Il faut plutôt restituer à cet endroit un pont à empilage qui enjambait le cours d'eau. M5 et les vestiges repérés dans le même axe sur la berge opposée doivent ainsi être interprétés comme les contreforts en grand appareil de l'ouvrage prenant appui sur les berges ${ }^{36}$, tandis que les blocs de conglomérat effondrés dans le lit du cours d'eau au Sud composent les éléments de culée et de voûte, supportant une passerelle en bois ou des dalles de pierre ${ }^{37}$ (fig. 8). Un tel pont a pu être réservé aux piétons et aux troupeaux, même si la largeur de M5 (1,75 m) autorisait en théorie le passage de chariots, dont l'écartement standard avoisinait 1,40-1,45 $\mathrm{m}^{38}$.

33. Cette partie de la berge faisant partie d'un terrain privé, il n'a pas été possible de les dégager.

34. Seuls quelques blocs sont visibles en plan, mais leur agencement ne laisse planer aucun doute. Il s'agit d'une tour carrée ou rectangulaire, disposée à l'angle Nord-Ouest de l'enceinte urbaine. L'emplacement théorique de la courtine suggère une saillie de $5,5 \mathrm{~m}$ par rapport à celle-ci.

35. Selon la configuration de ce secteur, nous pensons qu'un mur de berge devait se trouver quelques mètres plus au Nord.

36. Relevons que l'ouvrage n'est pas perpendiculaire au cours d'eau qu'il enjambe. Il s'agirait donc d'un pont biais.

37. Pour des ponts de ce type, voir M.-Chr. Hellmann (n. 21), p. 359.

38. Ibid., p. 357. 

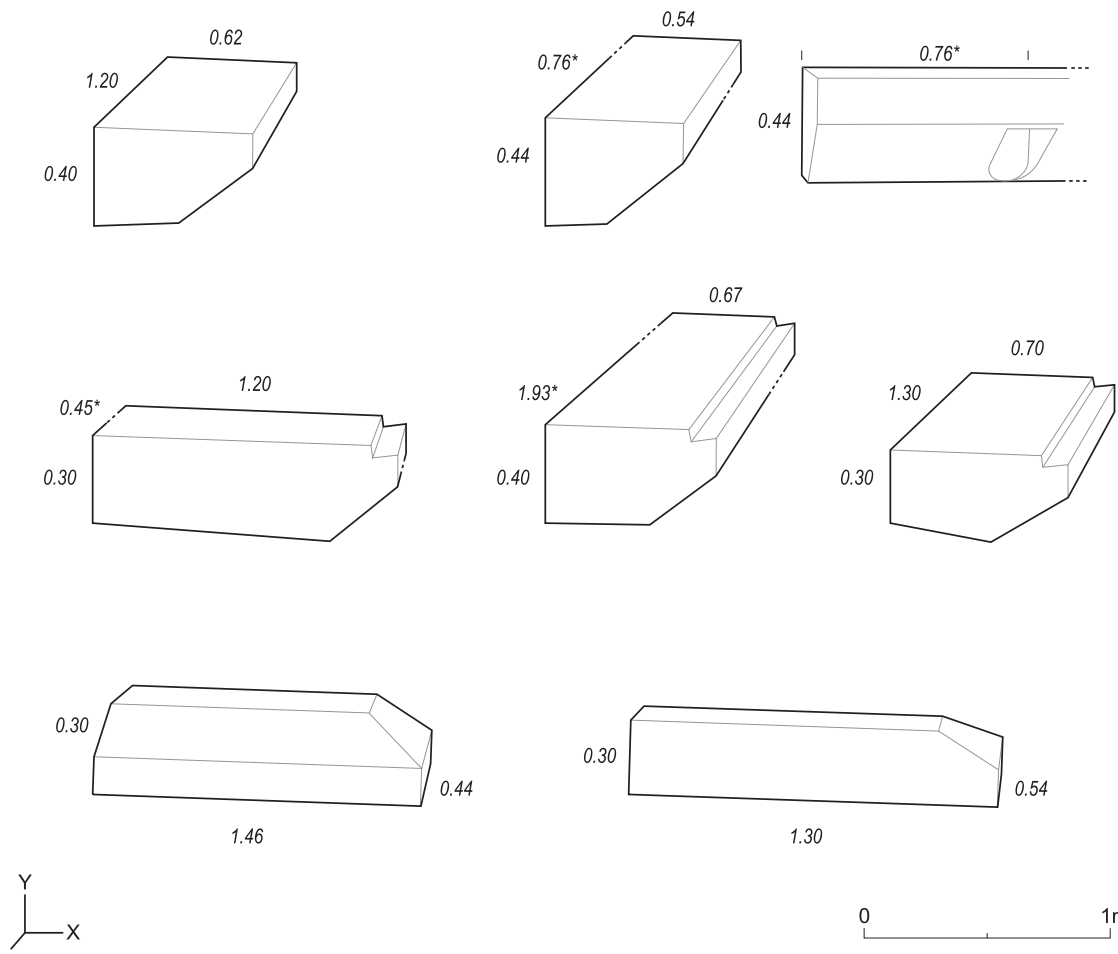

$1 \mathrm{~m}$

Fig. 8 - Éléments architecturaux appartenant probablement à un pont (M5) (ESAG, Th. Theurillat).

Deux éléments, et non des moindres, plaident en faveur de cette hypothèse. Premièrement, c'est dans ce secteur que les archéologues américains relevèrent à la fin $\mathrm{du} \mathrm{XIX}^{\mathrm{e}}$ s. un pont antique ("ancient Bridge»), reproduit tant sur la minute de travail que sur le plan final des remparts d'Érétrie (fig. 9). Il n’y a aucune raison valable de mettre en doute le témoignage de J. Pickard et J. W. Gilbert, qui passèrent les mois de janvier et mars 1891 à relever les vestiges du rempart et dont les observations se révèlent encore fort précieuses plus d'un siècle après leur publication ${ }^{39}$. Ensuite, les blocs à chanfrein emportés par le cours d'eau et effondrés au Sud de M5 correspondent bien à des éléments d'encorbellement. Le relevé des blocs permet de reconnaître trois types de

39. J. Pickard, "A Topographical Study of Eretria», AJA 1st Series 7 (1891), p. 371-389, pl. 19. 


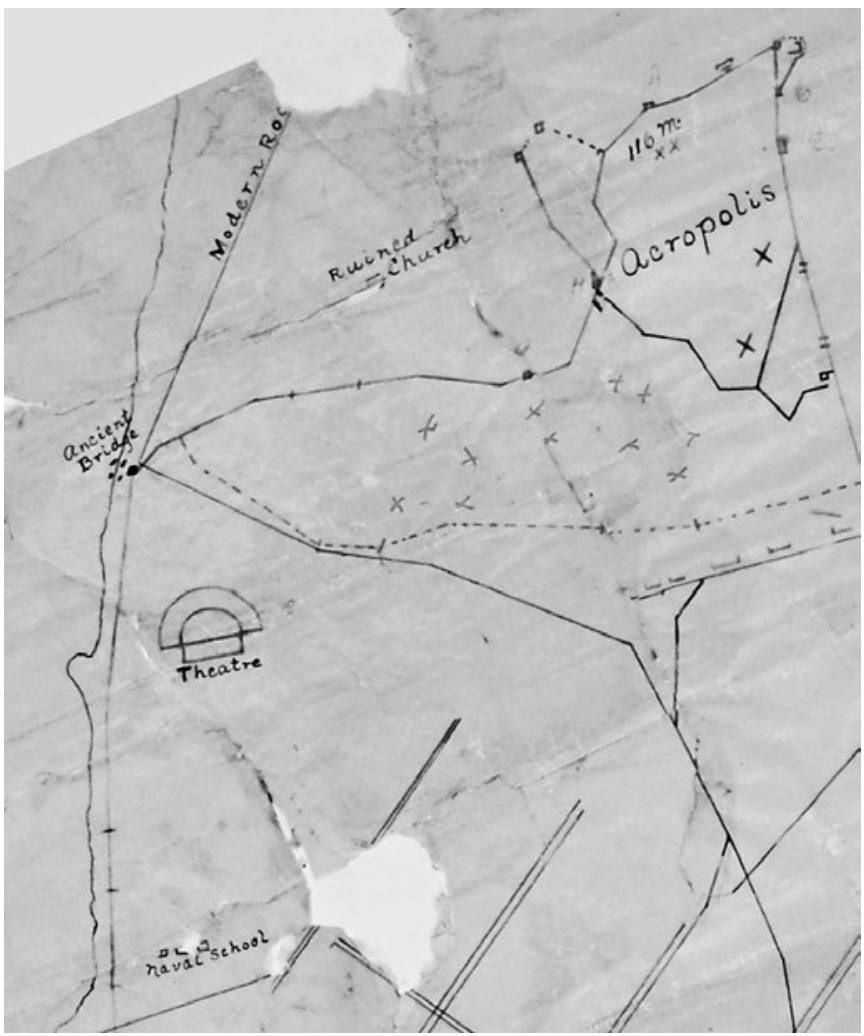

Fig. 9 - Reproduction de détail du plan d'Érétrie par J. Pickard et J. W. Gilbert (1891) (ASCSA, J. Pickard).

module, qui s'imbriquent parfaitement pour former un arc à degrés abattus ${ }^{40}$, tel qu’on le retrouve, toutes proportions gardées, dans le pont Nord d'Éleutherna ${ }^{41}$. Les éléments à notre disposition sont encore trop ténus pour pouvoir restituer le plan et l'élévation de ce pont en pierre, dont la date de construction, vraisemblablement durant l'époque classique-hellénistique, ne peut être précisée. Mais son existence est désormais assurée et sa localisation à l'angle Nord-Ouest de la ville s'accorde bien avec la présence d'une

40. R. GINouvìs, Dictionnaire méthodique de l'architecture grecque et romaine, tome II, Éléments constructifs : supports, couvertures, aménagements intérieurs (1992), p. 142 et pl. 70.3 d.

41. Sur ce pont, voir P. Bougia, Ancient Bridges in Greece and Coastal Asia Minor, Ph.D., University of Pennsylvania (1996), p. 267-271 et pl. 85-86; A. NAKAssis, "The Bridges of Ancient Eleutherna», ABSA 95 (2000), p. 353-365. 
porte à cet emplacement stratégique, qui devait s'ouvrir aux abords de la tour T9, qui la fortifiait au Sud.

\section{4. LA NÉCROPOLE OCCIDENTALE}

La façade occidentale du mur de fortification érétrien était percée de plusieurs portes au-delà desquelles se développaient plusieurs espaces funéraires. Au SudOuest, une porte mettait en communication le port et la nécropole archaïque de l'Hygeionomeion $^{42}$. À la Porte de l'Ouest, une série de tombeaux monumentaux fouillés à la fin des années 1960 reste en grande partie inédite ${ }^{43}$. Les vestiges mis au jour durant l'épisode torrentiel de 2009 permettent d'enrichir notre connaissance des lieux d'ensevelissement dans ce secteur ${ }^{44}$. La fouille fut conduite dans des conditions difficiles et les investigations se sont limitées au dégagement des boues torrentielles, révélant des contextes très perturbés. Un important matériel céramique provenant de tombes fut découvert hors de tout contexte (A6 et A7). Les sépultures étaient pour la plupart dans un état fragmentaire et ne contenaient que rarement des offrandes et des ossements. Toutes se trouvent sur la berge occidentale du cours d'eau actuel, ce qui fut vraisemblablement le cas dans l'Antiquité aussi : à l'Ouest du proteichisma, ce sont des tombes éparses apparues dans les berges ravinées, tandis qu’à proximité de la Porte du Nord, un enclos ou terrasse funéraire a pu être fouillé, qui faisait probablement partie d'une nécropole plus vaste (fig. Io). Un catalogue synthétique (table I) résume les principales caractéristiques des tombes qui ont pu être documentées, dont les lignes qui suivent présentent les traits les plus saillants ${ }^{45}$.

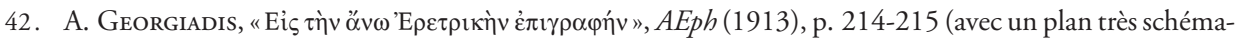
tique).

43. La fouille n'a fait l'objet que de brèves notices dans l'Archaiologikon Deltion. Voir P. Ducrey, S. Fachard, D. Knoepfler, Th. Theurillat, D. Wagner, A. Zannis (éds), Éétrie : guide de la cité antique (2004), p. 186-189 et en dernier lieu un mémoire de Master inédit, A.-L. Pradervand, Passer de vie à trépas à Érétrie. B/3 Nord dans tous ses états, Université de Lausanne (2015). Mentionnons encore une porte classique et hellénistique, quelque $200 \mathrm{~m}$ au Sud de la Porte de l'Ouest, visible aujourd'hui dans la cour du musée, qui commandait une route antique médiane, notée sur le plan américain de 1891 («Possible Sacred Road").

44. Pour un bilan sur les nécropoles érétriennes, voir en particulier K. GEx, Rotfgurige und weissgrundige Keramik, Eretria IX (1993), p. 11-24, ainsi que B. Blandin, Les pratiques funéraires d'époque géométrique à Éétrie. Espace des vivants, demeures des morts, Eretria XVII (2007), p. 31-37, et K. GEx, "Les rites et le mobilier funéraires du vi ${ }^{\mathrm{e}}$ au $\mathrm{II}^{\mathrm{e}}$ siècle av. J.-C.", dans C. Martin Pruvot et al. (éds), Cité sous terre : des archéologues suisses explorent la cité grecque d'Érétrie (2010), p. 267-270.

45. Certaines tombes (T4, T7, T13, T22) furent relevées et photographiées, sans pouvoir être précisément documentées. La publication exhaustive des tombes s'inscrit dans un futur projet d'étude des nécropoles érétriennes. 


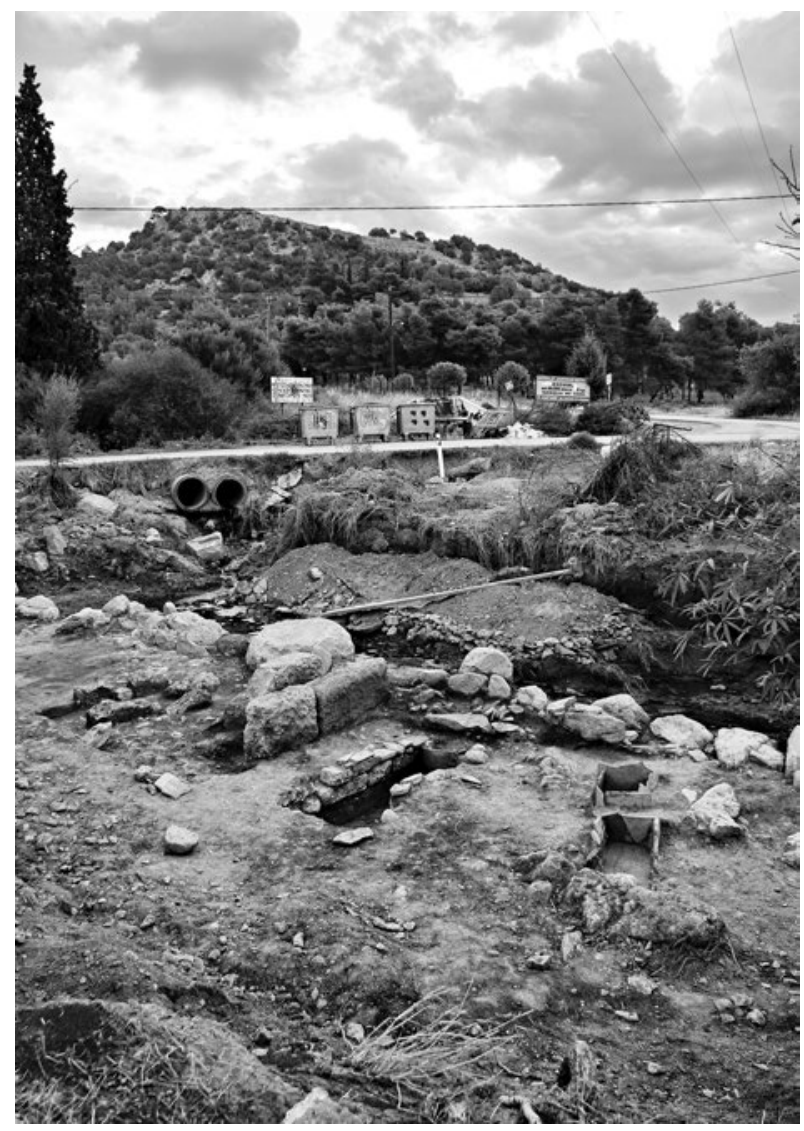

Fig. Io - Vue de la terrasse funéraire Nord. À l'arrière-plan, l'acropole d'Érétrie (cl. ESAG, Th. Theurillat).

\section{La terrasse funéraire Nord}

À l'angle Nord-Ouest de la ville, le cours d'eau saisonnier moderne marque un méandre très prononcé et le tracé de la fortification fait un coude en direction de l'acropole (on se situe là à quelque $230 \mathrm{~m}$ au Nord de la Porte de l'Ouest). C'est à cet endroit que s'est développé un espace funéraire, délimité à l'Est et au Sud par un long mur (M1), vraisemblablement composé de deux parements plusieurs fois consolidés et modifiés (fig. 4). M1 s'étend sur plus de $16 \mathrm{~m}$ selon une orientation Nord-Est/Sud-Ouest avant de marquer un virage vers l'Ouest. Au Nord, il disparait sous la route moderne. Il faisait vraisemblablement office de mur de terrasse bordant le lit du cours antique, ce dernier étant limité à l'Est par la berge (terrassée?) supportant la muraille urbaine. Le pont dont il 
a été question plus tôt se trouve à moins d'une dizaine de mètres au Sud-Est et permettait de traverser le cours d'eau pour entrer dans la ville par la Porte du Nord.

Le mur M1 retient une terrasse sur laquelle sont implantées sept tombes, relativement bien préservées: T1, T5, T6, T2, T16, T17 et T8. Les sépultures sont alignées perpendiculairement à l'axe formé par M1. On distingue trois inhumations (T6, T8 et T17) et deux incinérations probables (T2 et T16). Les dispositifs d'ensevelissement sont variés : tombes à ciste en terre cuite d'adultes (T8) et d'enfants (T1 et T5), tombe à ciste en conglomérat (T2), tombe à sarcophage en terre cuite (T17) et tombe à fosse maçonnée (T6 et T16). Cette diversité témoigne autant de la multiplicité des pratiques funéraires que de la longue durée d'utilisation de cette terrasse depuis le $\mathrm{v}^{\mathrm{e}}$ s. jusqu'au début de l'époque romaine, avec une apparente interruption à la basse époque hellénistique ${ }^{46}$.

La tombe T6 présente un dispositif complexe sur deux niveaux différents, indiquant deux phases d'utilisation distinctes : dans un premier temps, un défunt adulte fut inhumé dans un cercueil en bois, dont subsistent des clous en fer, disposé dans une fosse en pleine terre et scellé par des tuiles. Dans un second temps, les parois latérales de la fosse d'implantation au-dessus du cercueil furent revêtues d'une maçonnerie de tuiles et de pierres, dont un fragment d'inscription funéraire en remploi, daté de la seconde moitié du Iv ${ }^{e}$ s. (28). Ce second caveau ne contenait pas de vestiges osseux, mais a livré trois kotylés miniatures d'époque classique (A3-A5). On ignore s'il était destiné à recueillir un second défunt ou s'il constituait un aménagement en relation avec un culte funéraire rendu au premier défunt.

La tombe T17 appartient quant à elle à un mode d'ensevelissement rarement attesté à Érétrie : il s'agit d'un sarcophage en terre cuite formé de deux éléments. Le sarcophage, très délabré, a livré une unique offrande, à savoir un lécythe à fond blanc du dernier tiers du v $v^{\mathrm{e}}$. (A8) déposé au pied du défunt (fig. II-I2).

Un second mur de terrasse en retrait à l'Ouest (M4) délimite un autre espace funéraire comportant plusieurs tombes à tuiles et à ciste (T14, T13, T3, T18, T19), dont l'une datée de la fin du $\mathrm{IV}^{\mathrm{e}} \mathrm{s}$. (T3). Ces dernières, émergeant dans la stratigraphie de la berge effondrée, n'ont pu être que très partiellement documentées. Entre ces deux terrasses, un espace de 3 ou $4 \mathrm{~m}$ de large, que les circonstances n'ont pas permis de fouiller, a pu être occupé par une voie d'accès ou par une série de tombes. Enfin, deux sépultures furent découvertes dans le soutènement de la route moderne, entre M1 et M4 (T4, T20).

46. Voir infra, p. 165-171. 


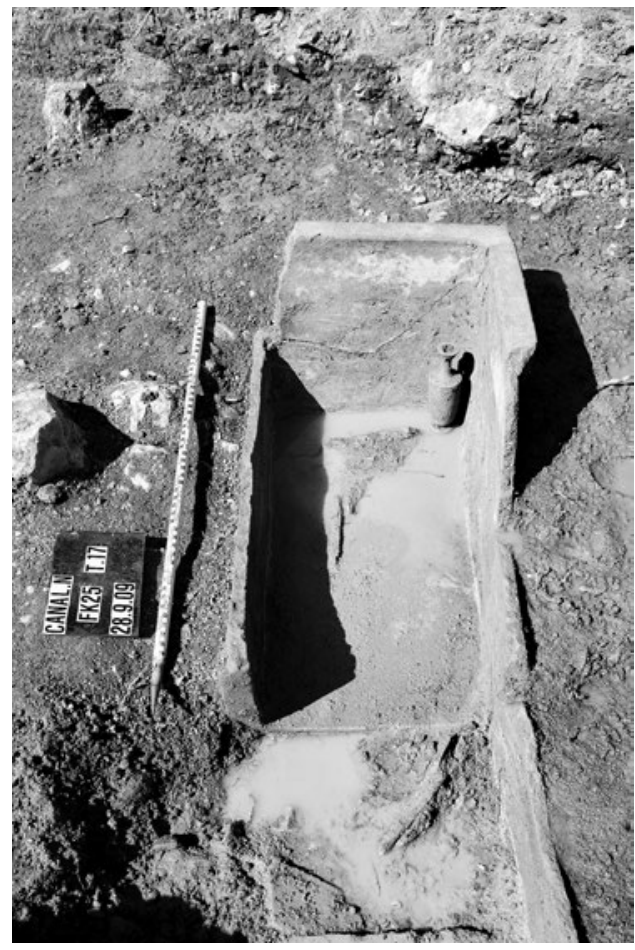

Fig. I I - Tombe T17 avec lécythe à fond blanc A8 en place lors de sa découverte (cl. ESAG, S. Fachard).

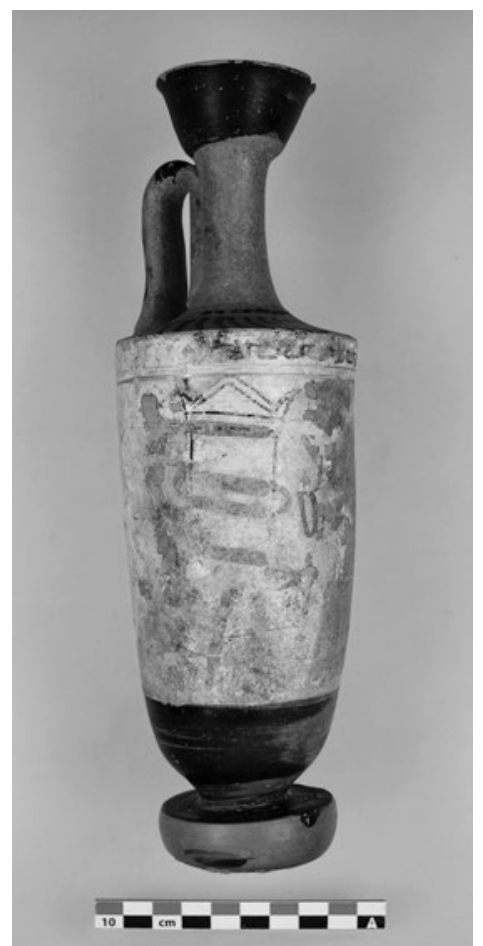

Fig. I2 - Lécythe à fond blanc A8 (cl. ESAG, S. Fachard).

\section{Le secteur du proteichisma}

Six autres tombes furent découvertes dans le lit du torrent et les berges au Nord-Ouest du proteichisma, la plupart très endommagées par l'inondation et sans matériel associé. Deux exceptions notables sont les tombes T9, qui a livré quelques beaux fragments de vases de la fin du v e s. (A9-A12), et T10, qui a conservé l'essentiel de ses offrandes (fig. I3). Le fond, les parois et la couverture de cette tombe à ciste sont faites de tuiles corinthiennes revêtues d'un enduit interne blanc-beige. Le crâne du défunt, dont les vestiges ont été emportés par les flots, était orienté vers l'Est. Le trousseau funéraire disposé le long du corps se composait d'une obole en bronze retrouvée à la hauteur des cervicales, d'un bolsal à anses horizontales (A19) et d'un bol simple à vernis noir (A23), de deux lécythes aryballisques miniatures à décor de fleuron (A21-22), d'une machaira ou kopis miniature en fer disposée à la hauteur de la main droite ${ }^{47}$,

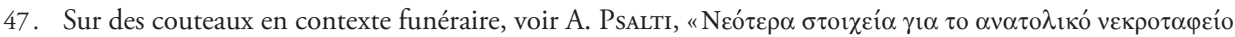

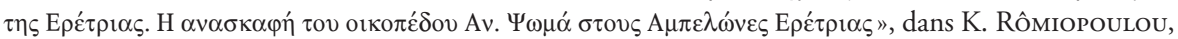




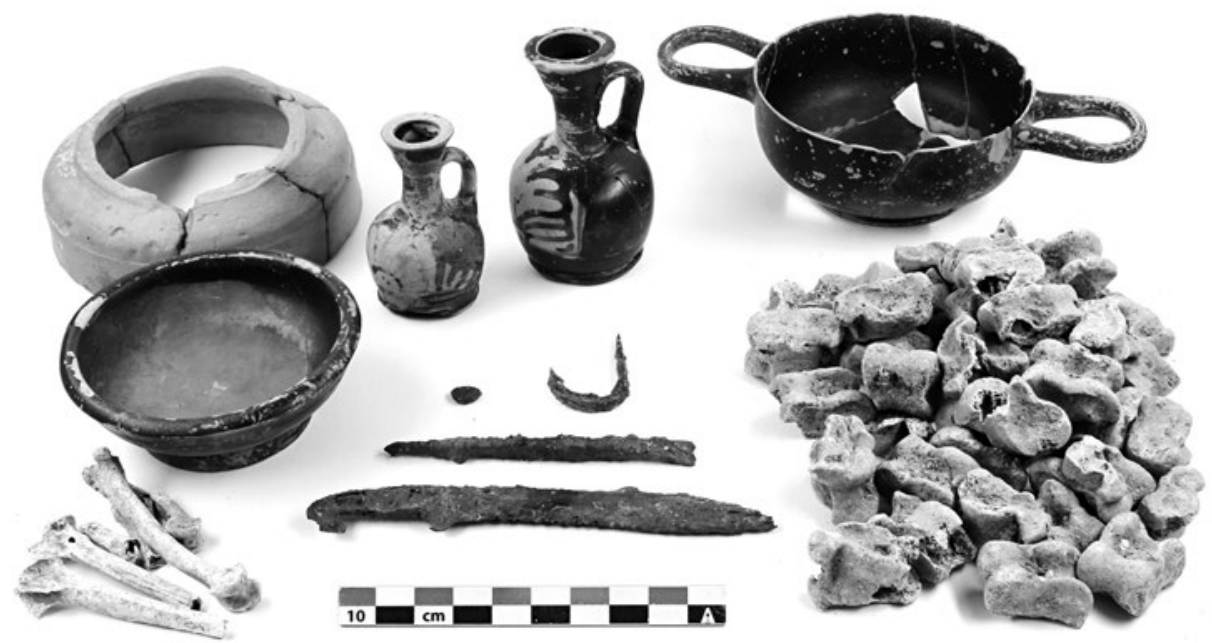

Fig. I3 - Offrandes découvertes dans la tombe T10 (cl. ESAG, T. Krapf).

d'un hameçon de pêche en bronze, d'une tige en fer indéterminée et de plusieurs dizaines d'osselets. Les ossements d'un petit oiseau (ou d'une volaille) furent déposés au niveau des jambes du défunt, probablement un enfant adolescent (taille estimée entre 1,40 et 1,45 cm). Le pied d'un lécythe géant a également été retrouvé lors du dégagement de la tombe (A20). Ce dernier ne faisait pas partie des offrandes, mais devait servir de marqueur de tombe ${ }^{48}$. C'est l'un des très rares cas où le contexte d'utilisation de cette production érétrienne est connue. La tombe $\mathrm{T} 10$ est datée de la fin du $\mathrm{v}^{\mathrm{e}}$ au début du $\mathrm{IV}^{\mathrm{e}}$ s. et est donc contemporaine des plus anciennes tombes de la nécropole $\mathrm{B} / 3$ Nord, une vingtaine de mètres à l'Est.

\section{La structure St10}

Située à une centaine de mètres au Nord de la Porte de l'Ouest, cette structure partiellement mise au jour prend la forme d'une base rectangulaire constituée de quatre blocs de conglomérat. Deux blocs disposés en orthostate forment le parement Ouest, contre lequel sont adossés deux blocs disposés à plat. Cette technique de construction

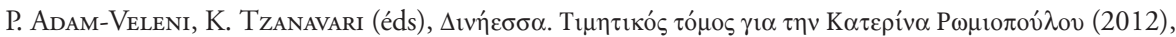

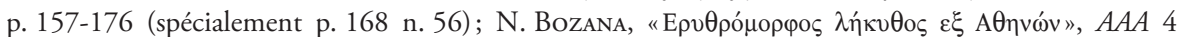
(1971), p. 255-261. Sur l'épée de type machaira, voir P. DuCrey, Guerre et guerriers dans la Grèce antique (1985), p. 93 ill. 61 et p. 96.

48. Sur ces lécythes géants, voir K. Gex, "Red-figure and White-ground Pottery from Euboean Workshops», dans S. Schierup, V. Sabetai (éds), The Regional Production of Red-figure Pottery: Greece, Magna Graecia and Etruria, Gösta Enbom Monographs 4 (2014), p. 121-135 (spécialement p. 127-129). 
et les matériaux mis en œuvre sont similaires à ceux des monuments funéraires en $B / 3$ Nord. S'agit-il également ici des fondations d'un tombeau? Plusieurs observations rendent cette hypothèse fragile, sans pouvoir toutefois l'infirmer totalement. En premier lieu, l'altitude de la structure paraît trop basse pour pouvoir reposer sur la terrasse du rempart ${ }^{49}$. Ensuite, les tombeaux et sépultures connus, au nombre d'une trentaine à l'heure actuelle, sont systématiquement placés sur la berge occidentale du canal et jamais sur la berge du rempart, qui semble avoir été volontairement laissée libre de constructions ${ }^{50}$ et dont l'usage devait être strictement réservé aux opérations de défense et de l'entretien de la muraille ${ }^{51}$.

\section{APPENDICES}

\section{Catalogue céramique (A. Psalti)}

A1 Canal Nord, tombe T5 (FK 3), ME 21493. Askos globulaire. Pied annulaire, cordon à la transition entre l'épaule et le col (ce dernier étant partiellement préservé), anse incomplète. Vernis noir mat partiellement écaillé sur le pied et surface inférieure réservée. Pâte beigebrune. Diam. pied 3,4 cm; haut. 4,7 cm. Cf. Agora XXIX 1141. Fin IV'-début III ${ }^{\mathrm{e}} \mathrm{s}$.

A2 Canal Nord, tombe T3 (FK 20), ME 21494. Olpé complète. Panse ovoïde. Pied annulaire. Vernis brun opaque, écaillé. Pâte beige-orange. Atelier local. Haut. 12,5 cm; diam. base 4,5 cm; diam. col 2,6 cm. Cf. Eretria VI, p. 82, pl. 42, 3. Fin IV s.

A3 Canal Nord, tombe T6 (FK 7), ME 21495. Kotylé partiellement préservée. Départ d'une anse horizontale, panse conique, lèvre légèrement évasée vers l'extérieur, pied conique. Vernis noir sur la surface interne, le pied et la partie inférieure de la panse vernissés; une bande divise la

49. L'altitude de la terrasse du rempart n'est certes pas connue avec précision, mais l'altitude supérieure du mur de berge M3 suggère un niveau situé à au moins 13,09 m. Or ici, les blocs de conglomérat se trouvent à une altitude de 11,44 m, soit 1,65 $\mathrm{m}$ en dessous du niveau théorique minimal de circulation sur la terrasse du rempart. L'implantation de fondations aussi profondes, creusées entre le mur de berge et le rempart, pour supporter un tombeau se justifie difficilement.

50. À notre connaissance, la seule structure recensée est le "puits» archaïque découvert à la hauteur de la tour T1 du Quartier de l'Ouest, dont l'interprétation est complexe, voir P. Auberson, «À propos d'un puits public à Érétrie», $B C H 99$ (1975), p. 789-799; C. KRAUSE, "Brunnentürme in der archaischen Stadtmauer Eretrias», $A K 25$ (1982), p. 39-43; M. CATTIN, «Le puits dans la muraille archaïque du Quartier de l'Ouest", mémoire de Master inédit, Université de Neuchâtel (2008). Toutefois, cette construction n'était plus en place lors de la construction du rempart classique.

51. En effet, un enchevêtrement de tombeaux et de monuments divers dans cette zone, certains pouvant atteindre 1 à $2 \mathrm{~m}$ d'élévation, aurait en réalité uniquement servi la cause d'un assaillant, lui offrant des caches contre les tirs des défenseurs, des plateformes de tir surélevées et surtout des estrades sur lesquelles placer des échelles. 
panse en deux parties, partie supérieure réservée. Pâte orange-beige clair. Diam. pied : 6,2 cm; diam. 6,5 cm; haut. 4,6 cm. Cf. Eretria VII, p. 64, pl. 15, 218. ve-IV s.

A4 Canal Nord, tombe T6 (FK 7), ME 21496. Kotylé miniature (intacte à l'exception d'une anse horizontale). Panse et pieds coniques. Anse horizontale à ruban. Vernis négligé à l'intérieur. La panse n'est pas vernissée à l'exception d'une bande horizontale brune en son centre. Des petits traits verticaux sommaires recouvrent la jonction entre la panse et l'anse. Pâte orange-beige. Diam. pied $3 \mathrm{~cm}$; diam. $5,5 \mathrm{~cm}$; haut. 4,1 cm. Cf. Eretria VII, p. 64, pl. 15, 219. $v^{\mathrm{e}}-\mathrm{IV}$ e $s$.

A5 Canal Nord, tombe T6 (FK 7), ME 21497. Partie inférieure d'une kotylé miniature. Vernis dilué à l'intérieur, absent à l'extérieur. Traces d'une bande décorative sur la partie basse de la panse. Pied plat et peu élevé. Diam. $2,9 \mathrm{~cm}$; haut. $2,4 \mathrm{~cm}$.

A6 Canal, nettoyage de surface (FK vrac), ME 21499 (V 4911). Lécythe aryballisque. Panse cylindrique, lèvre et anse incomplètes. Vernis noir externe, à l'exception de trois bandes réservées sur la panse. Pâte beige-orange. Diam. pied 3,9 cm; diam. col 2,7 cm; haut. 8,7 cm. Cf. Psalti (2012) (n. 47), p. 160, fig. 6, nº AE 18618. Production attique. Début du Iv s.

A7 Canal Sud, secteur du proteichisma (FK vrac), ME 21498 (V 4910). Fig. 14. Lécythe aryballisque. Intact à l'exception d'un fragment de la lèvre et d'un léger impact sur l'épaule. Décor en technique de la figure rouge représentant une figure féminine ailée (Sirène) se tenant sur une branche de laurier. Vernis noir mat. Pâte orange-beige. Diam. pied 4,3 cm; diam. col 2,7 cm; haut. 9,5 cm. Production attique. Milieu du ve s. Attribué au Seireniske Painter. Cf. J. D. Beazley, Attic Red-Figure Vase-Painters' ${ }^{2}$ (1963) [1942], vol. 1 et 2 , p. $487,704, \mathrm{n}^{\text {os }} 56,68$.

A8 Canal Nord, tombe T17 (FK 25), ME 20167 (V 4912). Fig. 12. Lécythe à fond blanc («type secondaire»). Catégorie a ATL (D. C. KurTz, Athenian White Lekythoi [1975], p. 82-83, pl. 21-23). Pâte brune

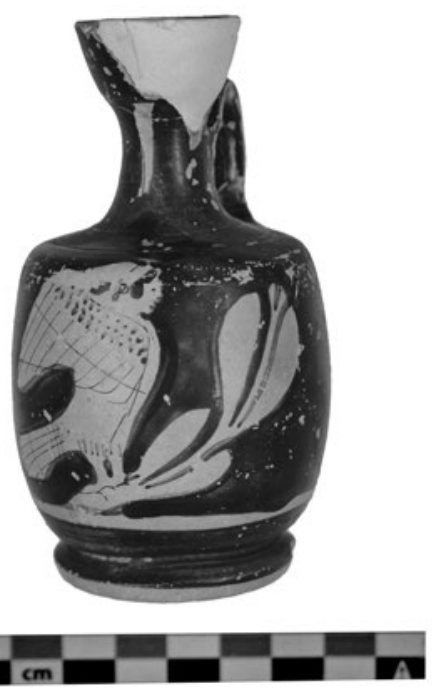

Fig. 14 - Lécythe aryballisque A7 (cl. ESAG, S. Fachard). claire. Le fond blanc et la scène peinte sont en grande partie écaillés. Vernis noir sur l'embouchure. Anse verticale aplanie recouverte de vernis noir sur sa face externe. Sur l'épaule, décor quelque peu négligé, composé de points et traits. Le décor principal, peint sur fond blanc, se déploie sur la panse. Son 
thème consiste en une visite au tombeau. Dans le cadre supérieur, délimité par deux bandes jaunes, un décor de méandre qui penche vers la droite. Une scène de visite au tombeau est représentée dans le panneau central. On aperçoit, au centre, une stèle à fronton et acrotères enveloppée d'un bandeau rouge; à droite, une figure masculine (des boucles de cheveux hélicoïdales sont préservées) drapée d'un manteau dépose une couronne rouge sur la tombe. Une couronne de fruits rouges et noirs est suspendue. En raison de son mauvais état de préservation, une attribution à un peintre est impossible. La représentation de la stèle offre des parallèles : cf. D. C. Kurtz, op. cit., pl. 49,1 et 50,1 (dernier quart du ve s.), en provenance d'Érétrie. Diam. pied $5 \mathrm{~cm}$; diam. bord $5 \mathrm{~cm}$; haut. $23 \mathrm{~cm}$. La présence de lécythes à fond blanc dans les tombes érétriennes est particulièrement fréquente dans le troisième tiers du v ve Cf. Ch. Martin Pruvot et al. (n. 44), p. 302-303, nos 305-308; D. C. Kurtz, op. cit., p. 137-138; Musée du Louvre, CA 537 (vers 425).

A9 Canal Sud, secteur du proteichisma, tombe T9 (FK 6), ME 21500 (V 4908). Fig. 15. Fragment de lébès gamikos. Décor en technique de la figure rouge représentant une figure féminine revêtue d'un manteau, représentée de face avec la tête tournée vers la gauche. Elle tient dans sa main droite un alabastre, et dans sa main gauche un objet non identifié. Une figure ailée est représentée dans la partie gauche du fragment préservé. Un fleuron se trouve entre les deux figures. Pâte orange. Kymation ionique dans la partie inférieure de la scène. Dimensions du fragment : $13 \times 9,8 \mathrm{~cm}$. Deux fragments appartiennent vraisemblablement à la même pièce: fragment de figure féminine tenant dans sa main droite un kalathos. Cf. Agora XXX 151 et 141. Production attique. Dernier tiers du $\mathrm{v}^{\mathrm{e}} \mathrm{s}$.

A10 Canal Sud, secteur du proteichisma, tombe T9 (FK 6), ME 21502 (V 4907). Fragment de lécythe aryballisque. Décor en technique de la figure rouge représentant une sphinge assise. Pâte beige-orange. Dimensions du fragment : 6,2 × 3,6 cm. Cf. Agora XXX 1165. Production attique. Fin $\mathrm{v}^{\mathrm{e}} \mathrm{s}$.

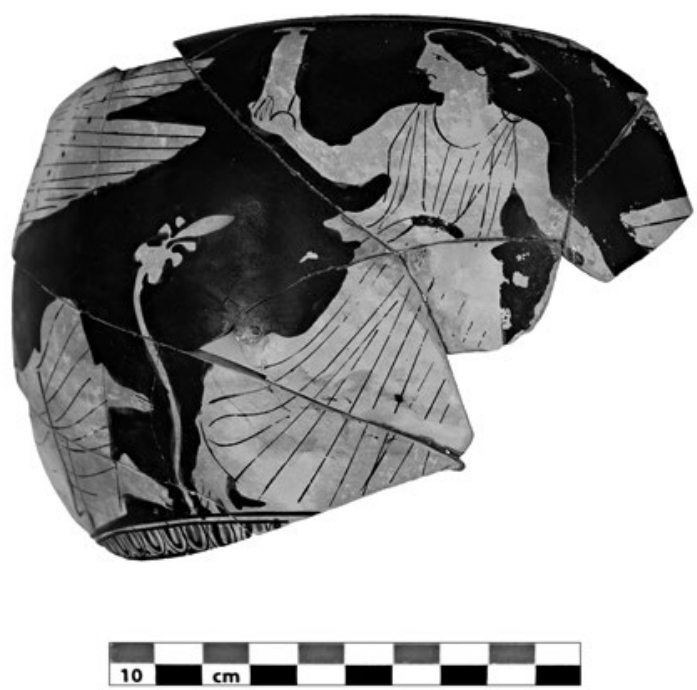

Fig. I5 - Fragment de lébès gamikos A9 (cl. ESAG, S. Fachard).

A11 Canal Sud, secteur du proteichisma, tombe T9 (FK 6), ME 21503 (V 4909). Fragment d'embouchure de lécythe. Vernis noir. Pâte orange-beige. Diam. lèvre $4 \mathrm{~cm}$; haut. cons. $3 \mathrm{~cm}$. 
A12 Canal Sud, secteur du proteichisma, tombe T9 (FK 6), ME 21504. Pied de vase fermé. Vernis noir et ocre sur la surface inférieure du pied. Pâte orange-beige. Diam. pied $6,5 \mathrm{~cm}$; haut. cons. $1,5 \mathrm{~cm}$.

A13 Canal Nord, tombe T16 (FK 24), ME 21505. Fig. 16. Unguentarium bulbeux. Pâte brune

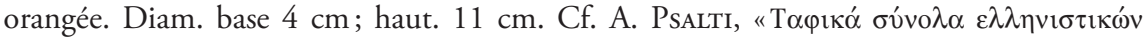

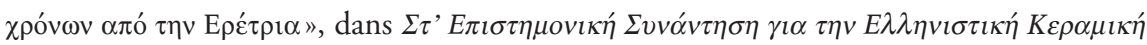
(2004), p. 505-512. $\mathrm{I}^{\mathrm{er}}$ s. av.- $\mathrm{I}^{\mathrm{er}}$ s. apr. J.-C.

A14 Canal Nord, tombe T16 (FK 24), ME 21506 (V 4906). Fig. 16. Unguentarium bulbeux. Panse particulièrement gonflée, base plate, col cylindrique allongé, lèvre en forme d'anneau. Pâte brune orangée. Diam. base $4 \mathrm{~cm}$; diam. bord $3,4 \mathrm{~cm}$; haut. $13 \mathrm{~cm}$. Cf. A. Psalti, loc. cit. supra (A13). $\mathrm{I}^{\mathrm{er}}$ s. av.-- $\mathrm{I}^{\mathrm{er}}$ s. apr. J.-C.

A15 Canal Nord, tombe T16 (FK 24), ME 21507. Fig. 16. Unguentarium bulbeux. Base plate, col cylindrique allongé, lèvre en forme d'anneau. Pâte brune orangée. Diam. base $3 \mathrm{~cm}$; diam. bord $3 \mathrm{~cm}$; haut. $12 \mathrm{~cm}$. Cf. A. Psalti, loc. cit. supra (A13). $\mathrm{I}^{\mathrm{er}}$ s. av.- ${ }^{\mathrm{er}}$ s. apr. J.-C.

A16 Canal Nord, tombe T16 (FK 24), ME 21508 (V 4905). Fig. 16. Unguentarium bulbeux. Base plate, col cylindrique allongé, lèvre en forme d'anneau. Pâte brune orangée. Diam. base $2,5 \mathrm{~cm}$; diam. bord 2,5 cm; haut. $11 \mathrm{~cm}$. Cf. A. Psalti, loc. cit. supra (A13). $\mathrm{I}^{\mathrm{er}}$ s. av.-I $\mathrm{I}^{\mathrm{er}}$ s. apr. J.-C.

A17 Canal Nord, tombe T16 (FK 24), ME 21509 (V 4905). Fig. 16. Unguentarium bulbeux. Base plate, col cylindrique allongé, lèvre en forme d'anneau. Pâte brune orangée. Diam. base $4 \mathrm{~cm}$; diam. bord $3 \mathrm{~cm}$; haut. $11 \mathrm{~cm}$. Cf. A. Psalti, loc. cit. supra (A13). $\mathrm{I}^{\mathrm{er}}$ s. av.- $\mathrm{I}^{\mathrm{er}} \mathrm{s}$. apr. J.-C.

A18 Canal Nord, tombe T16 (FK 24), ME 21510. Fragment d'unguentarium bulbeux. Col et fragment de panse préservés; lèvre évasée vers l'extérieur. Pâte brune foncée. Diam. bord $2,5 \mathrm{~cm}$; haut. 8,5 cm. Cf. A. Psalti, loc. cit. supra (A13). $\mathrm{I}^{\mathrm{er}}$ s. av.- $\mathrm{I}^{\mathrm{er}}$ s. apr. J.-C.

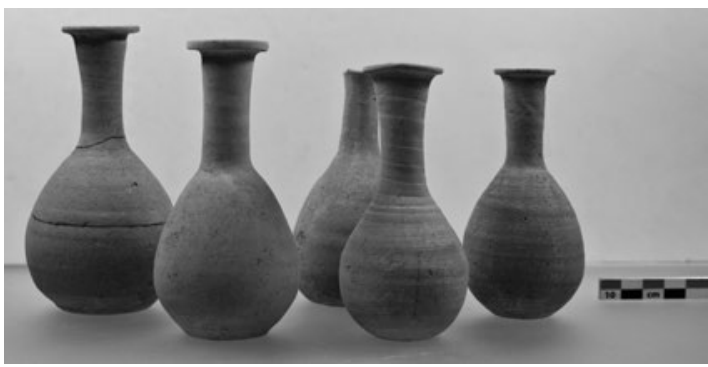

Fig. I6 - Unguentaria bulbeux A13-A17 (cl. ESAG, S. Fachard).
A19 Canal Sud, secteur du diateichisma, tombe T10 (FK 9), ME 21511 (V 4903). Bolsal. Anses horizontales et pied annulaire. Vernis noir lustré, à l'exception de la surface inférieure du pied qui est réservée avec un décor de deux cercles concentriques et d'un point central. Pâte brune foncée. Cf. Agora XII 542 et 556. Production attique. Dernier quart du $\mathrm{v}^{\mathrm{e}} \mathrm{s}$. 
A20 Canal Sud, secteur du proteichisma, tombe T10 (FK 9), ME 21512 (V 4900). Pied de lécythe géant. Pâte orange-brune. Diam. pied 10,5 cm; haut. cons. $4 \mathrm{~cm}$. L'absence de fond suggère que ce vase était utilisé pour faire des libations. De tels lécythes en provenance d'Érétrie ont été identifiés, de par leur taille importante, à des marqueurs de tombe. Cf. M. Golubic, Les lécythes à fond blanc géants de la nécropole du B/3 Nord à Érétrie. Mémoire de licence inédit, Université de Fribourg (1999), nº 24, pl. 24 (provenance B3/ ord, FK 1800). Fin du ve-début du $\mathrm{IV}^{\mathrm{e}} \mathrm{s}$.

A21 Canal Sud, secteur du proteichisma, tombe T10 (FK 9-3), ME 21513 (V 4904). Lécythe aryballisque miniature. Col évasé. Vernis noir inégalement réparti. Motif de fleuron. Pâte orange-brune. Diam. pied 3,5 cm; diam. bord 2,8 cm; haut. $8 \mathrm{~cm}$. Cf. Agora XII 11391140 ; Eretria IX, p. 61-62. Production attique. $\mathrm{IV}^{\mathrm{e}} \mathrm{s}$.

A22 Canal Sud, secteur du proteichisma, tombe T10 (FK 9), ME 21514 (V 4901). Lécythe aryballisque miniature. Pied concave. Vernis noir clairsemé. Décor négligé de fleuron. Pâte brune claire. Cf. Agora XII 1139-1140; Eretria IX, p. 61-62. Production attique. IV s.

A23 Canal Sud, secteur du proteichisma, tombe T10 (FK 9), ME 21515 (V 4902). Bol. Lèvre simple, sommet rond, base large. Vernis noir externe et noir-ocre partiel à l'intérieur. Pâte brune claire. Diam. pied $5 \mathrm{~cm}$; diam. bord 8,5 cm; haut. 3,5 cm. Cf. Agora XII 882. Fin ve-début Iv s.

\begin{tabular}{|c|c|c|c|c|c|}
\hline $\mathrm{N}^{\circ}$ & Type & Dimensions & Description sommaire & Mobilier & $\begin{array}{l}\text { Datation } \\
\text { de la céramique }\end{array}$ \\
\hline $\mathrm{T} 1$ & $\begin{array}{l}\text { Tombe à ciste } \\
\text { (enfant?) }\end{array}$ & $78 \times 66 \mathrm{~cm}$ & $\begin{array}{l}\text { Parois formées de quatre tuiles de type laco- } \\
\text { nien; une cinquième tuile est posée à plat } \\
\text { pour le fond. }\end{array}$ & $\begin{array}{l}\text { FK } 2 \\
\text { Skyphos à vernis } \\
\text { noir }\end{array}$ & Classique? \\
\hline $\mathrm{T} 2$ & Tombe à ciste & $129 \times 213 \mathrm{~cm}^{*}$ & $\begin{array}{l}\text { Parois formées par des blocs de conglomérat. } \\
\text { Ossements très fragmentaires (certains } \\
\text { sont calcinés) trouvés sous une couche de } \\
\text { démolition. }\end{array}$ & FK 15 & $?$ \\
\hline T3 & $\begin{array}{l}\text { Tombe sous } \\
\text { tuiles }\end{array}$ & $\begin{array}{l}\text { Largeur: } \\
\text { env. } 66 \mathrm{~cm}\end{array}$ & $\begin{array}{l}\text { Seul le fond de la tombe a été localisé dans } \\
\text { la paroi de la berge. Quelques os résiduels. }\end{array}$ & $\begin{array}{l}\text { FK } 20 \\
\text { A2 }\end{array}$ & Fin $\mathrm{IV}^{\mathrm{e}} \mathrm{s}$. \\
\hline T5 & Tombe à ciste & $72 \times 138^{*} \mathrm{~cm}$ & $\begin{array}{l}\text { La tombe s'appuie contre M1. Les parois } \\
\text { restantes sont composées de six tuiles } \\
\text { corinthiennes; deux tuiles sont placées à plat } \\
\text { au fond de la tombe. }\end{array}$ & $\begin{array}{l}\text { FK } 3 \\
\text { A1 }\end{array}$ & Fin $\mathrm{IV}^{\mathrm{e}}$-début $\mathrm{III}^{\mathrm{e}} \mathrm{s}$. \\
\hline T6 & $\begin{array}{l}\text { Tombe collec- } \\
\text { tive à fosse ma- } \\
\text { çonnée }\end{array}$ & $200 \times 94 \mathrm{~cm}$ & $\begin{array}{l}\text { Les parois latérales sont revêtues d'une } \\
\text { maçonnerie de pierres et de tuiles, dont un } \\
\text { fragment d'inscription ( } \mathbf{2 8} \text { ). Une tuile posée } \\
\text { de chant marque une séparation verticale. Au } \\
\text { niveau inférieur, séparé par des tuiles posées } \\
\text { à plat, un probable cercueil de bois ( } 3 \text { clous } \\
\text { préservés) renfermait la partie basse d'un } \\
\text { squelette orienté vers l'Est. }\end{array}$ & $\begin{array}{l}\text { FK } 7,12,13,16, \\
22 \\
\text { A3 } \\
\text { A4 } \\
\text { A5 } \\
3 \text { clous en fer }\end{array}$ & $V^{e}-I V^{e} s$ \\
\hline
\end{tabular}




\begin{tabular}{|c|c|c|c|c|c|}
\hline T8 & Tombe à ciste & $\begin{array}{l}213 \times 40-58 \\
\mathrm{~cm}\end{array}$ & $\begin{array}{l}\text { Parois formées par huit tuiles corinthiennes } \\
(68,5 \times 49 \times 3,5-5,7 \mathrm{~cm}) \text {; trois tuiles sont } \\
\text { posées à plat. Découverte de deux os longs } \\
\text { dans la boue. }\end{array}$ & $\begin{array}{l}\text { FK 4, 5, } 26 \text { Tes- } \\
\text { sons de céramique } \\
\text { fine et } 2 \text { clous en } \\
\text { fer }\end{array}$ & Classique? \\
\hline T9 & Tombe à ciste & $62 \times 53 \mathrm{~cm}$ & $\begin{array}{l}\text { Parois composées de tuiles. La tombe est } \\
\text { partiellement visible en coupe, dans la berge. }\end{array}$ & $\begin{array}{l}\text { FK 6 } \\
\text { A9 } \\
\text { A10 } \\
\text { A11 } \\
\text { A12 } \\
\text { Fragments de } \\
\text { bronze }\end{array}$ & $\begin{array}{l}\text { Dernier tiers du } \\
v^{e} s . \\
\text { Fin } v^{e} s \text {. }\end{array}$ \\
\hline $\mathrm{T} 10$ & Tombe à ciste & $57 \times 151 \mathrm{~cm}$ & $\begin{array}{l}\text { Parois composées de tuiles corinthiennes } \\
(50 \times 66 \mathrm{~cm}) \text {. Squelette très mal conservé, } \\
\text { position dorsale, orienté tête à l'Est. Les } \\
\text { parois sont recouvertes d'un enduit blanc- } \\
\text { beige. }\end{array}$ & \begin{tabular}{|l} 
FK 9 \\
1 obole \\
A19 \\
A20, A23 \\
\\
A21, A22 \\
- machaira \\
miniature en fer \\
- hameçon en \\
bronze \\
- osselets \\
- os d'un petit \\
volatile \\
- tige en fer indét. \\
(broche?)
\end{tabular} & $\begin{array}{l}425-400 \text {. } \\
\text { Fin } V^{\mathrm{e}} \text {-début } \mathrm{IV}^{\mathrm{e}} \mathrm{s} \text {. } \\
\mathrm{IV}^{\mathrm{e}} \mathrm{s} \text {. }\end{array}$ \\
\hline T11 & Tombe à ciste? & $42^{*} \times 60^{*} \mathrm{~cm}$ & $\begin{array}{l}\text { Couverture de tuile fragmentaire, quelques } \\
\text { os appartenant aux pieds du défunt. }\end{array}$ & $\begin{array}{l}\text { FK } 10 \\
\text { Pas de matériel }\end{array}$ & \\
\hline T12 & $\begin{array}{l}\text { Tombe sous } \\
\text { tuiles }\end{array}$ & $140^{*} \times 57^{*} \mathrm{~cm}$ & $\begin{array}{l}\text { Tombe composée d'une simple fosse cou- } \\
\text { verte de tuiles convexes à vernis jaune. } \\
\text { L'humérus et une partie du crâne et de la } \\
\text { mâchoire sont préservés; tête orientée à l'Est. }\end{array}$ & $\begin{array}{l}\text { FK } 11 \\
\text { Pas de matériel }\end{array}$ & Archaïque? \\
\hline T14 & Tombe à ciste & $51^{*} \times 50^{*} \mathrm{~cm}$ & $\begin{array}{l}\text { Tombe très perturbée dont il ne subsiste que } \\
\text { le fond (tuile posée à plat). }\end{array}$ & $\begin{array}{l}\text { FK } 19 \\
\text { Pas de matériel }\end{array}$ & \\
\hline T15 & $\begin{array}{l}\text { Tombe sous } \\
\text { tuiles }\end{array}$ & $65 \times 90^{*} \mathrm{~cm}$ & Squelette mal conservé, orienté tête à l'Est. & $\begin{array}{l}\text { FK } 21 \\
\text { Pas de matériel }\end{array}$ & \\
\hline T16 & $\begin{array}{l}\text { Tombe à fosse } \\
\text { maçonnée }\end{array}$ & $114 \times 160^{*} \mathrm{~cm}$ & $\begin{array}{l}\text { Parois revêtues de fragments de blocs de } \\
\text { conglomérat, appareil à blocs et empilage. }\end{array}$ & $\begin{array}{l}\text { FK } 24 \\
\text { A13, A14, A15, } \\
\text { A16, A17, A18 }\end{array}$ & $\left\{\begin{array}{l}\mathrm{I}^{\mathrm{er}} s \cdot \text { av. }-\mathrm{I}^{\mathrm{er}} \mathrm{s} . \\
\text { apr. J.-C. }\end{array}\right.$ \\
\hline T17 & $\begin{array}{l}\text { Tombe à } \\
\text { sarcophage }\end{array}$ & $\begin{array}{l}200 \times 50 \times \\
46,5 \mathrm{~cm}\end{array}$ & $\begin{array}{l}\text { Sarcophage de terre cuite, composé de deux } \\
\text { pièces. Pieds et fragment d'un fémur préser- } \\
\text { vés; squelette orienté tête à l'Est. }\end{array}$ & $\begin{array}{l}\text { FK } 25 \\
\text { A8 }\end{array}$ & $350-300$. \\
\hline $\mathrm{T} 21$ & Tombe à ciste & $69 \times 55^{*} \mathrm{~cm}$ & Parois et fond composés de tuiles. & - & Classique? \\
\hline
\end{tabular}

* dimensions approximatives en raison de l'état fragmentaire de la tombe

Table I — Sépultures du canal. 


\section{LES STĖLES FUNÉRAIRES (D. Ackermann, D. Knoepfler) ${ }^{52}$}

\section{iI. 1. Présentation du corpus (D. Ackermann)}

Les 29 stèles funéraires présentées ici ont été trouvées en position secondaire, éparpillées dans le lit du torrent. Elles ont été révélées par la crue de 2009, mais elles avaient sans doute été délogées de leur emplacement originel déjà dans l'Antiquité : la nécropole a dû en effet subir des destructions à l'époque hellénistique, notamment en 198, lorsque les Romains ont fait le siège de la ville occupée par les Macédoniens ${ }^{53}$. On ne s'étonnera donc pas qu'aucune de nos stèles n'ait pu être mise en relation avec l'une des tombes retrouvées.

Elles constituent un ensemble remarquablement varié, tant pour l'aspect des monuments et les matériaux utilisés que pour la formulation des épitaphes elles-mêmes. La chronologie est en revanche assez homogène : toutes paraissent dater entre l'extrême fin du $v^{e}$ et la seconde moitié du $\operatorname{III}^{\mathrm{e}} \mathrm{s} .{ }^{54}$. On retrouvait déjà cette même fourchette chronologique pour les stèles funéraires des alentours de la Porte de l'Ouest éditées en 1978 par Christiane Dunant ${ }^{55}$. Toutes les formes attestées dans notre échantillonnage peuvent être rattachées à la typologie élaborée par cette dernière, typologie à laquelle nous nous sommes référés. Les quelques variantes constatées, comme l'absence d'une ciselure périmétrique ou l'aménagement de la face antérieure jusqu’à la base, sont signalées, mais elles résultent de choix techniques de la part du tailleur de pierre et ne constituent en aucun cas des critères typologiques. Par ailleurs, l'ensemble publié en 1978 ne comportait aucune stèle à relief figuré, alors que nous en avons ici un exemplaire $(\mathbf{1})^{56}$. Pour le reste, la plupart des stèles sont conventionnelles : légèrement pyramidantes, l'arrière laissé brut ou piqué (font exception les numéros $\mathbf{1 1}$ et $\mathbf{2 3}$ ), les côtés travaillés au ciseau grain d'orge et pourvus d'une ciselure. Le mode opératoire de l'aménagement de la face antérieure des stèles est assez semblable d'un exemplaire à l'autre : dégrossissage à la broche (une partie est parfois laissée ainsi à la base de la stèle), taille intermédiaire au ciseau grain d'orge et surfaçage à l'aide d'une ripe à dents. Une bonne partie des stèles ne dépasse cependant pas le stade du dégrossissage : seul un bandeau est aménagé au ciseau grain d'orge ou plus fréquemment au ciseau, pour recevoir l'inscription. Une bande lisse est assez souvent

52. D. Ackermann prépare une monographie sur les monuments funéraires inscrits d'Érétrie, en collaboration avec D. Knoepfler. Elle tient à exprimer sa gratitude à la Gerda Henkel Stiftung et à la Kommission für Alte Geschichte und Epigraphik, et en particulier à son directeur Chr. Schuler, pour lui avoir permis d'effectuer un séjour de recherche à Munich en lien avec le présent article.

53. Voir infra, p. 204, et la conclusion p. 223.

54. Voir aussi les considérations chronologiques présentées par D. Knoepfler infra, p. 198-208.

55. Chr. Dunant, «Stèles funéraires», dans Eretria VI (1978), p. 21-61.

56. Sur l’absence dans notre lot de naïskos et de stèle à anthémion, voir infra, p. 200-202. 
aménagée au ciseau sur le pourtour de la stèle; cette ciselure périmétrique mesure en moyenne $1 \mathrm{~cm}$ de largeur. Les moulures le cas échéant, sont réalisées au ciseau ${ }^{57}$.

Pour ce qui est des matériaux utilisés, toutes les stèles ont été taillées dans du marbre ou du calcaire de provenance locale. Seule la stèle 11 a été taillée dans une plaque de micaschiste, qui provient probablement du Sud de l'Eubée ${ }^{58}$. Les concrétions sont abondantes sur les marbres et calcaires, ce qui rend parfois l'examen de la pierre un peu difficile.

Les datations proposées sont fondées sur la paléographie, la linguistique, la formulation des épitaphes, ainsi que sur la typologie des stèles; pour l'exemplaire 1, le style et l'iconographie du relief figuré ont aussi été pris en compte. Les datations étant assez larges et parfois incertaines, nous avons préféré classer les objets en fonction des informations livrées par l'épitaphe : d'abord les noms seuls, puis les noms et patronymes, enfin les noms et ethniques. À l'intérieur de chaque rubrique, nous avons suivi l'ordre alphabétique des noms des défunts. La stèle $\mathbf{1}$ fait exception : il s'agit de la seule stèle de l'ensemble à être ornée d'un relief figuré, c'est pourquoi nous l'avons placée à part, en première position.

Les numéros d'inventaire sont ceux du Musée d'Érétrie, abrégé ME. Les mesures sont données en centimètres, dans l'ordre hauteur-largeur-épaisseur. Sauf mention contraire, la hauteur indiquée est la hauteur maximale. Les mesures pour la largeur et l'épaisseur sont données de haut en bas.

\section{1) Thettalè. Thettalè fille de Nikôn (fig. 17)}

ME 20064.

Marbre gris-bleu.

60,5 × 37-39 × 7,6-7. Stèle pyramidante; épaisseur remarquablement régulière.

Stèle à sommet triangulaire, complète. Abîmée aux angles supérieurs gauche et droit par deux gros éclats. Quelques éclats sur les bords. Face antérieure ripée à la ripe à dents, sauf l'extrémité inférieure, travaillée à la gradine. Sur les côtés, ciselure et gradine. L'arrière est très grossièrement piqué.

Type Dunant II A.

Dans la partie triangulaire de la stèle, un triangle est incisé très finement, représentant un fronton. En dessous, à $1,5 \mathrm{~cm}$, est incisée une fine ligne droite sur toute la largeur, représentant une corniche. En dessous se trouve l'inscription, et en dessous encore, un relief dans un rectangle creux.

57. Par «ciseau» nous entendons le ciseau gravelet, à distinguer du ciseau grain d'orge. Pour l'analyse des traces d'outils sur les stèles, nous avons bénéficié de l'expertise de Thierry Grégor, que nous remercions chaleureusement.

58. Nos plus vifs remerciements à Pierre Gex pour ses observations sur la pétrographie des divers supports. 
Pour l'analyse iconographique et stylistique du relief, voir infra, p. 196-198.

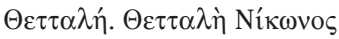

Linscription est gravée juste au-dessus du relief. Elle n'est pas centrée (marge à gauche : 2,7 ; à droite : 1,2 ).

Lettres petites, un peu épaisses, régulières, assez soignées. Hauteur des lettres régulière : premier nom : 1,2 (thêta: 1,1); second nom: 1,2-1,4 (thêta : 1,1; omicron : 0,9; oméga : 0,7). Espacement : 0,5-0,8 (premier nom); 0,2-0,4 (second nom, sauf thêta-epsilon, omicronsigma: 0,6, et nu-omicron: 0,5). Les lettres du second nom sont plus serrées et débordent sur la marge à droite, car il est plus long en raison de la présence du patronyme.

Forme des lettres: épaississements, lettres rondes plus petites (sauf le thêta, ou à peine), alpha à barre droite, haste horizontale médiane de l'epsilon plus courte, thêta avec un point à l'intérieur, premier $n u$ équilibré mais second $n u$ déséquilibré, sigma aux branches écartées, oméga en arche de pont.

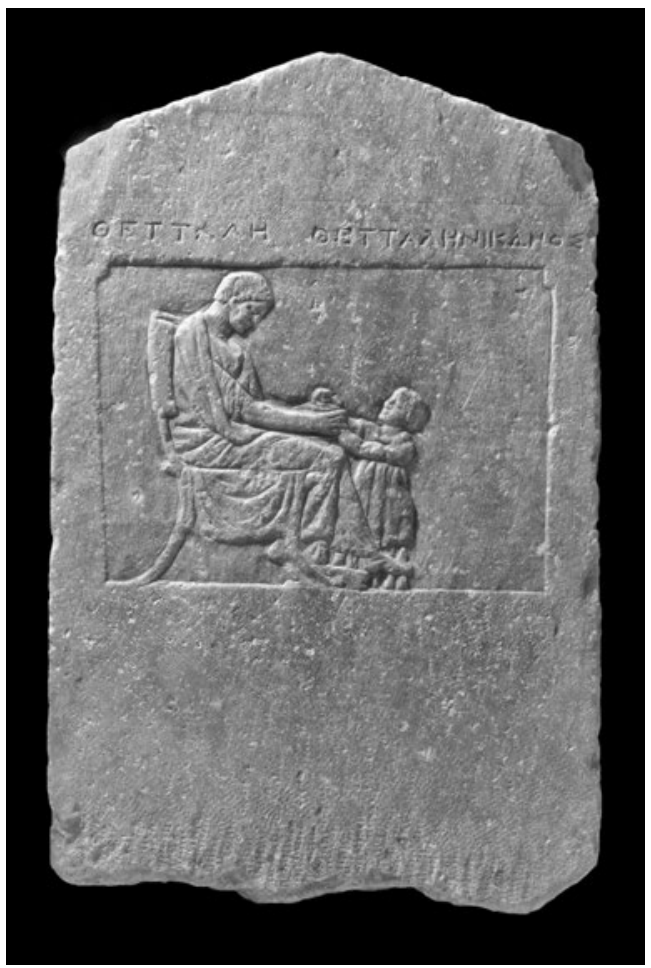

Fig. 17 — Stèle 1 (cl. ESAG, A. Skiadaressis). $3^{\text {e }}$ quart du Iv ${ }^{\mathrm{e}} \mathrm{s}$.

Les deux noms ont été gravés par la même main. Les défuntes portent le même onoma; il doit s'agir d'une mère (le premier nom, gravé au-dessus de la femme assise) et de sa fille (le second nom, gravé au-dessus de l'enfant).

\section{2) Hapalè (fig. 18)}

ME 20046.

Marbre gris-bleu.

$58 \times 29-31 \times 7,5-10,6$. Stèle pyramidante, plus épaisse en bas.

Stèle à sommet rectiligne, complète sauf l'angle supérieur gauche, amputé. Éclats sur la face antérieure dans les bords, dont un gros en haut à droite qui a abîmé la dernière lettre, sans gêner la lecture pour autant. Concrétions abondantes sur toute la face antérieure de la stèle, un peu moins cependant dans la partie inférieure gauche. Face antérieure dégrossie à la broche, bandeau lisse $(3,5 \mathrm{~cm})$ pour l'inscription, réalisé au ciseau. Pas de ciselure périmétrique. Les côtés sont brochés. L'arrière est très grossièrement piqué. 
Type Dunant I B.

A $\pi \alpha \dot{\alpha} \lambda \eta$

L'inscription est gravée à $8 \mathrm{~cm}$ du sommet de la stèle. Elle n'est pas parfaitement centrée (marge à gauche : 1,7 ; à droite : 2,4). Lettres fines, régulières, soignées. Hauteur des lettres parfaitement régulière : 3. Espacement : 2,8 (alpha-pi, pi-alpha), 2,4 (alphalambda), 2,6 (lambda-êta).

Forme des lettres: alpha à barre droite, $p i$ à haste verticale droite s'interrompant à mi-hauteur.

$1^{\text {re }}$ moitié du IV $s$.

\section{3) Aristarchos (fig. 19)}

ME 20084.

Marbre gris-bleu.

56,2 (avec le couronnement) $\times 27-30,5 \times 8,8$. Stèle pyramidante; épaisseur remarquablement régulière.

Le couronnement est formé d'un bandeau saillant $(2,8$ $\mathrm{cm})$ et d'un chanfrein droit $(2 \mathrm{~cm})$. Décrochement du couronnement par rapport au fût de la stèle : 1,6.

"Stèle rectangulaire à moulure en saillie» selon la typologie de Chr. Dunant (cf. son $\mathrm{n}^{\circ}$ 60), brisée en bas, en haut à gauche du couronnement et dans l'angle supérieur droit du couronnement. Éclats dans les bords (sur le couronnement et sur le reste de la stèle), gros éclat en haut à droite qui a fait disparaître la moitié inférieure du sigma final. Concrétions abondantes en haut et en bas de la stèle, beaucoup plus réduites en son centre. Face antérieure ripée à la ripe à dents. Sur les côtés, ciselure et gradine. L'arrière est dégrossi au pic.

Apí $\sigma \tau \alpha \rho \chi \circ \varsigma$

L'inscription est gravée en haut du fût, à $3,9 \mathrm{~cm}$ $\mathrm{du}$ couronnement. Elle n'est pas tout à fait centrée (marge à gauche : 1 ; à droite : 0,6). Lettres assez fines, régulières, soignées. Hauteur des lettres : 2 (alpha, tau), 2,4 (rhô, iota, sigma, chi), 1,9 (omicron).

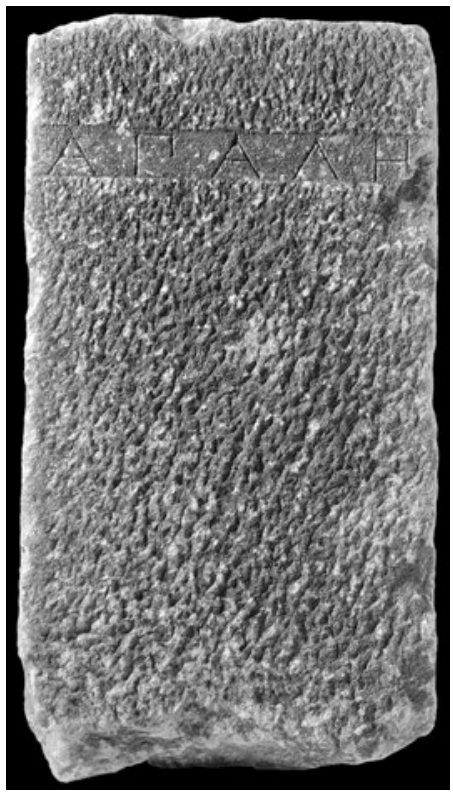

Fig. I 8 - Stèle 2

(cl. ESAG, A. Skiadaressis).

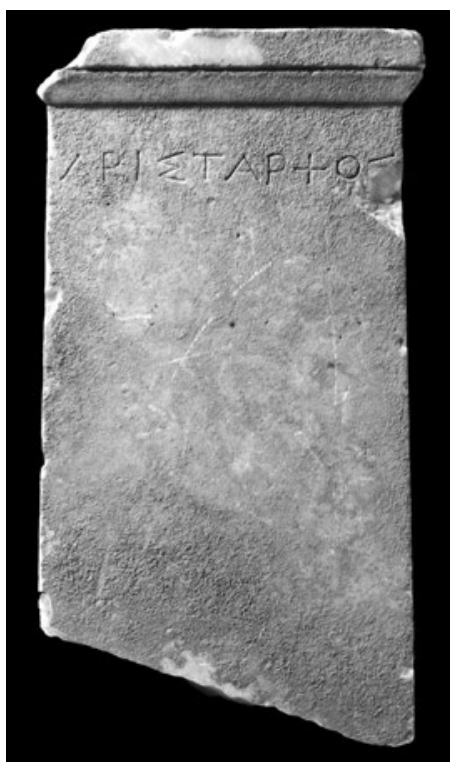

Fig. 19 - Stèle 3

(cl. ESAG, A. Skiadaressis). 
Espacement : 0,9 (alpha-rhô), 1,3 (rhô-iota; cet espacement assez grand s'explique par le iota), 1,4 (iota-sigma; même remarque), 0,8 (sigma-tau), 0,3 (tau-alpha), 0,6 (alpha-rhô, rhô-chi), 0,7 (chiomicron, omicron-sigma).

Forme des lettres : alpha à barre droite, omicron plus petit que la plupart des lettres mais presque de la même taille que l'alpha et le tau, sigma aux branches écartées, chi en forme de croix.

Fin du ves.

\section{4) Dèmètria (fig. 20)}

ME 20059.

\section{Marbre gris clair.}

63,5 × 29,4-31 × 7-9. Stèle pyramidante; elle s'épaissit légèrement vers le bas.

Stèle à sommet triangulaire, complète. Sommet du triangle abîmé; quelques éclats sur les bords de la stèle. Concrétions abondantes dans la moitié inférieure de la stèle. Face antérieure parée entièrement au ciseau grain d'orge et à la ripe plate; ciselure périmétrique $(1 \mathrm{~cm})$, moins visible à gauche. Les côtés sont travaillés au ciseau grain d'orge; une ciselure se trouve uniquement sur le côté gauche semble-t-il. Larrière est très grossièrement piqué, presque brut.

Type Dunant II A.

$\Delta \eta \mu \eta \tau \operatorname{cí}^{\alpha}$

L'inscription est gravée à $14 \mathrm{~cm}$ du sommet de la stèle. Elle n'est pas parfaitement centrée (marge à gauche : 0,6; à droite : 0,3), et l'alpha final déborde sur la ciselure périmétrique, ce qui témoigne d'une mauvaise gestion de l'espace de la part du lapicide. Grandes lettres assez épaisses, belles et régulières. Hauteur des lettres parfaitement régulière: 3,2. Espacement régulier : 0,9-1,2.

Forme des lettres : épaississements, alpha à barre droite, $m u$ aux branches écartées.

Fin du $\mathrm{IV}^{\mathrm{e}} \mathrm{s}$.

\section{5) Dokimos (fig. 21)}

\section{ME 20060.}

Marbre gris clair.

$63,8 \times 27,5-28 \times 8,4-6,6$. Stèle légèrement pyramidante; épaisseur très irrégulière.

Stèle à sommet rectiligne, complète sauf au niveau de l'angle supérieur gauche, amputé (la forme de

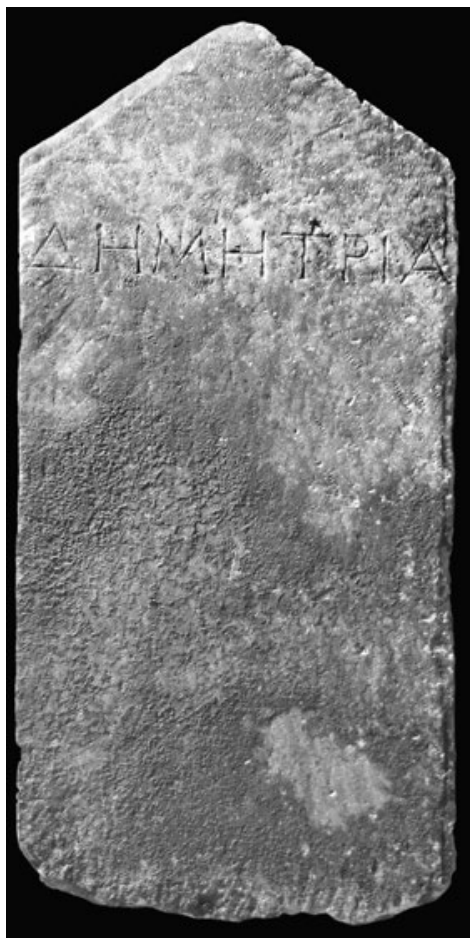

Fig. 20 - Stèle 4

(cl. ESAG, A. Skiadaressis). 
l'angle inférieur gauche ne semble pas résulter d'une cassure). Quelques éclats dans les bords. Concrétions assez abondantes sur toute la face antérieure. Face antérieure dégrossie au ciseau grain d'orge, bandeau lisse $(3,5 \mathrm{~cm})$ réalisé au ciseau pour l'inscription. Ciselure périmétrique $(1 \mathrm{~cm})$. Les côtés sont aménagés au ciseau grain d'orge; le côté droit semble même ripé. Larrière est grossièrement piqué.

Type Dunant I B.

$\Delta$ о́кцо

L'inscription est gravée à $7,2 \mathrm{~cm}$ du sommet de la stèle. Elle est parfaitement centrée (marges gauche et droite : 1,4$)$. Hauteur des lettres assez régulière $: 3$, sauf omicron $(2,6)$ et iota $(2,8)$. Espacement assez régulier : 1,4 (delta-omicron), 1,7 (omicron-kappa, kappa-iota, iota-mu), 1,2 (mu-omicron), 1,5 (omicron-sigma).

Forme des lettres: épaississements, $m u$ aux hastes quasiment droites (celle de droite est légèrement écartée), omicron plus petit, sigma aux hastes droites avec cependant une légère courbure des hastes supérieure et inférieure.

Début du $\mathrm{III}^{\mathrm{e}} \mathrm{s}$.

\section{6) Empédonikè (fig. 22)}

ME 20042.

Marbre gris clair.

$29,3 \times 22,6-23,5 \times 8,3-5,5$. Légèrement pyramidante; elle s'amincit vers le bas.

Stèle à sommet rectiligne brisée en bas sur toute la largeur et en bas à droite. Quelques éclats sur la face antérieure sur les bords. Face antérieure dégrossie à la broche; bandeau lisse $(3,5 \mathrm{~cm})$ réalisé au ciseau pour l'inscription, avec quelques traces de ripe à dents à la lisière inférieure; ciselure périmétrique (env. $1 \mathrm{~cm}$ ). Les côtés sont travaillés à la gradine. L'arrière est très grossièrement piqué.

Type Dunant I B.

'Е $\mu \pi \varepsilon \delta о v^{\prime \kappa} \kappa$

Linscription est gravée à $5,5 \mathrm{~cm}$ du sommet de la

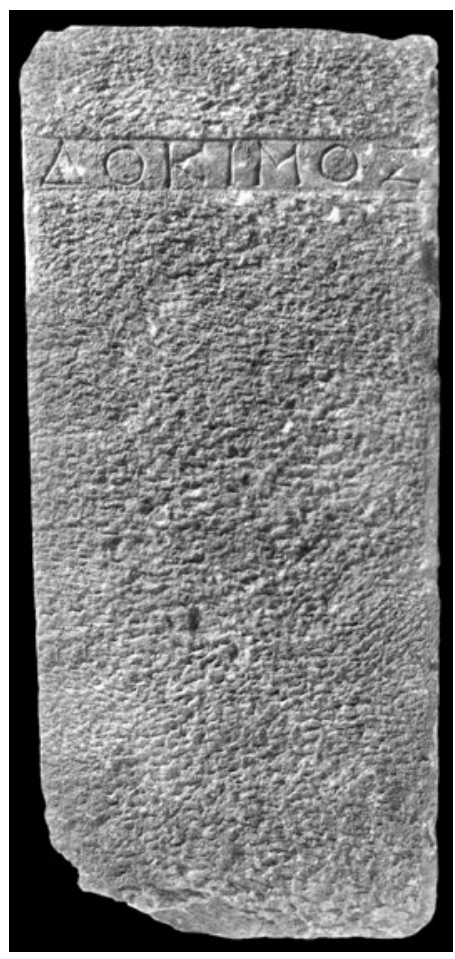

Fig. 2 I - Stèle 5 (cl. ESAG, A. Skiadaressis).

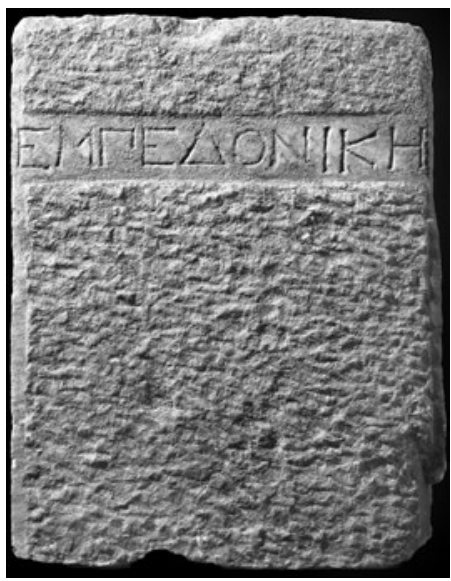

Fig. 22 - Stèle 6 (cl. ESAG, A. Skiadaressis). 
stèle. Elle est parfaitement centrée (marges gauche et droite : 0,4), mais elle déborde des deux côtés sur la ciselure périmétrique. Lettres assez épaisses, régulières, soignées. Hauteur des lettres : 2,3 (epsilon), 2,4 (mu, delta, kappa, êta), 2,1 (pi), 2,2 (second epsilon, nu), 2 (omicron), 2,6 (iota). Espacement : 0,3 (sauf pi-epsilon: 0,4, nu-iota : 0,8, iota-kappa: 0,7, kappa-êta: 0,6).

Forme des lettres: épaississements, epsilon à haste horizontale médiane plus courte, $m u$ aux branches droites, $n u$ légèrement déséquilibré, omicron à peine plus petit, $p i$ à haste verticale droite s'interrompant à mi-hauteur.

Fin du IV ${ }^{\mathrm{e}}$-début du $\mathrm{III}^{\mathrm{e}} s$.

\section{7) Eurôpa (fig. 23)}

ME 20052.

Marbre gris-bleu.

56 (avec le couronnement) $\times$ 43,6-44,4 × 6,5-8,2. Stèle légèrement pyramidante, plus épaisse à droite qu'à gauche, en raison de l'irrégularité de la face arrière.

Le couronnement est formé d'une corniche (composée d'un bandeau saillant $[1,7 \mathrm{~cm}]$ et d'un chanfrein droit $[1,5 \mathrm{~cm}])$, surmontée d'un fronton à acrotères. Le fronton, en creux, est bordé dans ses parties obliques d'un listel saillant $(1 \mathrm{~cm})$ et d'un quart de rond droit $(1 \mathrm{~cm})$. Hauteur du fronton conservée : 18. Hauteur de l'acrotère droit : 7,7.

Stèle à fronton, amputée d'une grande partie à gauche, ce qui a fait disparaître l'acrotère gauche et le début de l'inscription. L'acrotère sommital est brisé en grande partie. Quelques éclats sur les acrotères sommital et droit, et sur la face antérieure sur les bords de la stèle. Concrétions, surtout dans les trois quarts supérieurs de la stèle. Face antérieure parée à la ripe à dents. Sur les côtés, ciselure et gradine. L'arrière est très grossièrement piqué.

Type Dunant II C”.

[E]

L'inscription est gravée à $7,5 \mathrm{~cm}$ du couronnement. Elle semble centrée (distance bord gauche-upsilon: env. 8,5; marge à droite: 3,5). Grandes lettres assez fines, régulières, soignées. Hauteur des lettres remarquablement régulière: $3,7 \quad(3,1$ oméga $)$. Espacement régulier : 3,4-3,6.

Forme des lettres: épaississements, courbures de quelques lettres (hastes obliques du upsilon, haste verticale gauche du $p i$ ), alpha à barre droite, $p i$ à haste

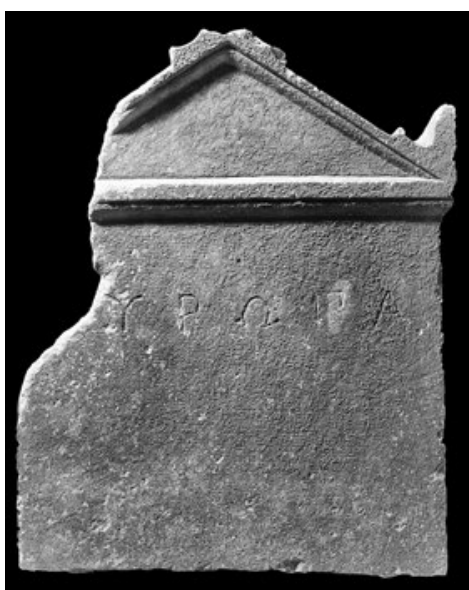

Fig. $23-$ Stèle 7

(cl. ESAG, A. Skiadaressis). verticale droite courte, oméga en arc de cercle outrepassé avec deux hastes obliques. $1^{\text {re }}$ moitié du III ${ }^{\mathrm{e}} \mathrm{s}$. 


\section{8) Kléagora (fig. 24)}

ME 20061.

Marbre gris clair.

$62 \times 33,4 \times 6,3-8,1$. La stèle n'est pas pyramidante, ce qui est plutôt rare.

Stèle à sommet triangulaire, écornée dans l'angle inférieur gauche. Grands éclats sur le côté gauche, en bas à droite et dans l'angle droit du triangle. Éclats sur la face antérieure dans les bords. Concrétions abondantes sur toute la face antérieure de la stèle. Face antérieure surfacée entièrement à la ripe à dents, sauf le quart inférieur, ciselé au ciseau grain d'orge et broché. Sur les côtés, ciselure et ciseau grain d'orge. Surface supérieure piquée finement, avec une ciselure. L'arrière est très grossièrement piqué.

Type Dunant II A.

\section{$\mathrm{K} \lambda \varepsilon \alpha \gamma o ́ \rho \alpha$}

L'inscription est gravée à $16,5 \mathrm{~cm}$ du sommet du triangle. Elle est fortement décentrée à gauche (marge à gauche: 1,3; à droite : 2,6). Lettres assez épaisses, régulières, soignées. Hauteur des lettres : 3,2 (kappa), 2,5 (lambda), 2,1 (epsilon), 2,3 (alpha, gamma, rhô), 1,9 (omicron). Espacement: 1,8 (sauf alpha-gamma, rhô-alpha: 2, gamma-omicron: 1,4, omicron-rhô : 2,5).

Forme des lettres : alpha à barre droite; epsilon à haste horizontale médiane plus courte; omicron plus petit; quelques lettres un peu courbes (kappa, lambda, gamma). $2^{\mathrm{e}}$ moitié $\mathrm{du} \mathrm{IV}^{\mathrm{e}} \mathrm{s}$.

\section{9) Mélitè (fig. 25)}

ME 20094.

Calcaire blanc.

$50 \times 27-31 \times 8,5-13$. Stèle pyramidante, plus épaisse en bas.

Stèle à sommet triangulaire, complète. La pierre est de mauvaise qualité : sa porosité a engendré des cavités sur la face antérieure, au milieu à droite et en

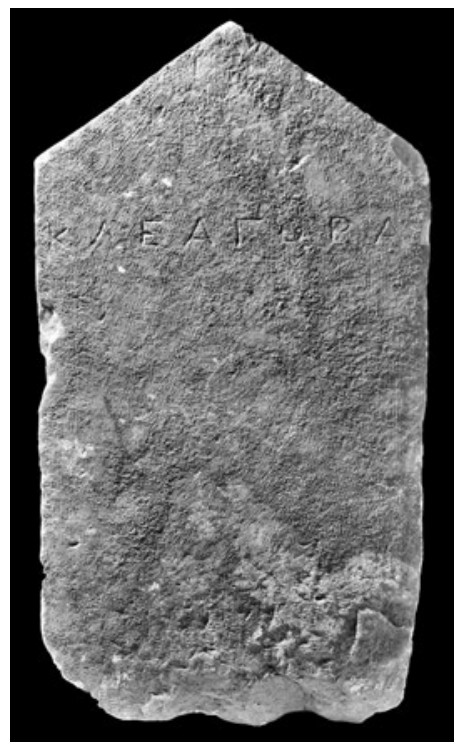

Fig. 24 - Stèle 8

(cl. ESAG, A. Skiadaressis).

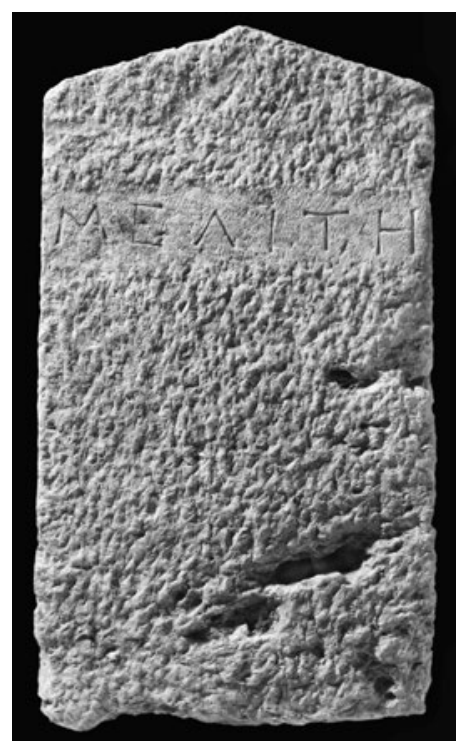

Fig. 25 - Stèle 9

(cl. ESAG, A. Skiadaressis). 
bas. Concrétions assez abondantes. Face antérieure dégrossie à la broche, bandeau lisse $(4,5 \mathrm{~cm})$ réalisé au ciseau grain d'orge pour l'inscription. Pas de ciselure périmétrique. Les côtés sont brochés. L'arrière est grossièrement piqué.

Type Dunant II B.

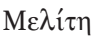

Linscription est gravée à $11,5 \mathrm{~cm}$ du sommet de la stèle. Elle est parfaitement centrée (marges gauche et droite : 1,2). Lettres fines, soignées, mais pas parfaitement alignées (surtout le $m u$ et l'epsilon). Hauteur des lettres : 2,7, sauf iota (3) et êta (3,3). Espacement : 1,7 (mu-epsilon), 2,2 (epsilon-lambda), 2,5 (lambda-iota), 2 (iota-tau, tau-êta).

Forme des lettres : épaississements, epsilon à haste médiane plus courte, $m u$ aux branches écartées. Fin du IV $s$.

\section{0) Xénarétè (fig. 26)}

\section{ME 20062.}

Marbre gris clair.

$58 \times 35,4-36,5 \times 7,3-11$. Stèle très légèrement pyramidante, plus épaisse en bas.

Stèle à sommet triangulaire, brisée dans l'angle inférieur gauche, dans l'angle inférieur droit et au sommet. Quelques éclats dans les bords. Quelques concrétions. Face antérieure dégrossie au ciseau grain d'orge, bandeau lisse $(4,7 \mathrm{~cm})$ réalisé au ciseau pour l'inscription, ciselure périmétrique $(1 \mathrm{~cm})$. Les côtés sont travaillés au ciseau grain d'orge. L'arrière est dégrossi au pic.

Type Dunant II B.

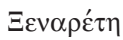

L'inscription est gravée à $16,5 \mathrm{~cm}$ du sommet (supposé) de la stèle. Elle est presque parfaitement centrée (marge à gauche: 1 ; à droite: 0,7). Grandes lettres fines, régulières, soignées. Hauteur des lettres : 4 , sauf pour le tau, le second epsilon et le rhô $(3,8)$. Espacement : 1,1 (xi-epsilon), 1,2 (epsilon-nu), 0,7 (nu-alpha, alpha-rhô, rhô-epsilon), 0,9 (epsilon-tau, tau-êta).

Forme des lettres: épaississements, alpha à barre droite, epsilon à haste horizontale centrale plus courte et à haste verticale légèrement courbe, $n u$ équilibré, $x i$ à haste verticale et à haste horizontale médiane plus courte.

Fin du IV ${ }^{\mathrm{e}}$-début du $\mathrm{III}^{\mathrm{e}} \mathrm{s}$.

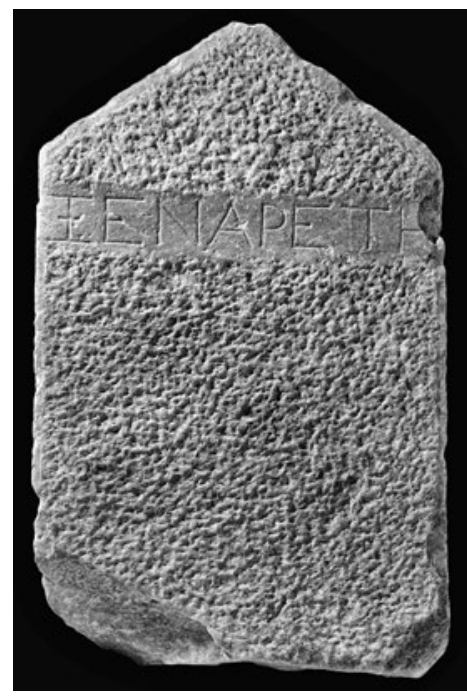

Fig. 26 - Stèle 10

(cl. ESAG, A. Skiadaressis). 


\section{1) Xénophilos (fig. 27)}

ME 20050

Micaschiste gris.

56-56,3 × 26,3-26 × 6,2-6,5. Stèle non pyramidante; épaisseur régulière en raison du type de pierre utilisé.

Stèle à sommet rectiligne, complète à part quelques éclats sur la face antérieure sur les bords. Dépressions dans la partie supérieure gauche, et dépression circulaire en bas à droite. Face antérieure parée entièrement au ciseau grain d'orge. Ciselure périmétrique visible par endroits sur le bord supérieur et sur le bord gauche. Le côté droit est aménagé (ciselure et piquage assez grossier) sur $38 \mathrm{~cm}$ seulement; probablement qu'audelà, la pierre était fichée en terre ou dans un socle. Même aménagement pour la surface supérieure, mais sans ciselure. Le côté gauche n'est pas travaillé. L'arrière a été aménagé exactement comme la face antérieure.

Type Dunant I B.

\section{$\Xi \varepsilon v o ́ \varphi \imath \lambda \circ \varsigma$}

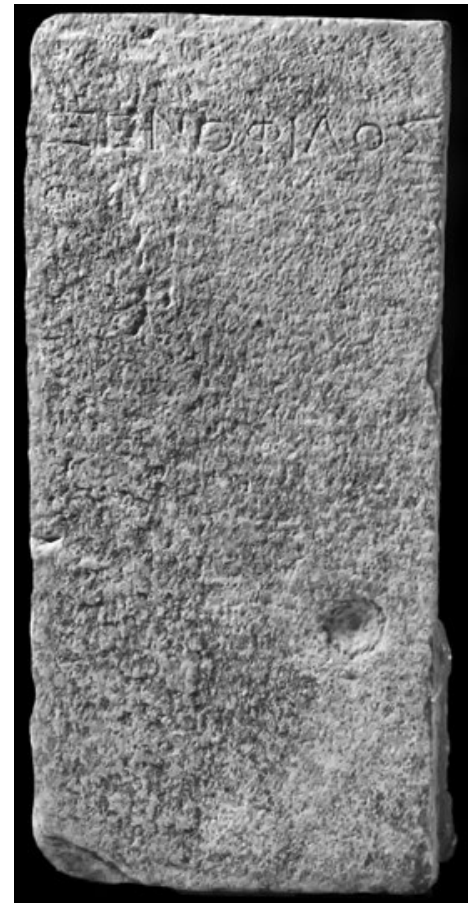

Fig. 27 - Stèle 11

(cl. ESAG, A. Skiadaressis).

L'inscription est gravée à $6,5 \mathrm{~cm}$ du sommet de la stèle. Elle est décentrée à droite (marge à gauche : 2 ;

à droite : nulle). Lettres fines, gravées remarquablement soigneusement et régulièrement compte tenu de la surface, qui n'est pas lisse. Hauteur des lettres : 2,1 (xi, epsilon, premier omicron), 2,5 ( $n u$, phi, lambda, sigma, iota), 1,7 (second omicron). Espacement régulier : 1 (sauf epsilon-nu: 1,5). Forme des lettres : epsilon à haste horizontale centrale plus courte et à haste verticale dépassant légèrement en haut, $n u$ équilibré, $x i$ à haste centrale plus courte et sans haste verticale, second omicron plus petit, sigma aux branches quasiment horizontales (la haste supérieure est légèrement oblique), phi ovale.

Fin du $\mathrm{IV}^{\mathrm{e}} \mathrm{s}$.

\section{2) Peithagorè (fig. 28)}

ME 20049.

Marbre gris clair.

49,5 × 31,4-32,7 × 5,1-9,5. Stèle pyramidante, plus épaisse en bas.

Stèle à sommet rectiligne, amputée aux angles supérieurs gauche et droit, et brisée dans sa partie inférieure droite. Quelques éclats sur la face antérieure dans les bords, dont un grand en haut à 
gauche, qui a ôté la haste verticale du pi. Concrétions abondantes; dépressions au milieu à gauche. Face antérieure dégrossie à la broche; bandeau lisse $(3,8$ $\mathrm{cm})$ aménagé au ciseau pour l'inscription; ciselure périmétrique $(1 \mathrm{~cm})$. Les côtés sont travaillés au ciseau grain d'orge. L'arrière est très grossièrement piqué.

Type Dunant I B.

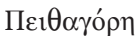

L'inscription est gravée à $8,8 \mathrm{~cm}$ du sommet de la stèle. Elle n'est pas parfaitement centrée (marge à gauche : 1,5 ; à droite : 2). Lettres fines, régulières, soignées (à part le omicron et le thêta, un peu maladroits). Hauteur des lettres : 2,1 (omicron), 2,3 (thêta), 2,6 (epsilon, iota), 3 (alpha, gamma, êta). Espacement remarquablement régulier : 1,5.

Forme des lettres: petits traits rectilignes aux extrémités; lettres rondes plus petites; alpha à barre droite; epsilon à haste horizontale médiane courte; thêta avec un point à l'intérieur; $p i$ à haste verticale droite courte.

Fin du IV -début du $\mathrm{III}^{\mathrm{e}} s$.

\section{3) Simias (fig. 29)}

ME 20056.

Marbre gris-bleu.

$51,8 \times 26,2-31,3 \times 9,2-9,6$. Stèle fortement pyramidante, plus épaisse en bas.

Stèle à sommet rectiligne, complète. Quelques éclats sur la face antérieure dans les bords et dans l'angle inférieur gauche. Concrétions abondantes quasiment sur toute la face antérieure. Face antérieure aménagée à la broche, bandeau lisse $(4 \mathrm{~cm})$ réalisé au ciseau pour l'inscription, ciselure périmétrique (env. $1 \mathrm{~cm}$ ). La partie qui était fichée en terre ou dans un socle (hauteur env. 5,5-6,5) est plus épaisse de quelques millimètres; elle est dégrossie à la broche comme le reste de la face antérieure, et la ciselure périmétrique

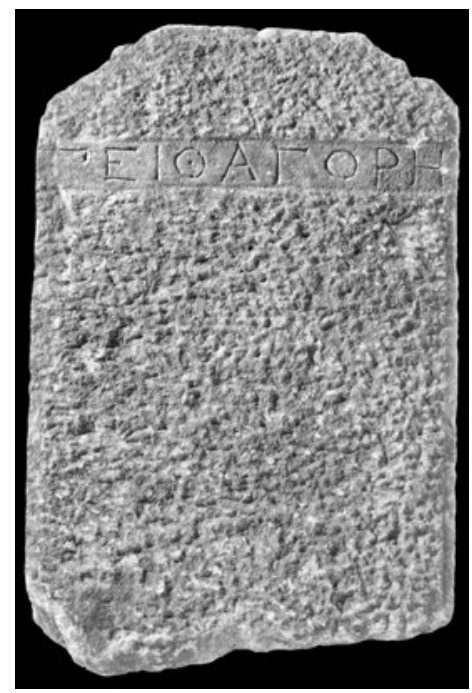

Fig. 28 - Stèle 12

(cl. ESAG, A. Skiadaressis).

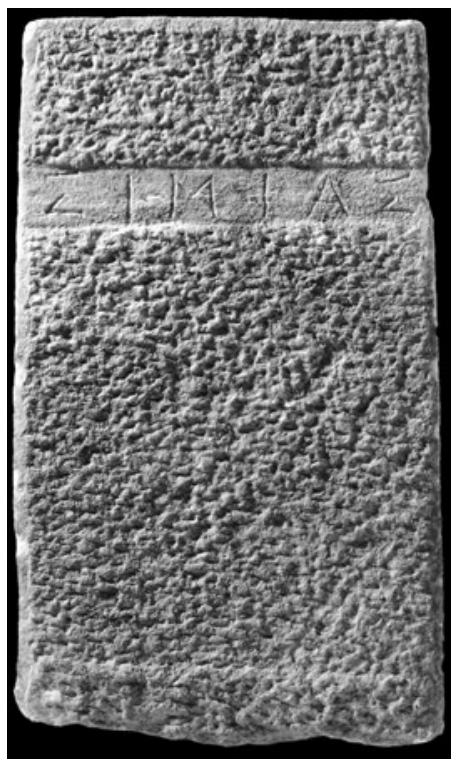

Fig. 29 - Stèle 13

(cl. ESAG, A. Skiadaressis). 
se prolonge même à son niveau. Les côtés sont travaillés au ciseau grain d'orge. L'arrière est très grossièrement piqué. Type Dunant I B.

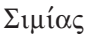

L'inscription est gravée à $9,5 \mathrm{~cm}$ du sommet de la stèle. Elle est assez bien centrée (marge à gauche : 1,9; à droite : 1,5). Lettres fines, régulières, soignées. Hauteur des lettres : 3,1 (2,9 pour le dernier sigma). Espacement : 2,9 sigma-iota, 2,5 iota-mu, 3,2 mu-iota, 2,7 iota-alpha, 1,7 alpha-sigma.

Forme des lettres : épaississements aux extrémités des sigma, alpha à barre droite, mu aux hastes quasiment droites, premier sigma aux hastes droites, second sigma aux hastes légèrement écartées et aux hastes supérieure et inférieure courbes.

Fin du $\mathrm{IV}^{\mathrm{e}}$-début du $\mathrm{III}^{\mathrm{e}} \mathrm{s}$.

\section{4) Simos (fig. 30)}

ME 20047.

Calcaire gris clair.

48,7 × 36,7 × 6-20. L'épaisseur est très irrégulière à cause de l'arrière, laissé brut.

Cippe à sommet rectiligne, complet, à part l'angle supérieur gauche (amputé) et l'angle supérieur droit (éclats sur la face antérieure). Concrétions assez abondantes. La pierre est de mauvaise qualité, elle comporte des dépressions en de multiples endroits, surtout dans les deux tiers supérieurs. Face antérieure dégrossie à la broche, bandeau lisse pour l'inscription $(6-6,5 \mathrm{~cm})$ réalisé au ciseau grain d'orge. Une ciselure périmétrique (env. $4 \mathrm{~cm}$ ) a été aménagée au ciseau grain d'orge sur le bord supérieur, en haut du bord gauche et en haut du bord droit (elle s'arrête après une dizaine de centimètres). La surface supérieure est travaillée au ciseau grain d'orge. Les côtés sont aménagés plus grossièrement (ciselure au ciseau grain d'orge mais pas sur toute la longueur, et brochage très grossier). L'arrière est brut.

Type Dunant I B.

Eînos

L'inscription est gravée à $5 \mathrm{~cm}$ du sommet du cippe. Elle est décentrée à droite (marge à gauche: 4,7; à droite: 3,3). Lettres fines, régulières, soignées. Hauteur des lettres parfaitement régulière: 3,5. Espacement assez irrégulier : 5 (sigma-iota), 3,8 (iotamu, mu-omicron), 2,5 (omicron-sigma).

Forme des lettres : mu et sigma aux branches écartées, omicron pas plus petit.

$1^{\text {re }}$ moitié du IV ${ }^{\mathrm{e}} s$.

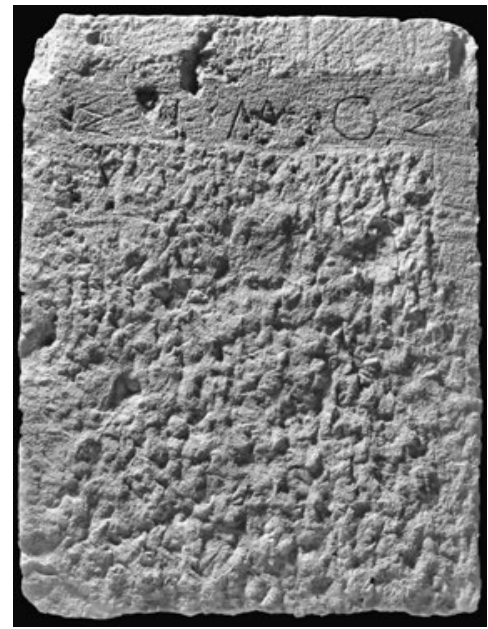

Fig. 30 - Cippe 14

(cl. ESAG, A. Skiadaressis). 
15) Phanos (fig. 31)

ME 20063.

Calcaire gris clair.

$49 \times 29-32 \times 9-15$. Cippe pyramidant; l'épaisseur est très irrégulière.

Cippe à sommet rectiligne, complet sauf l'angle supérieur droit, brisé. Quelques éclats sur les bords. La pierre, de mauvaise qualité, comporte des dépressions et des fissures en divers endroits, notamment en haut à gauche, là où se trouve une partie de l'inscription. Face antérieure aménagée à la gradine dans la partie supérieure pour recevoir l'inscription; partie inférieure dégrossie à la broche (pas sur toute la largeur du cippe cependant), puis laissée brute. Les côtés sont travaillés à la gradine, puis laissés bruts. L'arrière est brut.

Type Dunant I A.

Фâvos

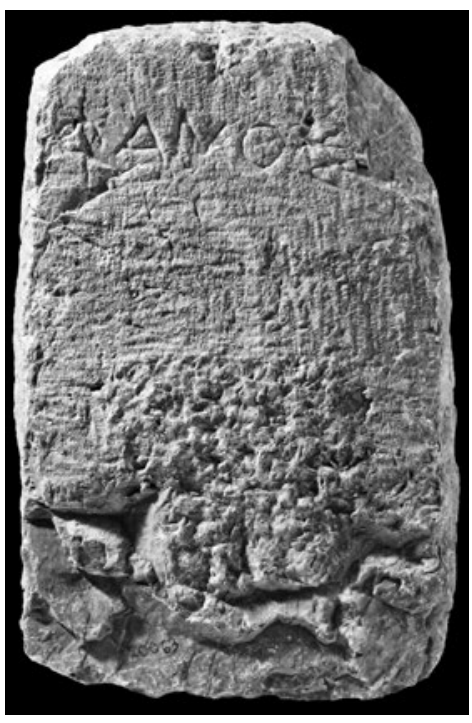

Fig. 3 I - Cippe 15

(cl. ESAG, A. Skiadaressis).

Linscription est gravée à 5-6 cm du sommet du cippe.

Elle n'est pas gravée droit : elle s'infléchit vers le bas. Elle est très fortement décentrée à gauche (marge à gauche : 1,5; à droite : 5,5). Les lettres sont épaisses, assez irrégulières et maladroites.

Hauteur des lettres très irrégulière : 5,5 (phi), 3,5 (alpha), 3,1 (nu), 2,9 (omicron), 4,2 (sigma). Espacement: 1 (phi-alpha, alpha-nu), 1,1 (nu-omicron), 2 (omicron-sigma). L'espacement est régulier, sauf pour les deux dernières lettres, plus espacées; le lapicide a peut-être réalisé qu'il allait créer une marge beaucoup trop importante à droite et tenté ainsi de réduire le déséquilibre.

Forme des lettres : alpha à barre droite, $n u$ déséquilibré, omicron un peu plus petit (mais il est presque de la même taille que le $n u$ ), sigma aux branches écartées, phi un peu ovale.

$1^{\text {re }}$ moitié du IV $s$.

\section{6) Philokratès (fig. 32)}

ME 20086.

Marbre gris-bleu.

$57 \times 34,9-35,1 \times 8,1-8,8$. Stèle très légèrement pyramidante, plus épaisse en bas.

Stèle à sommet triangulaire, complète. Deux gros éclats sur le côté droit (dont un a fait sauter la dernière lettre de l'inscription), un éclat moyen sur le côté droit du triangle, un grand éclat oblique sur le côté gauche qui a abîmé la première lettre de l'inscription, et quelques petits éclats sur le pourtour. Concrétions abondantes; dépressions au milieu à droite. Face antérieure dégrossie au ciseau grain d'orge puis à la broche fine en bas; bandeau lisse aménagé au ciseau pour l'inscription 
$(3,3 \mathrm{~cm})$, avec quelques traces de ciseau grain d'orge à la lisière; ciselure périmétrique $(1-1,2 \mathrm{~cm})$. Sur les côtés, fine ciselure et ciseau grain d'orge. L'arrière est dégrossi au pic.

Type Dunant II B.

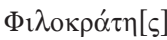

L'inscription est gravée à $16 \mathrm{~cm}$ du sommet du triangle. Elle paraît bien centrée (marge à gauche : 1,5; distance êta-bord droit: 5,3). Lettres fines, régulières, assez profondes.

Hauteur des lettres assez régulière: 2,4-2,6 (omicron : 2,1). Espacement assez régulier : 1,6 (phiiota; kappa-rhô), 1,2 (iota-lambda), 1,3 (lambdaomicron), 1,5 (omicron-kappa), 1 (rhô-alpha, alphatau), 1,7 (tau-êta).

Forme des lettres : légers apices, alpha à barre droite, kappa aux hastes obliques courtes, omicron un peu plus petit, $p h i$ ovale.

Fin du $\mathrm{IV}^{\mathrm{e}}$-début du $\mathrm{III}^{\mathrm{e}} \mathrm{s}$.

\section{7) Charinos (fig. 33)}

ME 20093.

Calcaire gris clair.

$53 \times 26,5 \times 14-21$. Épaisseur très irrégulière en raison de l'arrière, laissé brut.

Cippe à sommet triangulaire complet, mais le bord gauche du triangle est abîmé; il semble dès l'origine ne pas avoir été aménagé aussi soigneusement que le bord droit. Concrétions assez abondantes; dépressions çà et là, notamment sur le iota. Face antérieure dégrossie à la broche; bandeau lisse $(3,5 \mathrm{~cm})$ réalisé au ciseau pour l'inscription, avec quelques traces de ciseau grain d'orge. Pas de ciselure périmétrique. La partie inférieure droite semble avoir été ciselée au ciseau grain d'orge, il s'agit peutêtre d'une tentative avortée d'un aménagement plus fin de la face antérieure. Le côté gauche et la surface supérieure gauche sont très grossièrement piqués. Le

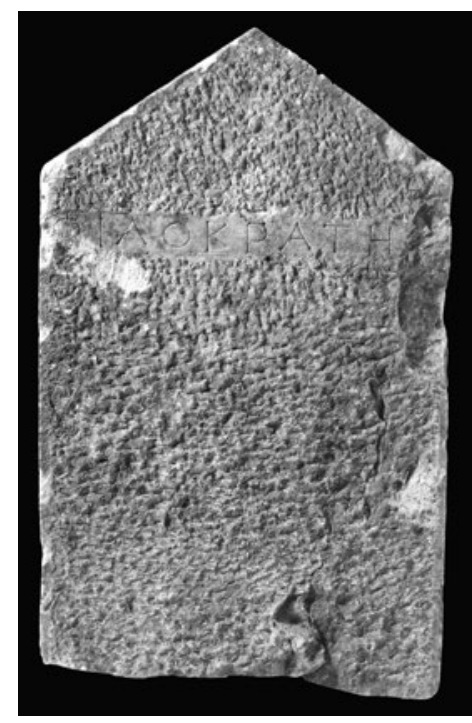

Fig. 32 - Stèle 16

(cl. ESAG, A. Skiadaressis).

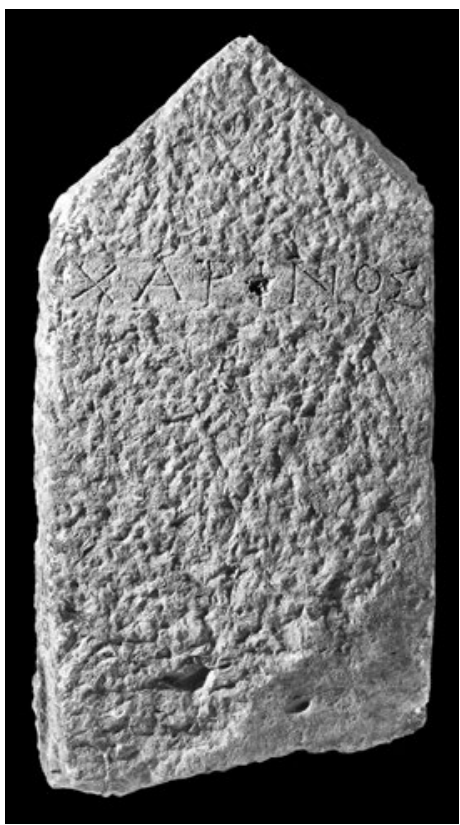

Fig. 33 - Cippe 17

(cl. ESAG, A. Skiadaressis). 
côté droit et la surface supérieure droite sont aménagés plus finement, presque lissés sur une partie de l'épaisseur. L'arrière est brut.

Type Dunant II B.

Xapîvos

Linscription est gravée à $16,5 \mathrm{~cm}$ du sommet du triangle. Elle est légèrement décentrée à droite (marge à gauche : 1,8; à droite : 0,8 ). Grandes lettres assez fines, régulières, soignées. Hauteur des lettres assez variable : 2,7 (chi, alpha), 3,3 (rhô, iota), 3 (nu), 2,1 (omicron), 3,5 (sigma). Espacement : 1,5 partout, sauf rhô-iota et iota-nu (2; cet espacement assez grand s'explique par le iota) et omicron-sigma $(0,6)$. Les deux dernières lettres sont serrées davantage et la marge à droite est plus réduite, ce qui témoigne d'une mauvaise gestion de l'espace de la part du lapicide.

Forme des lettres : épaississements, alpha à barre droite, $n u$ déséquilibré, omicron plus petit, sigma aux branches écartées.

Fin du $\operatorname{IV}^{\mathrm{e}} \mathrm{s}$

18) (Mé)lanthios (fig. 34)

ME 20055.

Marbre gris clair.

$59,5 \times 30,5-32,5 \times 5,2-8,2$. Stèle pyramidante, plus épaisse en bas.

Stèle à sommet rectiligne, complète. Gros éclat dans l'angle supérieur gauche, qui a fait sauter les premières lettres de l'inscription. Éclats dans l'angle supérieur droit. Éclat en bordure à gauche un peu en dessous du milieu de la stèle. Quelques éclats plus petits dans les bords sur la face antérieure. Concrétions abondantes. Face antérieure aménagée au ciseau grain d'orge et à la ripe à dents. Les côtés sont travaillés au ciseau grain d'orge; on observe une petite ciselure par endroits sur le côté droit. L'arrière est très grossièrement piqué.

Type Dunant I A.

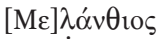

L'inscription est gravée à $5,4 \mathrm{~cm}$ du sommet de la stèle. Lettres assez épaisses, un peu maladroites (surtout les lettres rondes). De la première lettre visible, il ne reste qu'une haste oblique, qui

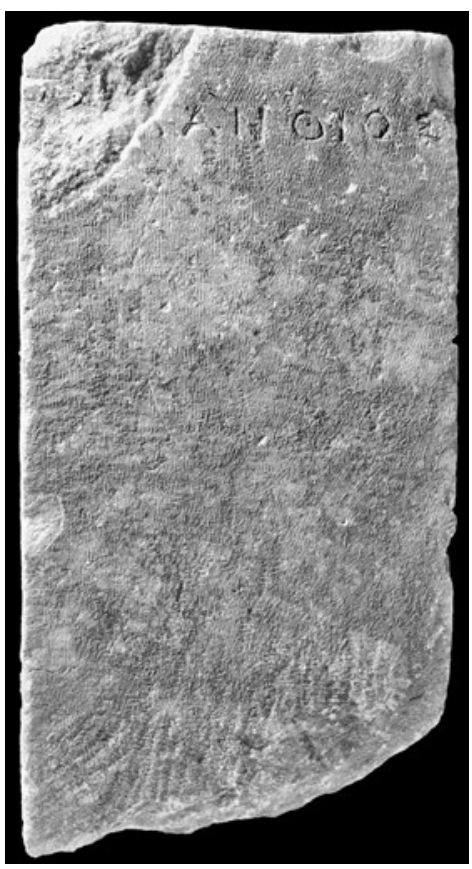

Fig. 34 - Stèle 18 (cl. ESAG, A. Skiadaressis). 
pourrait appartenir à un lambda ou à un $m u$; vu l'inclinaison de la haste et l'écartement qu'elle suppose, il s'agit plus probablement d'un lambda. Ce n'est pas la première lettre du nom : si l'on suppose la même marge à gauche qu'à droite $(1,3 \mathrm{~cm})$, on peut restituer deux lettres encore.

Hauteur des lettres assez régulière : 2,1 (alpha), 2,2 (iota, sigma), 2,3 (nu), 2 (thêta), 1,9 (omicron). Espacement assez irrégulier : 1,3 (l-alpha, alpha-nu, thêta-iota), 2,3 (nu-thêta), 1,5 (iota-omicron), 2 (omicron-sigma).

Forme des lettres : alpha à barre droite, thêta avec un point à l'intérieur, nu équilibré, sigma aux branches écartées, lettres rondes à peine plus petites.

Milieu du IV ${ }^{\mathrm{e}}$.

19) ...ôn (fig. 35)

ME 20045.

Calcaire gris clair.

$52,8 \times 26,2-28,2 \times 6,9-7,6$. Stèle pyramidante, plus épaisse en bas.

Stèle brisée en haut sur presque toute la largeur, mais le sommet semble avoir été triangulaire : on distingue l'angle supérieur droit et un segment de la pente droite du triangle. Gros éclat (ancien) en bas à gauche, petits éclats sur le bord droit. Dépressions dans le tiers supérieur gauche. La face antérieure est aménagée à la gradine et à la ripe à dents, sauf la partie inférieure qui était fichée en terre (hauteur: 13,5-14,5), seulement dégrossie à la broche. Les côtés sont travaillés à la gradine. L'arrière est grossièrement piqué.

Type Dunant II A (si le sommet est bien triangulaire).

$[--] \omega v$

Lettres fines, peu profondes. Marge à droite: 0,4. Largeur supposée de la stèle au niveau de l'inscription: 26. Largeur de l'oméga: 2,8. Largeur du $n u: 2,2$. Si l'on suppose que l'inscription occupait toute la largeur disponible, il reste encore de la place pour 4-5 lettres. Hauteur du $n u: 2,8$. Espacement oméga-nu: 2.

Forme des lettres: nu équilibré, oméga en arche de pont.

Fin du $\mathrm{IV}^{\mathrm{e}}$-début du $\mathrm{III}^{\mathrm{e}}$ s.

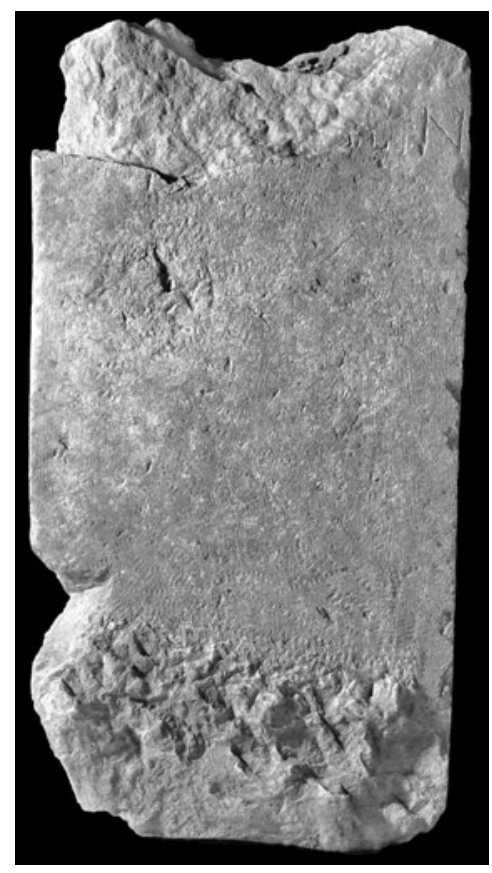

Fig. 35 - Stèle 19

(cl. ESAG, A. Skiadaressis). 


\section{0) Archémonè fille d'Eubios (fig. 36)}

ME 20112.

Calcaire marmorisé gris foncé.

47,3 × 31,4-31,9 × 5,2-5,5. Stèle pyramidante; l'épaisseur est remarquablement régulière.

Stèle à sommet triangulaire, brisée dans la partie inférieure gauche et à la base. Grand éclat en bas à droite, quelques éclats sur la face antérieure dans les bords. Dépressions au centre et en bas à droite. Face antérieure surfacée à la ripe à dents. Sur les côtés, ciselure et ciseau grain d'orge. L'arrière est très grossièrement piqué.

Type Dunant II A.

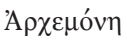

Eủßíov

L'inscription est gravée à $14,3 \mathrm{~cm}$ du sommet du triangle. Elle n'est pas parfaitement centrée (première ligne: marge à gauche 0,5 , à droite 0,8 ; seconde ligne : marge à gauche 3,4, à droite 2,6). Lettres fines, un peu maladroites.

Hauteur des lettres régulière à la première ligne : 2,3 partout, sauf epsilon $(2,2)$ et omicron $(1,8)$. Seconde ligne: 1,8 epsilon, 1,9 upsilon, 2,2 bêta, 2,3 iota, 1,6 omicron. Espacement assez irrégulier : première ligne : 2 (alpha-rhô, rhô-chi), 1,5 (chi-epsilon), 2,5 (epsilon-mu, nuêta), 2,6 (mu-omicron), 1,9 (omicron-nu). Seconde ligne : 4,5 (epsilon-upsilon), 2,9 (upsilon-bêta, iota-omicron), 3,8 (bêta-iota), 2,7 (omicron-upsilon). Interligne : env. 0,4.

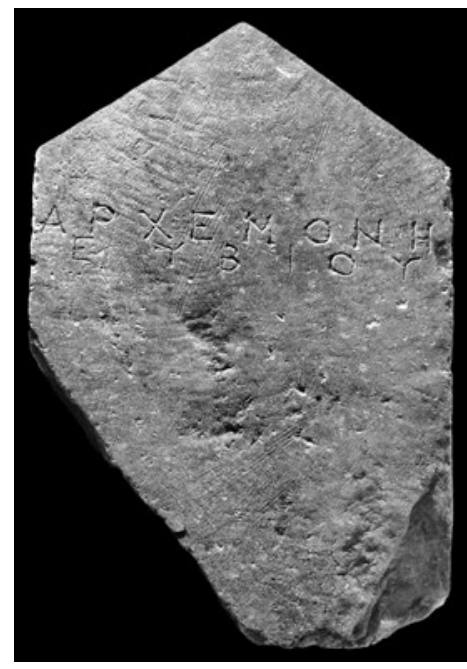

Fig. 36 - Stèle 20

(cl. ESAG, A. Skiadaressis).

Forme des lettres : quelques dépassements de la haste verticale en haut (bêta, epsilon, rhô), alpha à barre droite, epsilon à haste horizontale médiane plus courte, $m u$ aux branches droites, $n u$ légèrement déséquilibré et la haste oblique ne part pas du sommet de la haste verticale gauche, omicron un peu plus petit, le premier upsilon a des hastes obliques droites mais le second a une haste oblique droite courbe.

Début du III $s$.

\section{1) Biotè fille de Nikoklès (fig. 37)}

ME 20065.

Marbre gris clair.

75,5 (avec le couronnement) × 37-38 × 5,3-8. Stèle légèrement pyramidante.

Le couronnement est formé d'une corniche, surmontée d'un fronton pourvu d'acrotères. Corniche : listel saillant $(1 \mathrm{~cm})$, scotie renversée $(1,8 \mathrm{~cm})$, ovolo renversé $(1,7 \mathrm{~cm})$, filet 
droit $(1 \mathrm{~cm})$. Fronton en creux, bordé d'un listel saillant $(1 \mathrm{~cm})$. Hauteur des acrotères : 3 (angles) et 4 (sommet). Hauteur du fronton: 14,5. Décrochement du couronnement par rapport au fût de la stèle : 1,5 .

Stèle à fronton, complète. Quelques éclats sur la face antérieure dans les bords et sur les acrotères. Éclat allongé en haut à gauche le long du bord, qui a légèrement affecté la haste gauche du $n u$ de la seconde ligne. Très grand éclat en bas sur les deux tiers de la largeur de la base. Concrétions abondantes sur toute la face antérieure de la stèle ; dépressions au centre. Face antérieure entièrement ripée à la ripe à dents. Sur les côtés, petite ciselure (mais elle ne semble pas être présente partout) et ciseau grain d'orge. L'aménagement du côté gauche cesse vers le bas, laissant $9 \mathrm{~cm}$ bruts; le côté droit est par contre aménagé jusqu'en bas. L'arrière est très grossièrement piqué.

Type Dunant II C”.

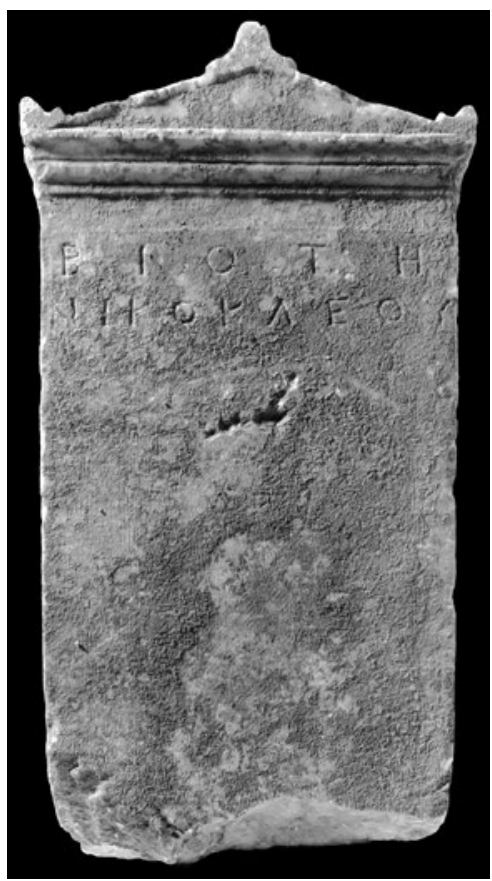

Fig. 37 - Stèle 21

(cl. ESAG, A. Skiadaressis).

Bió ๆ

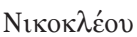

L'inscription est gravée à 4,2 cm du couronnement. Elle n'est pas parfaitement centrée (première ligne : marge à gauche 2,3 , à droite 3,7 ; seconde ligne : marge à gauche 1,3 , à droite 0,7 ). Lettres assez épaisses, régulières, soignées.

Hauteur des lettres remarquablement régulière: première ligne : 2,6 (bêta, iota, êta) et 2,5 (omicron, tau). Seconde ligne : 2,5 partout, sauf omicron et upsilon $(2,2)$.

Espacement très régulier à la première ligne, sauf les deux dernières lettres, plus espacées : 5,3 (bêta-iota, iota-omicron), 5,5 (omicron-tau), 6,2 (tau-êta). Espacement assez irrégulier à la seconde ligne : 2,4 (nu-iota), 1,9 (iota-kappa), 2 (kappa-omicron), 2,2 (omicron-kappa, kappa-lambda), 3,2 (lambda-epsilon), 2,8 (epsilon-omicron), 2,3 (omicron-upsilon). Interligne : env. 2,2 .

Forme des lettres : epsilon à haste horizontale médiane un peu plus courte, kappa aux hastes obliques un peu plus courtes, $n u$ légèrement déséquilibré, omicron de la même taille que les autres lettres à la première ligne, un peu plus petit à la seconde ligne.

Fin du IV -début du III $s$. 


\section{2) Dôrias fille de Théôn (fig. 38)}

ME 20094.

Marbre gris clair.

$64,5 \times 30,1-30,4 \times 7-7,5$. Stèle très légèrement pyramidante.

Stèle à sommet triangulaire, complète sauf l'angle gauche du triangle et l'angle inférieur droit de la stèle. Éclats au sommet du triangle, et çà et là sur les bords de la stèle. Concrétions abondantes, plus rares à droite et en bas. Face antérieure entièrement aménagée à la ripe à dents, sauf la partie fichée en terre, dégrossie à la broche (hauteur : $8,5 \mathrm{~cm}$ ) et précédée d'une petite zone au ciseau grain d'orge. Amorce d'une ciselure périmétrique d'environ $1,5 \mathrm{~cm}$ en bas à droite et à gauche, mais elle ne semble pas se prolonger plus haut (les concrétions rendent l'examen difficile). Sur les côtés, ciselure et ciseau grain d'orge. L'arrière est très grossièrement piqué.

Type Dunant II A.

$\Delta \omega \rho \grave{\alpha} \varsigma$

$\Theta \varepsilon \dot{\varepsilon} \omega \mathrm{vo \zeta}$

L. 2: on ne voit rien à l'intérieur du thêta, le point était peut-être peint.

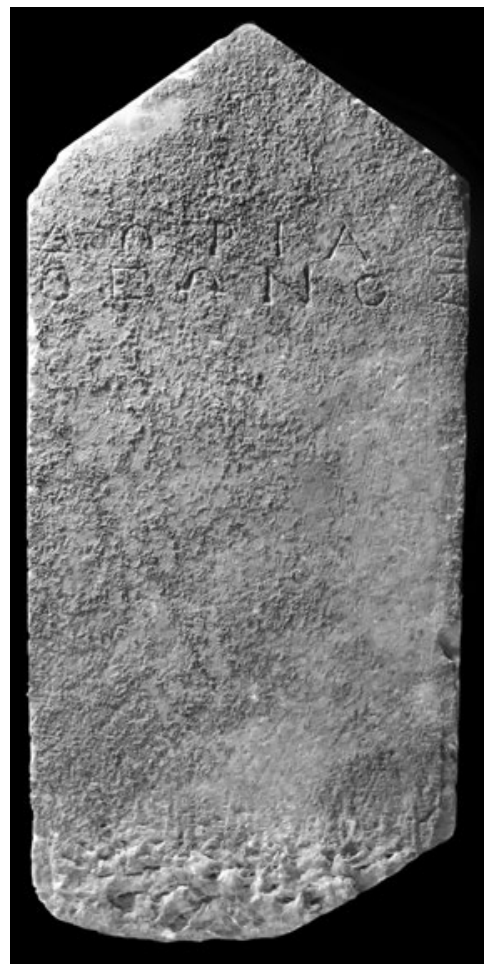

Fig. 38 - Stèle 22

(cl. ESAG, A. Skiadaressis).

Linscription est gravée à $13,5 \mathrm{~cm}$ du sommet du triangle. Elle est décentrée à droite (première ligne : marge à gauche 0,9 , à droite 0,2 ; seconde ligne : marge à gauche 0,9 , à droite 0 ). Lettres assez épaisses, régulières, soignées.

Hauteur des lettres assez régulière: 2,5 partout, sauf delta (2), oméga $(1,8)$, omicron $(2,3)$. Espacement : première ligne : 2,5 (delta-oméga), 3,3 (oméga-rhô), 3 (rhô-iota, iota-alpha), 4 (alphasigma); seconde ligne: 2,8 (thêta-epsilon, oméga-nu), 2,5 (epsilon-oméga), 3 (nu-omicron), 3,3 (omicron-sigma). Les deux dernières lettres de la première ligne ont été espacées davantage, pour que le nom occupe toute la largeur de la stèle; cela témoigne d'une mauvaise gestion de l'espace de la part du lapicide. Interligne : env. 1.

Forme des lettres : lettres rondes un peu plus petites à l'exception du thêta, alpha à barre droite et légèrement oblique, haste horizontale médiane de l'epsilon plus courte, sigma aux branches un peu écartées, nu équilibré, oméga en arche de pont ( $1^{\text {re }}$ ligne) ou ouvert et comme "suspendu", avec prolongation des lignes de l'arche en bas ( $2^{\mathrm{e}}$ ligne).

$1^{\text {re }}$ moitié du $\mathrm{III}^{\mathrm{e}} \mathrm{s}$. 


\section{3) Kall... Ma... (fig. 39)}

ME 20043.

Marbre gris-bleu.

27 (hauteur conservée) $\times 21$ (largeur conservée) $\times$ 4-4,8

Fragment supérieur gauche d'une stèle à sommet rectiligne, brisée à droite et en bas. Gros éclats en haut à droite et dans l'angle supérieur gauche. Quelques éclats sur la face antérieure dans les bords. Concrétions abondantes. La face antérieure est aménagée au ciseau grain d'orge. Les côtés gauche et droit sont grossièrement brochés. La surface supérieure est brute, ce qui est très rare. L'arrière est lisse, ce qui est également très rare.

Type Dunant I A.

$\mathrm{K} \alpha \lambda \lambda[--]$

$\mathrm{M} \alpha[--]$

Linscription est gravée à 6,5-8,5 $\mathrm{cm}$ du sommet (le sommet n'est pas plat, il est irrégulier car il n'est pas aménagé). La seconde ligne doit être plus courte que la première, d'après les marges à gauche (première ligne : 8,5; seconde ligne : 12) et l'espacement entre les lettres, plus grand qu'à la première ligne. Il doit s'agir du patronyme du défunt, mais un ethnique n'est pas exclu.

Lettres fines, soignées, assez régulières (sauf le kappa,

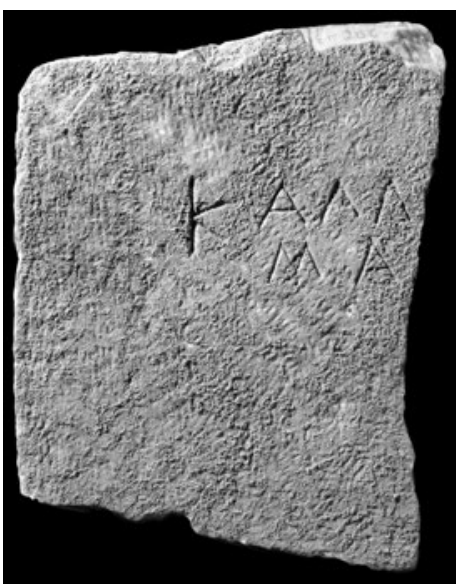

Fig. 39 - Stèle 23

(cl. ESAG, A. Skiadaressis). beaucoup trop grand et non aligné sur les autres lettres, comme s'il avait été ajouté après-coup; et le second lambda est plus long que le premier).

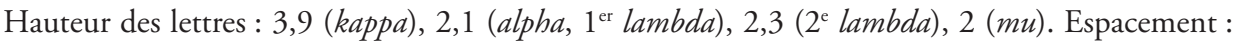
première ligne : 1,6 (kappa-alpha), 0,6 (alpha-lambda), 0,5 (lambda-lambda); seconde ligne : 1,6. Interligne : env. 0,9 .

Forme des lettres : alpha à barre droite, kappa aux hastes obliques courtes, mu aux branches écartées.

$2^{\mathrm{e}}$ moitié du IV $\mathrm{e}$.

\section{4) Léontichos fils de Symmachos (fig. 40)}

ME 20048.

Marbre gris-bleu.

$29,5 \times 32,7-34 \times 9,5-12$. Stèle pyramidante; épaisseur assez régulière.

Petite stèle à sommet rectiligne, brisée en bas à droite. Quelques éclats sur la face antérieure dans les bords, le plus grand étant dans l'angle inférieur droit. Concrétions abondantes sur toute la 
face antérieure. Face antérieure dégrossie à la broche, bandeau lisse $(6,5 \mathrm{~cm})$ réalisé au ciseau pour l'inscription, ciselure périmétrique $(1-1,5 \mathrm{~cm})$. Les côtés sont travaillés au ciseau grain d'orge. Larrière est brut.

Type Dunant I B.

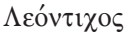

$\Sigma v \mu \mu \alpha ́ \chi 0 v$

Linscription est gravée à $8,5 \mathrm{~cm}$ du sommet. La première ligne est mieux centrée que la seconde (marge à gauche $1.1: 1$, à droite: 1,2 ; marge à gauche $1.2: 0,4$, à droite : 1,7). Lettres fines, régulières, soignées.

Hauteur des lettres : 2,5 partout, sauf omicron $(2,3)$, iota, tau, nu, alpha, second chi (2,7), upsilon (3). Espacement : 1,5-1,7, sauf chi-omicron à la seconde ligne $(1,1)$ et omicron-upsilon à la seconde ligne $(0,5)$. Les lettres de la seconde ligne se resserrent vers la

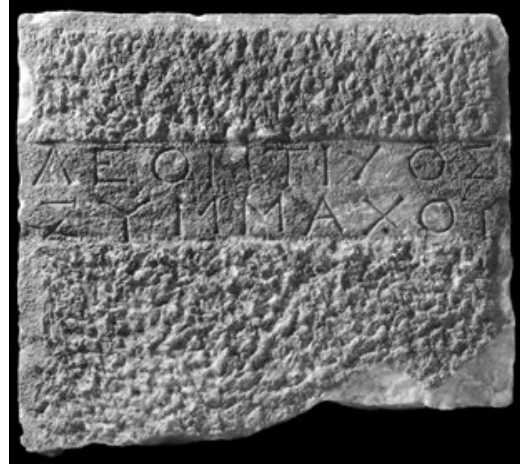

Fig. 40 - Stèle 24 (cl. ESAG, A. Skiadaressis). fin. Interligne : env. 1.

Forme des lettres : petits traits rectilignes aux extrémités, quelques légers apices ( $n u$, tau, iota), alpha à barre droite, haste horizontale médiane de l'epsilon plus courte et haste verticale dépassante en haut et en bas, nu déséquilibré, omicron à peine plus petit, sigma aux branches quasiment horizontales (la haste supérieure est légèrement oblique).

Milieu- $2^{\mathrm{e}}$ moitié du $\mathrm{III}^{\mathrm{e}} \mathrm{s}$.

\section{5) Lysimachos fils de Théodôros (fig. 41)}

ME 20097.

Marbre gris-bleu.

35 (avec le couronnement) × 29,2-30 × 4-7. Stèle pyramidante, plus épaisse en bas.

Le couronnement est composé d'une corniche, surmontée d'un fronton à acrotères. Corniche : bandeau saillant $(1,2)$, chanfrein droit $(0,6)$. Fronton en creux, bordé d'un listel saillant $(0,5)$. Hauteur de l'acrotère d'angle : 3,4; hauteur de l'acrotère sommital : 4,5 . Hauteur du fronton : 12,7. Décrochement du couronnement par rapport au fût de la stèle : 0,7 .

Fragment supérieur d'une stèle à fronton, brisée en bas. Éclats sur l'acrotère sommital et sur la pente supérieure gauche du fronton; l'acrotère droit est en partie brisé; quelques éclats dans les bords. Concrétions abondantes. Face antérieure ripée à la ripe à dents. Les côtés sont ripés comme la face antérieure, ce qui est rare. L'arrière est très grossièrement piqué. 
Type Dunant II C”.

$\Lambda v \sigma i ́ \mu \alpha \chi \sigma s$

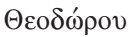

Linscription est gravée à $1,6 \mathrm{~cm}$ du couronnement. Elle est légèrement décentrée à droite (marge à gauche $1.1: 0,4$, à droite : nulle; marge à gauche 1. 2 : 0,8 , à droite : nulle). Lettres assez épaisses, régulières, soignées.

Hauteur des lettres assez régulière : première ligne : 2,3 partout, sauf chi $(2,2)$ et omicron $(1,6)$. Seconde ligne: 2,3 partout, sauf omicron (1,7), oméga $(2,1)$, rhô $(2,5)$, upsilon $(2,7)$. Espacement bien équilibré : première ligne: 1,2 (lambda-upsilon,

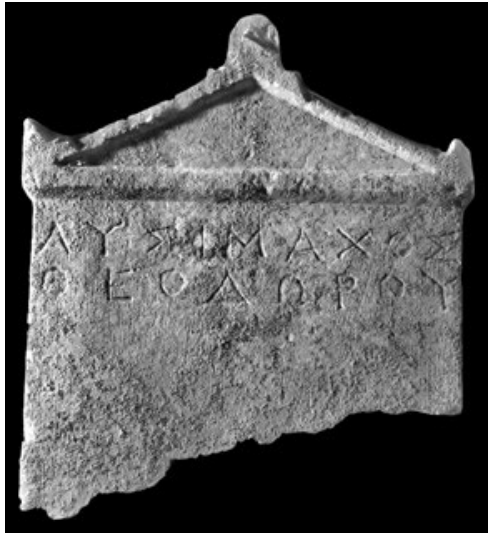

Fig. 4I - Stèle 25

(cl. ESAG, A. Skiadaressis).

alpha-chi, chi-omicron), 1,6 (upsilon-sigma), 1,5

(sigma-iota et mu-alpha), 1,3 (iota-mu), 1 (omicron-sigma). Seconde ligne : 2,8 (thêta-epsilon), 1,8 (epsilon-omicron, omicron-delta, oméga-rhô), 1,2 (delta-oméga), 1,7 (rhô-omicron), 1,3 (omicron-upsilon). Interligne : env. 1.

Forme des lettres : épaississements, lettres rondes plus petites (surtout le omicron), alpha à barre droite et légèrement oblique, haste horizontale médiane de l'epsilon un peu plus courte, thêta avec un point à l'intérieur, mu et sigma aux branches un peu écartées, oméga en arche de pont.

Début du $\mathrm{III}^{\mathrm{e}} \mathrm{s}$.

\section{6) Nikylla fille de Nikôn (fig. 42)}

ME 20053.

Marbre gris clair.

$66,5 \times 32,6-34,5 \times 9-10$ (à droite) et 9-3 (à gauche). Stèle pyramidante; épaisseur très irrégulière à gauche.

Stèle à sommet triangulaire, complète à part le sommet du triangle, amputé. Quelques éclats sur la face antérieure dans les bords, notamment dans les angles gauche et droit du triangle. Un éclat a abîmé la haste gauche de la première lettre $(n u)$, mais ne gêne pas la lecture. Concrétions abondantes, surtout dans la moitié inférieure. Face antérieure dégrossie au ciseau grain d'orge puis, plus bas, à la broche fine, bandeau lisse $(4,5 \mathrm{~cm})$ réalisé au ciseau pour l'inscription, ciselure périmétrique (env. $1,2 \mathrm{~cm}$ ). Les côtés sont travaillés au ciseau grain d'orge. Sur le côté gauche, l'aménagement, tout comme la ciselure périmétrique sur la face antérieure, cesse vers le bas, laissant $13 \mathrm{~cm}$ bruts (on n'observe pas cela à droite). L'arrière est très grossièrement piqué. 
Type Dunant II B.

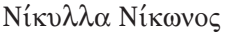

L'inscription est gravée à $14,5 \mathrm{~cm}$ du sommet du triangle. Elle est parfaitement centrée (marges gauche et droite: 0,4$)$. Lettres fines, régulières, soignées.

Hauteur des lettres régulière: 2,8 partout, sauf premier nu et oméga $(2,6)$, premier kappa, lambda, alpha, second iota (2,7), omicron $(2,3)$.

Espacement : 0,3-0,5 (nom), 1,5 (entre le nom et le patronyme), 0,5-0,6 (patronyme, sauf nuomicron $[0,4]$ et omicron-sigma $[0,3])$. Les lettres du patronyme sont plus espacées, mais les trois dernières lettres sont resserrées, ce qui témoigne d'une mauvaise gestion de l'espace de la part du lapicide. Par ailleurs, les lettres outrepassent la ciselure périmétrique de chaque côté.

Forme des lettres : épaississements, alpha à barre droite, $n u$ équilibré mais la haste oblique part sous le sommet de la haste verticale gauche, omicron un peu plus petit, sigma aux branches écartées et aux hastes inférieure et supérieure courbes, oméga très fermé

$2^{\mathrm{e}}$ moitié du IV s.

\section{7) Silènis fille d'Apollônios (fig. 43)}

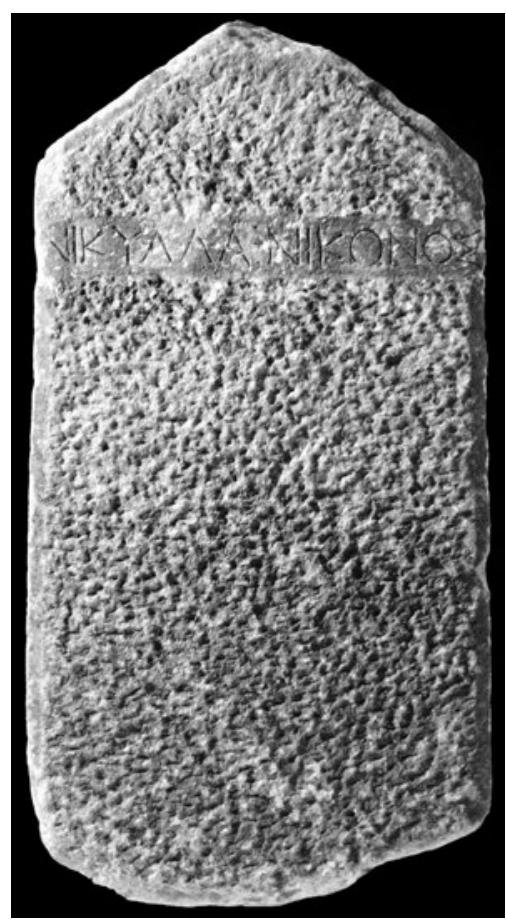

Fig. $42-$ Stèle 26

(cl. ESAG, A. Skiadaressis).

ME 20051.

Marbre gris clair.

57,5 (avec le couronnement) × 31,7-33,8 × 6,5-7,2. Stèle pyramidante, plus épaisse en bas.

Le couronnement est de même type que le numéro 7 . Corniche : bandeau saillant : 1,5; chanfrein droit : 1,8. Fronton : listel saillant : 0,5 ; quart de rond droit : 1 . Hauteur du couronnement : 11,8 . Hauteur de l'acrotère (sans le listel) : 6 .

Stèle à fronton, brisée en haut à droite. Il ne reste qu'un acrotère du fronton, celui de gauche. Quelques éclats dans les bords; gros éclats au milieu en bas, dans l'angle inférieur droit et sur l'acrotère. Concrétions abondantes. La face antérieure est entièrement surfacée à la ripe à dents. Sur les côtés, ciselure (elle n'est pas très visible à gauche à cause de concrétions) et ciseau grain d'orge. L'arrière est très grossièrement piqué. 
Type Dunant II C”.

$\Sigma 1 \lambda \eta v i[s]$

'A $\pi \mathrm{\circ} \lambda \lambda \omega v i ́ 0 v$

L'inscription est gravée à $6 \mathrm{~cm}$ du couronnement. Elle est pratiquement centrée, en tout cas pour la seconde ligne (marge à gauche $1.1: 2,1$, distance iôta-bord droit: 8; marge à gauche 1. 2 : 0,8 , à droite: env. 0,7 ). Les lettres sont fines, régulières, soignées.

Hauteur des lettres très régulière : 2,2-2,3, sauf omicron et oméga $(1,6)$. Espacement: première ligne: 1,8 (sigma-iota, lambda-êta), 2,4 (iotalambda, êta-nu), 3,4 (nu-iota); seconde ligne: 1-1,1 partout, sauf oméga-nu $(1,4)$, nu-iota $(1,9)$, iota-omicron $(1,3)$. Interligne : 1 .

À la première ligne, on ne voit rien de la lettre finale restituée; cela n'est pas impossible, si l'on suppose une marge de $2,1 \mathrm{~cm}$ (comme à gauche), un sigma de $2 \mathrm{~cm}$ de large (comme le

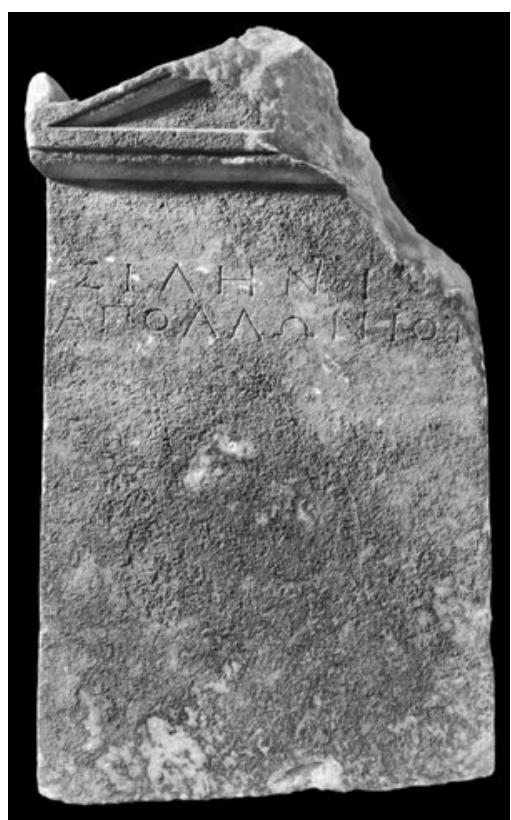

Fig. 43 - Stèle 27

(cl. ESAG, A. Skiadaressis). premier sigma), et un espacement de $4 \mathrm{~cm}$ (on remarque que le graveur a espacé de plus en plus les dernières lettres de la première ligne).

Forme des lettres : épaississements, lettres rondes plus petites, alpha à barre droite, $n u$ très légèrement déséquilibré, $p i$ à haste verticale droite courte, sigma aux branches quasiment horizontales, oméga en arche de pont.

Début du $\mathrm{III}^{\mathrm{e}} \mathrm{s}$.

\section{8) ...nippè fille d'Eumolpos (fig. 44)}

ME 20200.

Remployée dans la maçonnerie des parois latérales du niveau supérieur de la tombe T6.

Marbre gris clair.

25,6 (hauteur conservée) × 22 (largeur conservée) × 5-6.

Fragment supérieur droit d'une stèle à sommet rectiligne, brisée à gauche et en bas. Quelques éclats sur la face antérieure sur les bords. Concrétions abondantes. Face antérieure parée à la ripe à dents. Les côtés sont travaillés à la gradine. L'arrière est très grossièrement piqué. 
Type Dunant I A.

[..]vín $\pi \eta$

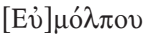

L'inscription est gravée à $4 \mathrm{~cm}$ du sommet de la stèle. Pas de marge à droite, les lettres vont jusqu'au bord de la stèle. Lettres assez épaisses, régulières, soignées.

Hauteur des lettres régulière : 1,8 partout, sauf iota, premier $p i$ à la $1^{\text {re }}$ ligne, upsilon $(1,9)$, second $p i$ à la $1^{\text {re }}$ ligne (2), omicron $(1,5)$. Espacement: première ligne: 2,1 (nu-iota, iota-pi), 2,5 (pi-pi), 2,6 (pi-êta). Seconde ligne: 1,2 (mu-omicron, omicron-lambda), 1 (lambda-pi, omicron-upsilon), 1,5 (pi-omicron). Le patronyme devait être plus long que l'idionyme car

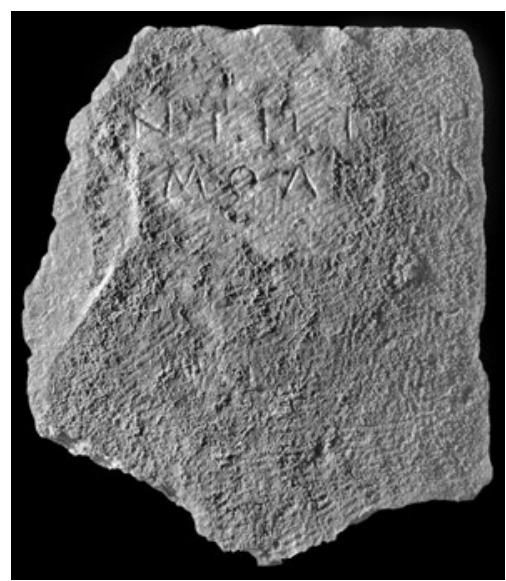

Fig. 44 - Stèle 28

(cl. ESAG, A. Skiadaressis). les espacements sont plus courts; les possibilités de restitution sont assez nombreuses pour l'idionyme, voir infra, p. 215-216. Interligne : env. 1.

Forme des lettres : $m u$ aux branches écartées, $n u$ déséquilibré, omicron plus petit, $p i$ dont la haste verticale droite descend jusqu'aux trois-quarts de la lettre et au-delà.

$2^{\mathrm{e}}$ moitié du IV $\mathrm{e}^{\mathrm{e}} \mathrm{s}$.

\section{9) Aristagoras de Cyzique (fig. 45)}

ME 20058.

Marbre gris clair.

67 (avec le couronnement) $\times 30,9-32,7 \times 7,7-8,5$. Stèle pyramidante. L'épaisseur est remarquablement régulière.

Le couronnement se compose d'une corniche, surmontée d'un fronton sculpté en relief et pourvu d'acrotères. Corniche: bandeau saillant: 2,2; chanfrein droit: 2,5. Fronton: listel saillant: 1; hauteur du fronton: 14,8. Hauteur des acrotères central et droit: 5. Décrochement du couronnement par rapport au fût de la stèle : 1 .

Stèle à fronton sculpté en relief sur le fond, brisée dans l'angle inférieur gauche et sur la base à droite. Extrémités des acrotères abîmées par des éclats. Éclats sur la face antérieure sur les bords, éclat plus gros à gauche de l'acrotère sommital. Quelques concrétions, surtout dans la partie du fût sous l'inscription. Face antérieure parée à la gradine, sauf la partie enterrée (hauteur : 4-4,5), grossièrement brochée. Le couronnement a été réalisé au ciseau. Les côtés de la stèle sont gradinés, comme la face antérieure, mais jusqu'en bas. La surface supérieure comporte une large ciselure puis est grossièrement piquée. L'arrière est très grossièrement piqué. 
Type Dunant II C'.

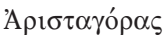

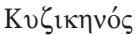

L. 1 : la barre horizontale du second alpha n'a pas été gravée.

Linscription est gravée à $0,6 \mathrm{~cm}$ du couronnement. Elle est parfaitement centrée (marges 1. 1:1,7; marges 1. $2: 3,4)$. Lettres fines, régulières, soignées.

Hauteur des lettres assez régulière: 2,2 partout, sauf omicron (1,4 première ligne, 1,5 seconde ligne), sigma de la première ligne $(2,3)$, zêta (2), kappa, iota de la seconde ligne, upsilon, nu $(2,4)$, êta, sigma de la seconde ligne $(2,5)$.

Espacement bien équilibré : première ligne: 0,9 alpha-rhô, sigma-tau, alpha-gamma, alpha-sigma; 1,6 rhô-iota; 1,5 iôta-sigma; 0,3 tau-alpha et gammaomicron; 1 omicron-rhô; 0,6 rhô-alpha. Seconde ligne : 0,5 kappa-upsilon; 0,7 upsilon-zêta; 1,4 zêta-iota et kappa-êta; 1,6 iota-kappa; 1,2 êta-nu et nu-omicron; 1,5 omicron-sigma. Interligne : env. 1.

Forme des lettres : alpha à barre droite, zêta en forme

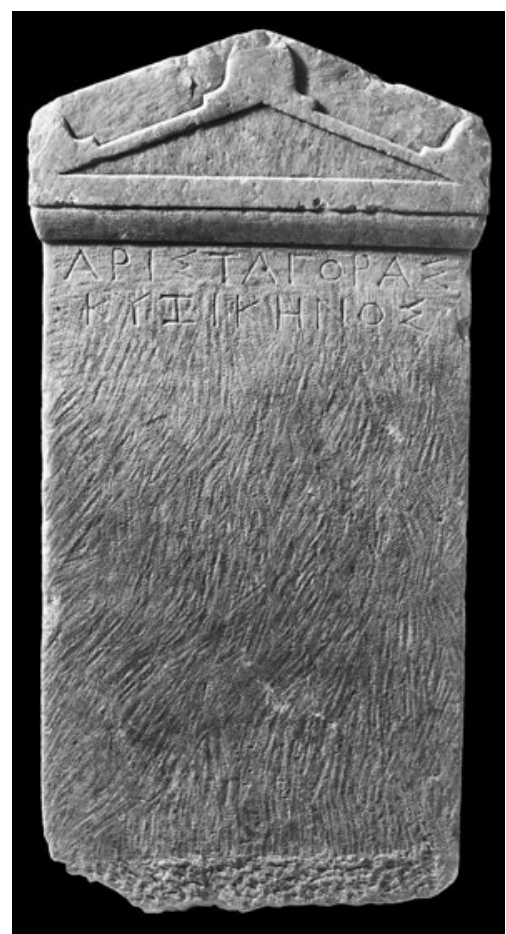

Fig. 45 - Stèle 29

(cl. ESAG, A. Skiadaressis). de I, nu un peu déséquilibré, omicron plus petit, sigma aux branches écartées.

$2^{\mathrm{e}}$ moitié du IV $\mathrm{e}$.

\section{i. 2. Analyse iconographique et stylistique de la stèle 1 (D. Ackermann)}

Les stèles funéraires érétriennes à relief figuré sont plutôt rares, d'où l'intérêt de cet exemplaire. Un beau parallèle, de même date, est fourni par la stèle d'Aristokratéia, trouvée dans la nécropole d'Alivéri/Porthmos et conservée au Musée d'Érétrie ${ }^{59}$. Toutes deux appartiennent au type bien connu des «Bildfeldstelen», c'est-à-dire des stèles de

59. ME 13062. C'est une stèle atticisante, voire attique vu la qualité de la réalisation du décor. Cf. A. Auberson, K. Schefold, Führer durch Eretria (1972), p. 178 (Schefold); D. Woysch-MÉAutis, La représentation des animaux et des êtres fabuleux sur les monuments funéraires grecs : de l'époque archä̈que à la fin du IV siècle av. J.-C. (1982), p. 118 n 172 pl. 26; A. Chatzidimitriou, dans C. Martin Pruvot et al. (n. 44), p. 303. 
petit format, le plus souvent ornées d'une représentation sculptée en relief dans un cadre creux, dont l'Attique a livré de très beaux exemplaires ${ }^{60}$. Si le marbre dans lequel notre stèle a été taillée semble local, l'iconographie de la scène et le style de la gravure orientent vers Athènes, et les meilleurs parallèles datent du $3^{\mathrm{e}}$ quart du Iv ${ }^{\mathrm{e}} \mathrm{s} .{ }^{61}$. On peut donc supposer que la stèle a été sculptée à Érétrie par un sculpteur athénien, ou par un sculpteur non athénien imitant les productions attiques. La réalisation quelque peu fruste du relief oriente peut-être vers la seconde hypothèse - bien que des stèles médiocres soient aussi produites en Attique ${ }^{62}$. Quoi qu'il en soit, l'art du relief funéraire sculpté attique a influencé nombre d'ateliers en Grèce à partir de la fin du v $v^{e}$ s., a fortiori dans les régions qui, comme l'Eubée, ne disposaient pas d'une tradition propre dans ce domaine. Par ailleurs, ce phénomène d'imitation est particulièrement présent dans les régions qui avoisinent l'Attique et qui sont politiquement influencées par Athènes, ce qui est le cas d'Érétrie pendant une bonne partie de l'époque classique ${ }^{63}$.

Le cadre du relief est architecturé : il comporte à gauche et à droite des antes dont seule la partie convexe du chapiteau a été représentée plastiquement; les détails devaient être peints. Sur le relief, gravé peu profondément, on voit une jeune femme aux cheveux courts, assise sur une chaise à dossier. Ses pieds nus sont sur un repose-pieds, la jambe droite en arrière, et la jambe gauche en avant. Elle est vêtue d'un chiton et d'un himation. Des deux mains, elle étreint celles d'une petite fille debout devant elle. Tête baissée, elle ne regarde pas l'enfant; elle a l'air pensif. L'enfant la regarde. Elle a les cheveux courts, est vêtue d'un chiton long et, par-dessus, d'un chiton plus court, qui descend jusqu'audessous du genou ${ }^{64}$. Elle semble tenir quelque chose dans la main droite, peut-être un oiseau.

60. Cf. A. Scholl, Die attischen Bildfeldstelen des 4. Jhs. v. Chr.: Untersuchungen zu den kleinformatigen Grabreliefs im spätklassischen Athen (1996) (ci-après Scholl).

61. E.g., pour le style et l'iconographie: Musée du Pirée $n^{\circ}$ 280, 340-330 (Scholl nº 310); Rhamnonte

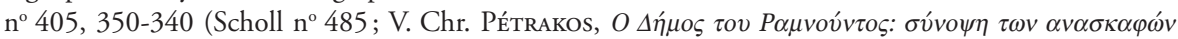

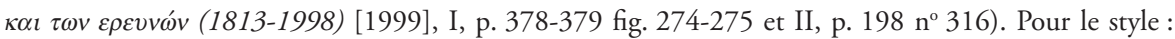
Athènes, Musée National no 997, 340-330 (Scholl n 136).

62. La graphie des idionymes, avec double tau, n'est d'aucun secours sur cette question; voir infra, p. 211.

63. Sur les stèles funéraires atticisantes, cf. Chr. W. Clairmont, Classical Attic Tombstones. Introductory Volume (1993), p. 73-80 (ci-après CAT). Sur les «Bildfeldstelen" atticisantes, cf. A. Scholl (n. 60), p. 82-84, qui observe que les ateliers situés hors de l'Attique ont pu être stimulés par une observation directe des stèles en Attique même ou par les quelques stèles attiques exportées (pour des exemples d'importations attiques à Érétrie, cf. CAT 1.826, 2.429a, 3.335). Clairmont évoque encore l'idée de «livres de modèles", mais leur existence est controversée.

64. Pour un parallèle, voir F. F. Jones, "An Athenian Stele in Princeton", dans Studies in Athenian Architecture, Sculpture and Topography Presented to Homer A. Thompson (1982), p. $63-64$ et pl. 10 (stèle attique du début du Iv e s.). Sur les vêtements féminins sur les stèles funéraires attiques classiques, cf. Chr. W. Clairmont (n. 63), p. 31-33. 
Les scènes représentant une femme assise avec un enfant debout devant elle ou se blottissant contre ses genoux sont fréquentes sur les stèles funéraires attiques depuis la reprise des reliefs sculptés dans la seconde moitié du ve s., et les «Bildfeldstelen" ne sont pas en reste ${ }^{65}$. Souvent, les deux figures interagissent en se tenant les mains ou par le biais d'un jouet ou d'un oiseau domestique. La scène illustre le profond attachement entre Thettalè et sa fille, attachement qui se prolonge au-delà du trépas puisque, réunies dans la mort, elles ont visiblement été enterrées au même endroit ${ }^{66}$.

\section{3. Considérations générales SUR La CHRONOLOGIE ET L'ONOMASTIQUe Des STÈLES (D. KNOEPFLER)}

En dépit de leur nombre limité par rapport à la totalité de la documentation épigraphique actuellement disponible (environ un millier de numéros) ou encore inédite (une cinquantaine au moins), ces quelque trois dizaines d'épitaphes peuvent donner une idée, certes incomplète, de la typologie et de l'onomastique des monuments funéraires érétriens aux $\mathrm{IV}^{\mathrm{e}}$ et III ${ }^{\mathrm{e}} \mathrm{s}$. C'est qu'elles forment un ensemble remarquablement homogène, aussi bien par leur provenance très précise (chose rare, on le sait, pour les inscriptions funéraires, le plus souvent remployées ou errantes) que par leur datation dans un espace

65. E.g. CAT 1.694 (420-400), 1.700 (400-375), 1.786 (400-375). «Bildfeldstelen»: voir le premier exemple donné supra, n. 61, auquel on peut ajouter Scholl n 516 (410-400) (=CAT 1.714. Baltimore, The Walters Art Gallery, 23.176) et 343 (360-350) (= CAT 1.871. Athènes, 3e Éphorie, M 1961), CAT 1.785 (360-330) (= R. Posamentir, Bemalte attische Grabstelen klassischer Zeit [2006], $\mathrm{n}^{\circ} 76$ et fig. 76.1. Musée du Pirée ${ }^{\circ}$ 260). Sur les stèles attiques d'époque classique représentant un enfant et sa mère, cf. O. Hirsch-Dyczeк, Les représentations des enfants sur les stèles funéraires attiques (1983), p. 39-44; H. RüHFel, Das Kind in der griechischen Kunst. Von der minoisch-mykenischen Zeit bis zum Hellenismus (1984), p. 142-165 (notamment p. 154 sur une stèle à nä̈skos attique du milieu du IV ${ }^{\mathrm{e}} \mathrm{s}$. exportée à Histiée-Oréos et représentant trois figures, dont une enfant se blottissant contre les jambes de sa mère, les deux figures s'étreignant les mains. Cette attitude n'est pas sans rappeler celle des deux figures de notre stèle. On en trouvera une illustration dans $A R 19$ [1972-1973], p. 8 fig. 14). Pour les «Bildfeldstelen », voir A. Scholl (n. 60), p. 124 et n. 841, qui donne plusieurs parallèles. Il relève que les mêmes scènes mais avec un homme à la place de la femme sont beaucoup plus rares. Voir ibid., p. 124 et n. 842 sur les «Bildfeldstelen» où un enfant vient se placer entre deux adultes engagés dans une dexiôsis, scène presque aussi courante que celle qui nous intéresse ici.

66. Nous n'avons aucune raison de douter que l'attachement des parents pour leurs enfants, y compris les filles, ait été bien réel et ne différait pas de nos standards actuels (cf. M. Th. Charlier, G. Raepsaet, "Étude d'un comportement social : les relations entre parents et enfants dans la société athénienne à l'époque classique», $A C 40$ [1971], p. 589-606). Contra J. MarCADÉ, "La mort des femmes, la mort des mères sur les stèles funéraires en Grèce à l'époque classique ( $\mathrm{v}^{\mathrm{e}}-\mathrm{IV}^{\mathrm{e}}$ siècle av. J.-C.) ", MMAI 93 (2014), p. 15 : «On aurait tort peut-être de surestimer la part de tendresse mêlée à la tristesse qu’introduit sur la stèle des femmes la présence d'enfants et de trop en juger selon notre sensibilité moderne : il semble plutôt que les enfants, sur les stèles funéraires féminines de l'époque classique, soient là pour compléter l'idée de la condition féminine par l'image de la maternité». 
de temps assez resserré, sans doute inférieur à deux siècles, dans la mesure où les indices paléographiques - combinés parfois avec d'autres critères - permettent d'établir une chronologie fiable.

\section{Alphabet et écriture}

Aucun des monuments, en effet, ne parait pouvoir être daté bien avant 400. L'absence de toute inscription en alphabet épichorique s'impose d'emblée comme un terminus post quem certes assez approximatif, mais d'une valeur néanmoins incontestable. C'était d'ailleurs déjà le cas, il faut s'empresser de le noter, dans la collection nettement plus abondante publiée par la regrettée Christiane Dunant en $1978^{67}$, comme aussi dans la série - plus disparate, assurément, à tous points de vue - réunie dix ans auparavant par Vassilios Pétrakos ${ }^{68}$. Les épitaphes archaïques, au sens le plus large du terme, restent d'ailleurs, aujourd'hui encore, très rares à Érétrie : dans le corpus de 1915, il n'y en a pratiquement aucune qui soit gravée dans l'alphabet épichorique de l'Eubée centrale (et de la Béotie voisine $)^{69}$. C'est pourquoi il nous paraît utile de faire connaître ici - ne serait-ce que sommairement - une assez monumentale stèle de marbre (attique?) restée inédite $^{70}$ (quoique apportée au Musée d'Érétrie voici plusieurs lustres déjà) portant le

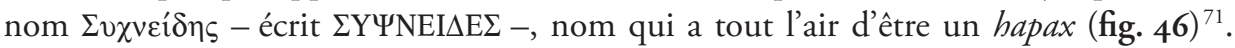

67. Chr. Dunant (n. 55) (188 numéros; mais cf. infra, n. 190).

68. AD 23 (1968), Mel., p. 99-116 et pl. 46-49, avec un complément ibid. 29 (1974), Mel., p. 100-108 et pl. 58-73 (env. 120 numéros au total, mais beaucoup à l'état de petits fragments; pour le $\mathrm{n}^{\circ} 88$, voir la note suivante).

69. Voir IG XII 9, 285-288 (épigrammes funéraires) + 296-302 ("tituli antiquissimi»). Les épigrammes ont été rééditées dans le recueil de P. Hansen, Carmina Epigraphica Graeca (ci-après CEG) I (1983), $\mathrm{n}^{\text {os }}$ 76-77 (rangées - un peu abusivement, nous semble-t-il - avec celles d'Athènes en raison de l'usage qui y est fait de l'alphabet attique) et 106-108 (pour le n 107 , un des très rares documents funéraires gravés dans l'alphabet épichorique, cf. infra, n. 113). Concernant la borne funéraire IG XII 9, 302, HOPO $\mathrm{\Theta HKH \Sigma}$, on notera qu'elle ne fait sans doute qu'un avec le fragment publié par V. PÉTRAKOS (n. 68 [1968]), nº 88 (cf. Bull. ép. 2011, 313, en p. 331). Pour l'épitaphe dialectale IG XII 624, voir ci-après p. 206 avec la n. 104.

70. Musée d'Érétrie, sans numéro d'inventaire. La photo reproduite ici avec l'aimable autorisation de l'Éphorie de l'Eubée date de 1977 (une autre avait été prise par l'auteur dès 1973). L'entrée de la pierre au Musée doit se situer vers 1972, puisque cette inscription non inventoriée ne figure pas dans le supplément épigraphique publié par V. PéTrakos (n. 68 [1974]).

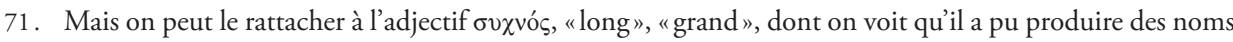

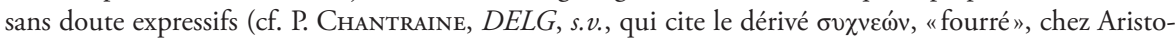
phane). Pour les composés en - $\varepsilon 1 \delta \eta \varsigma$ (type 'Y $\pi \varepsilon \rho-\varepsilon i ́ \delta \eta \varsigma)$, voir Fr. Bechtel, Die Historischen Personennamen des Griechischen bis zur Kaiserzeit (ci-après HPN) (1917), p. 149 : on peut ajouter à sa liste un

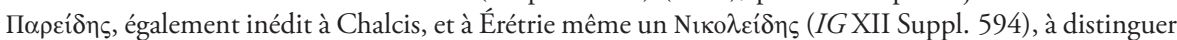

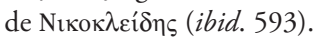


Cette stèle de haute taille $\mathrm{e}^{72}$ présente d'ailleurs en son sommet une moulure ou cimaise très semblable à celle du monument probablement le plus ancien de la série publiée ici $(\mathbf{3})^{73}$. Celui-ci témoigne déjà, assurément, de l'adoption de l'alphabet ionien-attique, mais présente encore, en revanche, un chi en croix de type attique ${ }^{74}$ et non pas déjà ionien $(\mathrm{X})$ comme on aurait pu l'attendre à cette date. De fait, l'abandon de l'alphabet épichorique a dû se produire en Eubée - ou en tout cas à Érétrie - à une époque où l'influence politique et culturelle de l'Attique était plus forte que celle de la Béotie voisine, donc encore avant le tournant de 411. Ce que confirme, au demeurant, l'alphabet utilisé dans le plus ancien décret érétrien de proxénie connu à ce jour, datable des alentours de 450-425, tandis qu'au lendemain de la libération de 411 c'est déjà l'alphabet ionien (ou ionien-attique) qui est la norme pour les documents publics ${ }^{75}$.

Le support des inscriptions: les types représentés et les types absents

Comme le catalogue l'a bien mis en évidence,

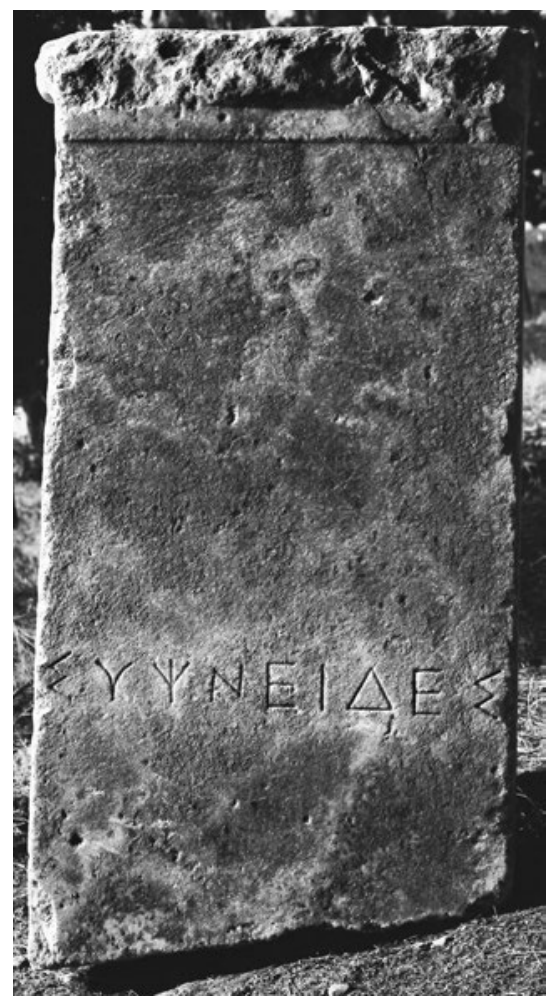

Fig. 46 - Stèle de $\Sigma v \chi v \varepsilon i ́ \delta \eta\rceil$ (cl. ESAG, D. Knoepfler). la plupart des stèles appartiennent à des types fort simples connus et répertoriés depuis très longtemps, puisqu'une typologie des épitaphes érétriennes avait été établie dès la fin $\mathrm{du} \mathrm{XIX}^{e}$ s. par l'archéologue danois

72. Dimensions de cette stèle légèrement pyramidante, avec un couronnement mouluré : $0,97 \mathrm{~m}$ de hauteur (moulure 0,115 m) ; 47,7 m (en haut)-49,5 m (en bas) de largeur; 0,12 m d'épaisseur.

73. Pour un couronnement de même type, voir aussi $I G$ XII 9, 457 et 505, datés sans doute trop tardivement du III $^{\mathrm{e}}$ s. seulement. Pour l'emplacement du nom, cf. IG XII 9, 335 (vers 400).

74. Voir L. H. Jefferr, The Local Scripts of Archaic Greece ${ }^{2}$ (1992), p. 66 (Attique); cf. aussi p. 79 pour son usage en Eubée au v $\mathrm{v}^{\mathrm{e}}$.

75. Cf. D. Knoepfler, Décrets érétriens de proxénie et de citoyenneté, Eretria XI (2001), p. 69-76 nº I, avec la fig. 10B. Dans le décret $\mathrm{n}^{\circ}$ II, qui date de 411, la graphie attique ПPO+ $\mathrm{ENON}$ du $\mathrm{n}^{\circ} \mathrm{I}$ a déjà fait place à la graphie ionienne ПРOछENON (ibid., p. 77, $\mathrm{n}^{\text {os }}$ II-III). 
Christian Blinkenberg ${ }^{76}$. À cet égard il n'y a donc que peu de surprises. Mais une différence (peut-être fortuite) - et de nature typologique bien plutôt que chronologique - apparaît entre le lot des stèles du Torrent et celui des abords de la Porte de l'Ouest publié par Chr. Dunant : c'est l'absence, dans le premier, d'un type de monuments funéraires qui, à Athènes et en Attique, a connu un développement significatif à partir de la fin du $v^{e} s$., à savoir celui des niches funéraires surmontées le plus souvent d'un fronton, mais pouvant aussi être couvertes par un linteau plat imitant le rebord d'un toit, avec une ou plusieurs statues se détachant en haut-relief sur la dalle arrière (alors que le relief, dans la stèle publiée ici sous le numéro 1, est taillé à l'intérieur d'un simple cadre creux ménagé dans la surface du fût). Chose notable, en effet, parmi les stèles recueillies par les archéologues suisses à la fin des années 1960 devant le rempart occidental se trouvaient deux épistyles inscrits appartenant manifestement à ce type de monuments à naïskos et datant, selon toute probabilité, du $\mathrm{IV}^{\mathrm{e}} \mathrm{s} \cdot{ }^{77}$; un troisième spécimen, de date voisine, était remployé comme seuil dans la phase hellénistique de l'Édifice I ${ }^{78}$, tout proche de ce rempart. D'autres épistyles de même type restent à publier : l'un, repéré dès 1974 par la regrettée Ingrid Metzger à l'Ouest du cimetière moderne, provient certainement, lui aussi, de la nécropole occidentale, où cette niche de grandes dimensions devait se dresser en bordure immédiate de la route menant vers $\mathrm{Chalcis}^{79}$; un autre se trouve remployé, avec un nombre important de stèles funéraires, dans le secteur des vestiges paléochrétiens et byzantins d'Hagia Paraskevi, à l'Est de la ville antique ${ }^{80}$. L'absence, dans le nouveau lot, de tout fragment de naïskos pourrait donc ne pas être dû au hasard. En Attique, d'ailleurs, ces monuments côtoient souvent les stèles à haut fût, décorées de deux rosettes et surmontées d'un anthémion plus ou moins développé; or, ce type de stèles non plus n'est pas représenté dans la série de 2009, tandis qu'il y en a au moins un spécimen mutilé dans le recueil de $1978^{81}$, sans parler des nombreux exemples de

76. Eretriske Gravskrifter (1891) (avec un résumé en français), qui distinguait quatre types principaux de supports. On ne connaissait alors ni stèles à anthémion ni monuments en forme de naïskos.

77. Chr. Dunant (n. 55), n no 106 et 107, avec la pl. 21. Cf. déjà $I G$ XII 9, 699.

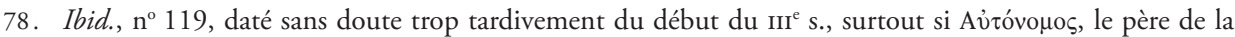
défunte, devait être identifié avec l'amiral érétrien homonyme honoré à Delphes dans le «Monument des Navarques» : cf. LGPN I, s.v. $\mathrm{n}^{\text {os }} 5-6$, où cette identification a été proposée par le soussigné.

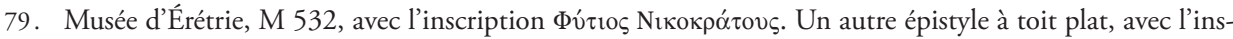

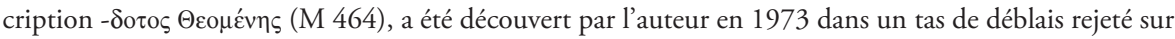
un terrain public au Sud-Est de la ville moderne.

80. Repéré par le soussigné dans la construction d'une tombe paléochrétienne située au Nord-Est de cet

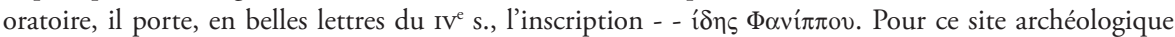
(dont la fouille déjà ancienne a été complétée en 2007), cf. Bull. ép. 2012, 231 et 2015, 346.

81. Chr. Dunant (n. 55), n 162 et pl. 31. Ce fragment, datable des alentours de 300 sur la base de la gravure, avait été trouvé dans la batterie de tir de la Porte de l'Ouest. 
grande qualité qu’abrite le Musée d'Érétrie ${ }^{82}$. Il semble ainsi permis d'en déduire que le secteur touché par la récente crue du torrent, se trouvant déjà un peu à l'écart des voies les plus fréquentées au sortir de la ville, ne comportait sans doute qu'un nombre réduit de grands tertres familiaux ${ }^{83}$ de l'espèce de ceux que fait connaitre la publication des nécropoles de Rhamnonte : c'est, en effet, au sommet de ces périboloi somptueusement édifiés que se dressaient, souvent côte à côte, ces deux types de monuments raffinés ${ }^{84}$. D'autres secteurs plus favorablement situés de la vaste nécropole érétrienne, tant sur le flanc occidental que, surtout, peut-être, du côté oriental ${ }^{85}$, devaient offrir au regard des passants de semblables enclos funéraires; et cela dès le début du IV ${ }^{e}$ s., époque où certaines familles de la ville - celles en particulier qui vivaient dans les luxueuses maisons mises au jour par les fouilles suisses au pied de l'Acropole - purent, à coup sûr, faire la dépense de tombeaux assez somptueux.

\section{Chronologie : la limite basse}

$\mathrm{Si}$, après cet excursus, on en revient à l'examen des stèles publiées ici, on constate que la fourchette chronologique dans laquelle elles se renferment n'est pas moins bien fixée vers le bas que vers le haut. En effet, aucune ne saurait être datée de la période hellénistique avancée $\left(\mathrm{II}^{\mathrm{e}}-\mathrm{I}^{\mathrm{er}} \mathrm{s}\right.$.) et mise en relation avec la seule tombe de cette époque qui ait été repérée dans le secteur d'où proviennent nos inscriptions (T16). Quelques-unes, assurément - ainsi le numéro $\mathbf{2 4}$ - offrent des particularités d'écriture qui peuvent inciter à les mettre vers le milieu - voire déjà vers la fin? - du III ${ }^{\mathrm{e}}$ s. plutôt qu'aux alentours de 300 (comme c'est le cas de la majorité d'entre elles). Mais il n'y en a aucune, marquons-le bien ici, dont les particularités graphiques doivent faire penser à une phase plus évoluée de l'écriture lapidaire; pas de trace d'alpha à barre brisée, pas de thêta à barre horizontale en lieu et place du point, pas même de sigma aux branches totalement horizontales (même si l'on se rapproche de cette forme sur la stèle 24); pas non plus d'exemple d'inscription

82. Voir A. Auberson, K. Schefold (n. 59), p. 166 et 175-178, pour celles qui étaient exposées alors au

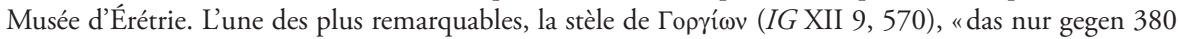
gefertigt sein kann" (loc. cit.), est également intéressante en ce qu'elle montre qu'au début du IV s., certains des monuments les plus élaborés donnaient déjà le patronyme du défunt.

83. Deux terrasses funéraires ont cependant été repérées parmi les tombes que cette crue a mises au jour : cf. supra, p. 160-162.

84. Voir V. PÉtrakos (n. 61), I, p. 335-413, avec les nombreuses reconstitutions de tels périboles, qui montrent en bien des cas l'association des monuments en naïskos avec les stèles à rosettes et, parfois, à anthémion. Pour Athènes même, voir D. Marchiandi, I peroboli funerari nell'Attica classica : lo specchio di una "borghesia" (2011).

85. Pour ces grands monuments de la nécropole orientale - dont la trop fameuse "Tombe d'Aristote» - voir St. G. Schmid, "Ein Ungeheuer in Eretria», MH 55 (1998), p. 193-211, en particulier p. 208-211 sur l'extension de cette nécropole en direction de Kato-Vathia/Amarynthos. 
dont les lettres anguleuses seraient déjà pourvues d'apices caractérisés ${ }^{86}$. Car la présence d'extrémités plus ou moins appuyées est un trait de style qui apparait dès la fin $\mathrm{du}_{\mathrm{IV}}^{\mathrm{e}} \mathrm{s}$.

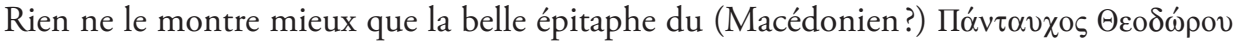
exposée au Musée d'Érétrie : cette stèle que son décor en relief (deux boucs affrontés de part et d'autre d'un cratère dionysiaque) permet de dater avec confiance du dernier quart du IV $v^{e} .^{87}$ fait voir des lettres de ce type gravées avec le plus grand soin.

Pour ce qui est du libellé, il faut noter l'absence significative de toute stèle portant la formule $\chi \rho \eta \sigma \tau o ́ \varsigma / \chi \rho \eta \sigma \tau \eta^{88}$, avec ou sans $\chi \alpha i ̂ \varepsilon^{89}$, car le lot bien plus considérable publié en 1978 offre sur ce point un faciès un peu différent : en effet, on y recense au moins trois stèles datant des alentours de 300 où le nom d'une femme sans patronyme - absence qui, à l'époque encore haute des monuments en question, est probablement sans rapport avec le statut des défuntes - est suivi de cet adjectif (sans la formule de salut), à quoi s'ajoute un quatrième exemple, plus tardif certes (mais qui pourrait néanmoins encore remonter à l'extrême fin du III $^{\mathrm{e}}$ s.) pour une femme de condition libre, si l'on en juge par la présence d'un patronyme ${ }^{90}$. De fait, tout indique que l'ajout de cette épithète (quelle qu'en ait été, à l'origine, la signification précise) n'est pas exceptionnel, pour les femmes en particulier, dès la haute époque hellénistique, même si la formule complète ne fait irruption, elle, qu'à date tardive dans l'épigraphie funéraire d'Érétrie (guère avant le milieu du II ${ }^{\mathrm{e}} \mathrm{s}$.) pour devenir banale à partir du siècle suivant ${ }^{91}$. L'absence d'une épitaphe de ce type dans le nouvel ensemble est donc tout à la fois fortuite et normale.

86. Des apices encore légers, d'un type très courant au $\mathrm{III}^{\mathrm{e}} \mathrm{s}$. déjà, s'observent ainsi dans le $\mathrm{n}^{\circ} \mathbf{2 4}$. Chr. DunANT (n. 55) notait déjà que, sur un certain nombre de stèles provenant des abords de la Porte de l'Ouest, «les extrémités des lettres sont plus appuyées, annonçant l'apparition des apices des siècles ultérieurs» (p. 25).

87. IGXII 9, 715; cf. A. Auberson, K. Schefold (n. 59), p. 176 (datation), avec la bibliographie en n. 183 (p. 203); depuis, ajouter D. KNoepfler, "Autour d'une stèle "mégarienne" ", dans P. Ducrey (éd.), Mélanges d'histoire ancienne et d'archéologie offerts à Paul Collart (1976), p. 269-276, avec une photo en fig. 2.

88. On sait que cet adjectif ne doit plus être considéré - sauf preuve du contraire - comme l'indice d'un statut servile : voir notamment L. RoBert, Hellenica VII (1947), p. 152.

89. La présence de cette salutation marque une étape certainement plus avancée dans l'évolution. Dans IG XII 9, toutes les épitaphes érétriennes dotées de cet adjectif ont été groupées sous les $\mathrm{n}^{\text {os }} 862-889$, qu'elles aient ou non la salutation.

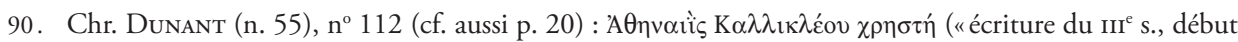
d'apices").

91. Pour la Béotie, cf. P. M. Fraser, T. Rönne, Boeotian and West Greek Tombstones (1957), p. 92. Dans la série d'épitaphes hellénistiques de Thèbes - dont la majorité remonte aux $\mathrm{III}^{\mathrm{e}}$ et $\mathrm{II}^{\mathrm{e}} \mathrm{s}$. - récemment publiée par M. Bonnano-Aravantinou, dans N. Papazarkadas (éd.), The Epigraphy and History of Boeotia. New Finds, New Prospects (2014), il n'y en a qu'une seule avec l'adjectif $\chi \rho \eta \sigma \tau \eta ́$, et c'est l'une des plus tardives (p. $304 \mathrm{n}^{\circ}$ 2). Cf. aussi Y. Kalliontzis, qui propose d'attribuer à Érétrie une stèle du Musée de Thèbes

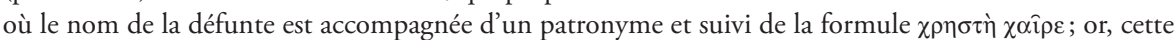

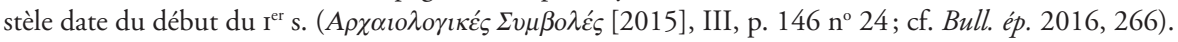


De ces divers indices chronologiques il paraît ainsi légitime d'inférer que ce secteur au moins de la nécropole occidentale fut délaissé dès avant le début du $\mathrm{II}^{\mathrm{e}}$ s., soit parce qu'il n'offrait plus de place pour de nouvelles sépultures, soit, bien plutôt, parce qu'il avait été ruiné - ou rendu peut-être inaccessible - en une circonstance précise, puis abandonné et enfoui par les apports du torrent saisonnier ${ }^{92}$. On est ainsi tenté de penser à un événement "violent, cataclysme naturel ou fait de guerre", comme l'écrivait déjà très justement Chr. Dunant ${ }^{93}$, en constatant que la plupart des stèles publiées par elles (et datant, comme les nôtres, des $\mathrm{IV}^{\mathrm{e}}$ et $\mathrm{III}^{\mathrm{e}}$ s.) avaient été remployées en une seule fois dans la couverture d'une canalisation hellénistique et dans la reconstruction de la maison voisine (Édifice I) et que beaucoup d'autres aussi gisaient encore dans le canal séparant le rempart de la nécropole. Ce tournant historique a les meilleures chances de correspondre au siège de la ville par les Romains et leurs alliés en l'an $198^{94}$, car la proximité de ce secteur avec les ouvrages défensifs que l'inondation de 2009 a remis brutalement au jour pourrait rendre compte aisément du sort fait à ces tombes. La seule différence - mais notable - entre les deux ensembles tiendrait donc au fait qu'en raison de leur éloignement relatif par rapport à l'habitat, nos stèles auraient été laissées sur place, tandis qu'au contraire celles qui se dressaient en grand nombre, avant 198, dans la nécropole située au Sud de la Porte de l'Ouest furent massivement réutilisées dans les travaux de remise en état des édifices endommagés par le siège.

\section{L'introduction du patronyme dans la formule onomastique}

Au sein de l'ensemble de 29 numéros publiés ici, les deux tiers des épitaphes ne comportent que le nom du défunt ou de la défunte (2-19), tandis qu'un tiers seulement est constitué de stèles présentant nom et patronyme ( $\mathbf{1}^{95}$ et 20-29). Compte tenu de l'époque, cette répartition n'a rien de surprenant, puisque l'on retrouve exactement la même proportion dans la série, pourtant bien plus abondante, publiée en 1978. En effet, Chr. Dunant relevait 111 noms seuls contre 53 seulement avec patronyme, en notant que la statistique menée précédemment par P. M. Fraser et T. Rönne ${ }^{96}$, dans le

92. Voir ci-dessus, p. 145-146, les observations faites lors de l'exploration archéologique de ce secteur.

93. Chr. Dunant (n. 55), p. 25, qui rappelle en n. 23 que la mise en relation de ce constat archéologique avec le siège de 198 fut opérée "par K. Schefold dès son premier rapport sur les recherches de la Mission suisse à Érétrie, $A K 7,1964,104$ »; cf. A. Auberson, K. Schefold (n. 59), p. 25 et 87-88. Bibliographie complémentaire chez K. ReBER, Die klassischen und hellenistischen Wohnhäuser im Westquartier, Eretria X (1998), p. 25 n. 61 ; pour le remploi de 23 stèles dans la phase 3 de l'Édifice I - sans compter celles, beaucoup plus nombreuses, ayant servi à la couverture de la canalisation voisine - voir ibid., p. 60-62, avec la discussion sur le terminus post quem.

94. Voir l'introduction par D. Ackermann, p. 171.

95. Pour cette stèle hors norme, voir ci-après p. 206 et 211.

96. P. M. Fraser, T. RönNe (n. 91), p. 98-100; sur la question du démotique, cf. infra, p. 206. 
cadre de leur étude sur l'épigraphie funéraire béotienne, aboutissait à un résultat assez comparable - mais néanmoins un peu différent, ajouterons-nous, puisque sur les 670 épitaphes érétriennes alors connues de ces auteurs à travers la collection des $I G$, il s'en trouvait 270 environ avec le nom seul pour environ 330 avec le patronyme. Or, cette inversion du rapport entre les deux catégories s'explique très largement par le fait que sont prises en compte, dans ce recueil, d'assez nombreuses inscriptions datant de la basse époque hellénistique et de l'époque romaine, quand la mention du patronyme se fut généralisée en Eubée (tandis que la Béotie demeurait fidèle à l'usage ancien). Cela peut rendre compte également des chiffres - allégués aussi par l'épigraphiste genevoise - que fournit la publication d'un peu plus de 100 stèles (dont plusieurs certes peu utilisables pour une statistique de ce genre) publiées par V. Pétrakos ${ }^{97}$ (soit 37 avec le nom seul et 39 avec le patronyme), car il s'agit là d'un ensemble disparate tant au point de vue chronologique que, d'abord, sur le plan des provenances. Il convient donc de s'en tenir à la comparaison, suffisamment instructive, avec les stèles recueillies dans les premières fouilles suisses, en laissant de côté "les totaux étonnamment équilibrés ${ }^{98}$ obtenus par l'addition de toutes ces données (soit 434 noms seuls et 426 noms avec patronyme), car cet équilibre apparent a conduit l'éditrice à soutenir, conformément du reste à l'opinion déjà exprimée par P. M. Fraser et T. Rönne, que «la présence ou l'absence du patronyme était à Érétrie une affaire de choix personnel» (sans rapport apparent avec le statut, ni non plus avec la chronologie).

Sans contester, certes, que les proches du défunt aient pu, à toute époque, faire librement le choix d'une des deux possibilités, on ne saurait éliminer le facteur chronologique, qui permet de voir dans quel sens va l'évolution. Du reste, l'éditrice avait bien voulu faire état d'une observation que nous lui avions adressée à ce propos, en notant "qu’il conviendrait de tenir compte de la date des stèles publiées ici, qui appartiennent précisément à la période de transition entre l'usage du nom seul et celui du nom avec patronyme, qui tend à s'imposer dès le III $^{\mathrm{e}}$ siècle " ${ }^{99}$. Ce qui permet de l'établir sur une base solide, en effet, c'est la pratique observable dans les documents publics érétriens, en particulier les décrets, où l'on observe que les noms des auteurs de proposition peuvent être assortis du patronyme dès les premières attestations de cette formule vers 340, mais que l'usage ancien, sans patronyme, se rencontre jusqu'à la fin $\mathrm{du} \mathrm{IV}^{\mathrm{e}} \mathrm{s} .{ }^{100}$. Il est vrai que la nomenclature officielle complète, avec ajout du démotique, n’a jamais réussi à

97. V. Pétrakos (n. 68 [1968]), avec son supplément publié en 1974 dans ce même périodique, dont Chr. Dunant ne paraît pas avoir eu connaissance. Les chiffres donnés ci-dessus tiennent compte des quelque 20 numéros du supplément.

98. Chr. Dunant (n. 55), p. 24.

99. Ibid.

100. Voir D. Knoepfler (n. 75), p. 309-310 (cf. aussi p. 72 et n. 277 pour l'usage dans les stèles funéraires). La publication par le soussigné, REA 119 (2017) p. 395-484, de trois nouveaux décrets ne fait que 
se généraliser dans l'épigraphie privée, où les cas d'usage d'un démotique sont demeurés très sporadiques ${ }^{101}$. Dans la grande série des stèles publiée en 1978, il n'y en a qu'un tout petit nombre d'exemples ${ }^{102}$ : c'est donc sans surprise que l'on constate l'absence de toute stèle avec démotique dans le nouveau lot. Pour ce qui est du patronyme, en revanche, on peut admettre que la tendance à l'ajouter dans les épitaphes aussi a été forte dès avant la fin du $\mathrm{IV}^{\mathrm{e}}$ s. Les 18 stèles qui en sont encore dépourvues ne contredisent pas cette affirmation, puisque - à l'exception, peut-être, des numéros 5 et 7 - elles peuvent toutes remonter, de manière plus ou moins probable, au Iv ${ }^{\mathrm{e}} \mathrm{s}$. À cette époque, l'usage du patronyme n'était pas encore entré complètement dans les mœurs, comme en témoigne la stèle à relief de type attique publiée ici même sous le numéro 1 : bien que la mère et la

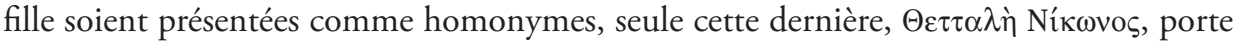
un patronyme, dont la présence a dû être ressentie comme d'autant plus nécessaire que ce père de la fillette était en même temps, peut-on penser, l'époux de la mère.

L'absence de patronyme ne saurait certes garantir l'ancienneté d'un monument, si d'autres arguments - de nature paléographique ou typologique - viennent plaider pour une date plus récente : l'exemple de la Béotie voisine prouve assez, en effet, que la fidélité à l'usage ancien a pu être tenace en certains pays ou milieux ${ }^{103}$. Inversement, un patronyme peut se rencontrer dès la première moitié $\mathrm{du}_{\mathrm{IV}}^{\mathrm{e}} \mathrm{s}$. : rien ne le montre mieux que l'inscription (rédigée encore en dialecte, mais gravée déjà dans l'alphabet ionien-

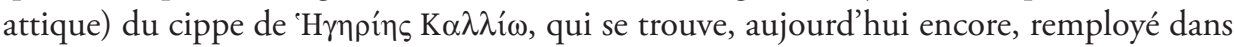
un mur tardif du Gymnase ${ }^{104}$. La présence d'une forme dialectale constitue, en principe, un critère chronologique de grande valeur (sans être toujours décisif pour autant). Dans

confirmer cette conclusion, qui est d'ailleurs conforme à ce que l'on peut observer dans d'autres cités, ainsi à Athènes (après 353/2) et à Samos (dès 322/1).

101. Mais on ne peut plus dire que «none has the demotic, although Eretria had a fully developped demesystem» (P. M. Fraser, T. Rönne [n. 91], n. 25 p. 100).

102. Chr. Dunant (n. 55), n 161, stèle familiale, où, comme le rappelle l'éditrice, nous avons pu retrouver le démotique [P $\alpha \varphi \imath \varepsilon \hat{]}] \theta \varepsilon v$ en raison de l'onomastique (dans l'index, il faut de toute façon supprimer le

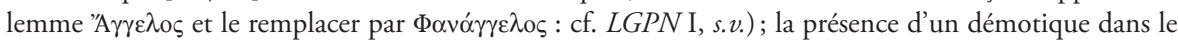
$\mathrm{n}^{\circ} 136$ est moins bien assurée; et elle est tout à fait douteuse dans le $\mathrm{n}^{\circ} 107$ (cf. SEG LI 1120 ter, pour une opinion qui, depuis, a cessé d'être la nôtre). Dans le corpus, l'unique épitaphe à livrer un démotique est la stèle $I G$ XII 9, 532; un autre cas très probable se trouve sur une stèle provenant de la nécropole de Rhamnonte : cf. D. KNoepfler (n. 75), p. 240-241 et fig. 50.

103. Pour la Béotie, outre l'ouvrage déjà cité de P. M. Fraser, T. Rönne (n. 91), voir les publications récentes de Y. Kalliontzis, en particulier Grammateion 3 (2014), p. 15-30, pour une série d'épitaphes orchoméniennes (cf. Bull. ép. 2015, 279). À Oropos, en revanche, sous l'influence conjuguée d'Athènes et d'Érétrie, l'usage du patronyme dans les épitaphes est très couramment attesté dès le début du III $^{\mathrm{e}} s$.

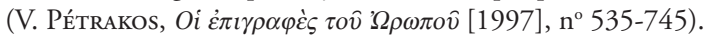

104. IG XII 9 624. Photo chez E. Mango, Das Gymnasion, Eretria XIV (2003), p. 62 fig. 70 (cf. 150 E 24). Pour l'analyse des formes dialectales, voir M. L. DEL BARRIO VEGA, L'Eubée (Paradeigmata : recueil d'inscriptions grecques dialectales, II 2) (2015), p. 89-90 EUB 88 (cette épitaphe ne saurait être postérieure au 
la série publiée ici, de tels cas sont bien peu nombreux, puisque l'on ne trouve guère

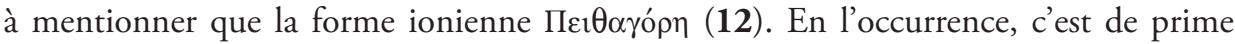
abord un signe d'ancienneté d'autant plus probant que le nom (nouveau en Eubée ${ }^{105}$ ) n'est pas accompagné d'un patronyme et que le support lui-même, une simple stèle à bandeau lisse, est très caractéristique $\mathrm{du}_{\mathrm{IV}}^{\mathrm{e}} \mathrm{s}$. Le flottement dialectal dans la désinence de tels noms est cependant exemplifié par la stèle 8, qui paraît être au moins aussi

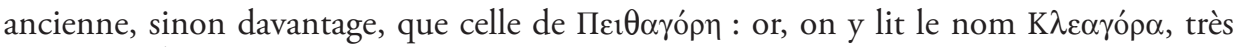
typique d'Érétrie, où il était déjà connu sous la forme $\mathrm{K} \lambda \varepsilon \alpha \gamma o ́ p \eta$ par au moins deux autres exemples, dont le plus récent, gravé dans une écriture du $\mathrm{III}^{\mathrm{e}}$ s. $^{106}$, appartient à une époque où le dialecte ionien d'Érétrie - en recul devant la koinè dès les années 330$320^{107}$ - avait pratiquement disparu. Une attestation à coup sûr ancienne, en revanche, de l'élément - $\alpha \gamma o ́ p \eta$ dans l'onomastique érétrienne est fournie par la dédicace à Ilithyie

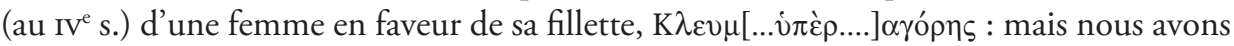
pu montrer naguère, dans le sillage de G. Daux, qu'en dépit de la gravure stoichèdon de cette consécration monumentale provenant de la palestre, la restitution $[\mathrm{K} \lambda \varepsilon] \alpha \gamma o ́ \rho \eta \varsigma, ~ s i$ vraisemblable soit-elle (compte tenu de la popularité, dans l'élite sociale de cette cité, de l'anthroponyme féminin en question), n'y était pas assurée, d'autres noms de même

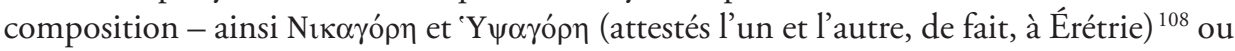

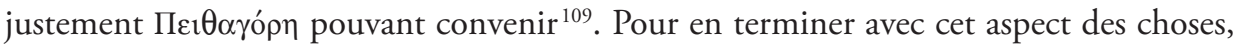
notons encore que si nos stèles n'offrent aucun exemple du fameux rhotacisme érétrien - qui perdura pourtant bien après 300 dans un certain nombre d'anthroponymes locaux

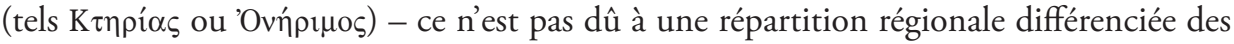
anthroponymes rhotacisés, comme on avait pu en émettre autrefois l'hypothèse ${ }^{110}$, mais tout simplement au fait que, dans le lot des stèles provenant du torrent, il n'y a guère que

milieu du $\mathrm{IV}^{\mathrm{e}}$ s.). Un autre exemple de patronyme pourvu d'une désinence dialectale est fourni par une

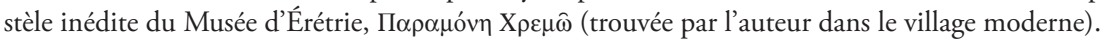

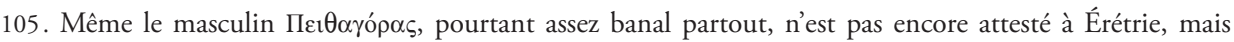
seulement, pour l'Eubée, à Chalcis (LGPN I, s.v.).

106. IG XII 9, 303 et 624 (avec patronyme). Cf. LGPNI, s.v. nos 2-3. Un autre exemple de K $\lambda \varepsilon \alpha \gamma o ́ p \alpha$ à Érétrie est en revanche des plus incertains, tant pour le radical que pour la désinence : voir Chr. Dunant (n. 55), $\mathrm{n}^{\circ} 103$ ( $L G P N \mathrm{I}$, s.v. $\mathrm{n}^{\circ}$ 1). Il est notable que la seule $\mathrm{K} \lambda \varepsilon \alpha \gamma o ́ \rho \alpha$ attestée en Béotie vienne d'un inventaire hellénistique d'Oropos (V. Pétrakos, I. Oropos 323, 1. 24; cf. LGPN III.B, s.v. n 1 ).

107. Voir D. Knoepfler (n. 75), p. 107-108 et 181.

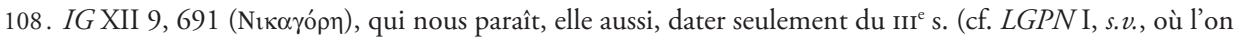

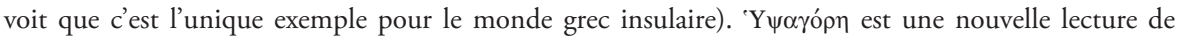

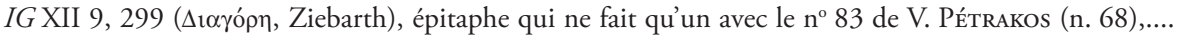
$\mathrm{OPH}$ : cf. $L G P N \mathrm{I}$, s.v.

109. La longueur du nom de la dédicante ne peut pas, en effet, être exactement déterminée : voir D. KNOEPfLer, "Dédicaces érétriennes à Ilithyie», $A K 33$ (1990), p. 115-128, avec la fig. 2 et la pl. 4-5.

110. J. D. Рнокітіs, Le rhotacisme dit érétrien (1932). Contre cette hypothèse, voir la bibliographie réunie par D. Knoepfler, "Anthroponymie et géographie régionales: le cas des dèmes d’Érétrie», dans 
le nom de la stèle $25, \Lambda v \sigma i ́ \mu \alpha \chi 0 \varsigma^{111}$, qui soit susceptible d'être affecté par ce phénomène phonétique actif jusque vers la fin du $\mathrm{IV}^{\mathrm{e}} \mathrm{s} .{ }^{12}$.

\section{Hommes et femmes dans la nécropole}

Pour ce qui est de la répartition des épitaphes entre les deux sexes, on y compte quinze femmes pour treize hommes (sur la stèle $\mathbf{2 3}$, dont n'est conservée que la partie gauche, on ne peut pas déterminer le sexe du défunt). Cette proportion ne varie pas significativement d'une catégorie à l'autre, le nombre des femmes restant légèrement supérieur à celui des hommes même après que le patronyme a été, le plus souvent, ajouté à l'idionyme. La différence est d'ailleurs exactement du même ordre dans la série très homogène publiée par Chr. Dunant ( 82 femmes pour 69 hommes), tandis qu'au contraire la collection passablement hétérogène réunie par V. Pétrakos offre une proportion inversée, mais de peu (30 femmes pour 33 hommes). La prise en compte de l'ensemble du corpus montrerait que, d'une façon générale, le nombre des inscriptions funéraires est légèrement plus élevé - à Érétrie comme en bien d'autres cités - pour les femmes que pour les hommes. On peut en rendre compte, au moins en partie, par le fait que les hommes tombés au service de la cité pouvaient être inhumés dans des tombeaux collectifs construits aux frais de l'État - ces polyandreia dont le nom lui-même atteste qu'ils ne concernaient que l'élément masculin de la population -, avec des stèles fournissant les noms de ces soldats ${ }^{113}$. Cela paraît prouver en tout cas que, dans les milieux où l'on pouvait songer à élever un monument aux membres défunts de la famille, il y avait, sur ce plan, une stricte égalité entre les sexes. Aucune différence non plus ne paraît avoir existé - comme il ressort d'une statistique établie par Chr. Dunant sous la forme d'un tableau ${ }^{114}$ - sur le plan typologique, c'est-à-dire dans le choix du monument lui-même, entre les divers types de stèles offerts par les tailleurs de pierre et les lapicides à leur clientèle en cette phase de l'histoire érétrienne. Et il est à peine besoin d'ajouter

Cl. Balandier, Chr. Chandezon (éds), Institutions, sociétés et cultes de la Méditerranée antique. Mélanges d'histoire ancienne rassemblés en l'honneur de Claude Vial (2013), p. 55.

111. De fait, la forme $\Lambda$ pí $\mu \alpha \chi 0 \varsigma$ est attestée vers la fin du Iv s., cf. $L G P N \mathrm{I}$, s.v. $\mathrm{n}^{\circ} 1$.

112. Si plusieurs anthroponymes à sigma intervocalique non rhotacisé figurent dans le lot publié en 1978 (voir Chr. Dunant [n. 55], $\mathrm{n}^{\text {os }} 12,71,127,130,141,152-154$ ), il y a là tout de même encore un exemple de rhotacisme ( $\Lambda$ v́pı $\pi$ os, $\mathrm{n}^{\circ} 141$, stèle du III ${ }^{\mathrm{e}}$ s.).

113. De telles listes sont bien attestées, on le sait, à Athènes, à Mégare et dans les cités béotiennes (pour la Béotie, voir l'étude récente de Y. Kalliontzis, "An Unpublished Casualty-List from Plataia and the Memory of War in Boeotia", dans N. Papazarkadas [n. 91], p. 332-372). À Érétrie, il n’y en a pas d'exemple assuré, le catalogue $I G$ XII 9, 241 (dont l'intitulé est malheureusement très amputé) ayant probablement une fonction différente, même s'il enregistre les noms de plusieurs dizaines de soldats classés en hoplitai et en psiloi. L’épigramme fragmentaire IG XII 9, 255 (CEG I, 107), datant du début $\mathrm{du} \mathrm{V}^{\mathrm{e}}$ s., pourrait avoir couronné - à la manière attique - une liste de morts à la guerre.

114. Chr. Dunant (n. 55), p. 23 : «Tableau de répartition numérique selon les types de stèles». 
que la confection des monuments et la gravure des inscriptions elles-mêmes ne sont pas sexuellement différenciées, les commanditaires faisant visiblement abstraction de ce critère. C'est d'ailleurs de manière à peu près simultanée, selon toute apparence, que s'est effectué, pour l'ensemble de la population civique, le passage progressif, vers 350-300, à la nouvelle formulation des épitaphes, ce qui n'est sans doute pas sans intérêt d'un point de vue sociologique ${ }^{115}$.

\section{Deux noms androgynes?}

L'identité sexuelle - dans tous les cas, du moins, où il n'y a pas de lien incontestable entre l'épitaphe et la tombe sur laquelle elle se dressait ${ }^{116}$ - ne peut donc s'établir, en définitive, que sur les noms des défunts : c'est l'onomastique, et elle seule, qui permet de différencier les hommes et les femmes. Cela vaut dans presque tous les cas, puisqu'il existe des anthroponymes qui - moyennant cependant un changement dans l'accentuation, chose essentielle pour les locuteurs - pouvaient servir à désigner des personnes des deux sexes. Contrairement à ce que l'on pourrait croire, ces noms en quelque sorte androgynes ou - pour employer un adjectif plus actuel - «unisexes» ne sont pas de genre neutre, car les appellatifs neutres, qui ont formé beaucoup de noms propres en grec (surtout des diminutifs), ne sont appliqués, on le sait, qu'à des femmes : il n'y en a, du reste, aucun exemple parmi nos documents, tandis que le lot publié par Chr. Dunant (sans parler du matériel onomastique érétrien en général) en offre au moins un exemple ${ }^{117}$. En fait, ce sont essentiellement les anthroponymes en $-i \varsigma$ qui, selon les cas, peuvent faire problème : c'est ainsi qu'Olivier Masson, dans l'une de ses contributions à l'onomastique grecque, pouvait évoquer «les malheurs

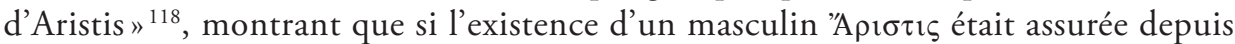

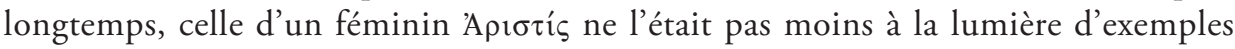
alors récemment publiés. Ici, on a le cas de $\Sigma_{1} \lambda \eta v i \varsigma$ 'A $\pi \mathrm{o} \lambda \lambda \omega v i ́ o v(27)$, qui n’est en

115. On ne connaît pratiquement pas à Érétrie d'épitaphes de femmes où le nom du père soit complété par la mention de celui du mari. Pour l'intérêt sociologique de tels documents, surtout lorsque les démotiques sont indiqués, voir A.-M. VéRILHAC, Cl. VIAL, Le mariage grec, du vt s. av. J-C. à l'époque d'Auguste, BCH Suppl. XXXII (1998), p. 85-87.

116. Les exemples étant très rares partout, on citera celui, remarquable, qu’a fourni récemment la fouille, à Lamia, d'une tombe pour un Érétrien précisément : voir Bull. ép. 2012, 235.

117. Chr. Dunant (n. 55), nº 84 : Lînov. Pour ce type de noms, voir la mise au point de C. Dobias et L. Dubois dans leur introduction aux Onomastica Graeca Selecta d'O. MAsson, I-II (1990), p. XIII-XIV, citant ce nom même comme exemple (mais sans renvoi spécifique). Cf. aussi IG XII 9, 736 (Eînov), 748

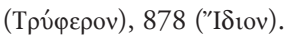

118. «Pape-Benseleriana I : Les malheurs d'Aristis», ZPE 14 (1974), p. 179-183 = Onom. Gr. Sel. I, p. 205-209 + 242 pour des compléments sur ce nom et le nom Elpis. Plus généralement, voir ses « Remarques sur les noms de femmes en grec», MH 47 (1990), p. 129-138 (= Onom. Gr. Sel. III, p. 93-102). 
réalité guère douteux, puisque ce nom - nouveau selon toute apparence en Eubée est manifestement la forme féminine du masculin $\Sigma i \lambda \eta v o ́ \varsigma$, connu à la fois comme appellatif et comme anthroponyme ${ }^{119}$ : de fait, les deux exemples que l'on avait jusqu'ici de $\Sigma_{\imath} \lambda \eta v i ́ s$ dans le monde grec sont clairement féminins ${ }^{120}$. L'hésitation,

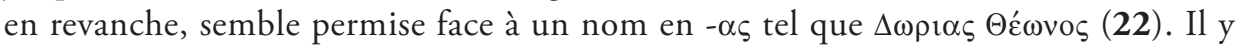
a certes de rares exemples à peu près assurés du masculin $\Delta \omega p i ́ \alpha \varsigma$ (attesté par ailleurs comme hydronyme pour de lointains cours d'eau ${ }^{121}$ ), notamment un, littéraire, à Athènes au $\mathrm{IV}^{\mathrm{e}}$ s. ${ }^{122}$, puis, beaucoup plus tard, dans une remarquable inscription funéraire d'Amastris en Paphlagonie ${ }^{123}$. Mais si les attestations du féminin $\Delta \omega \rho$ ó́ (ethnique correspondant au masculin $\Delta \omega \rho \imath \varepsilon v ́ \varsigma)$ ne sont, tout compte fait, pas infiniment plus nombreuses ${ }^{124}$, il est important de noter qu'à Athènes un exemple épigraphique, bien curieusement rangé du côté des mâles dans le Lexicon of Greek Personal Names, doit être, tout au contraire, tenu pour un nom féminin, puisque cette $\Delta \omega p$ pó́ $\varsigma$ a consacré à Aphrodite l'image d'un sexe de femme ${ }^{125}$ ! En Eubée même, au surplus, la nécropole de Chalcis a livré depuis longtemps une belle stèle à relief de type attique (réapparue il y a peu dans le commerce des antiquités) représentant une

119. Cf. Fr. Bechtel, HPN, p. 568, citant un exemple thasien chez Hippocrate (cf. LGPN I, s.v. no 11 ) et rapprochant ce nom du dérivé beaucoup plus commun $\Sigma \imath \lambda \alpha v i \omega v$. La variante éolo-dorienne $\Sigma \imath \lambda \alpha v o ́ \varsigma$ est également bien connue (y compris à Athènes et en pays ioniens).

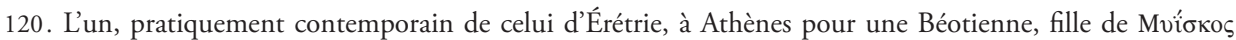
(IG II $^{2} 8221$ : cf. LGPN III.B, s.v. « $\Sigma \imath \lambda \alpha v i \varsigma \varsigma$ ", bien que le nom ait été atticisé en $\Sigma \imath \lambda \eta v i ́ \varsigma$ sur cette stèle à relief de confection attique), l'autre du $\mathrm{II}^{\mathrm{e}}$ s. à Milet dans l'inscription du navarque Antigonos et de toute sa famille sur plusieurs générations (ibid. V.B, s.v., où l’on est renvoyé à P. Herrmann, Chiron 17 [1987], p. 183-190; cf. aussi A. Chaniotis, EA 10 [1987], p. 41-44; SEG XXXVII 992). À noter que P. Herrmann, loc. cit. (p. 184 et n. 35) ne pouvait alléguer que deux exemples de ce nom, l'un et l'autre clairement fictifs, dans Anth. Gr. VII 456, 1, et XI 409, 2.

121. Cf. W. Pape, G. E. Benseler, Wörterbuch der griechischen Eigennamen ${ }^{3}$ (1870), s.v.

122. LGPN II, s.v. $\mathrm{n}^{\circ} 2$ (pour le $\mathrm{n}^{\circ} 1$, voir infra, $\mathrm{n} .124$ ), exemple emprunté à la comédie, et donc peut-être fictif. Dans une épitaphe (hellénistique?) de Tanagra, le doute est certes théoriquement permis (IG VII 498 ; cf. LGPN III.B, s.v.), mais les chances sont en réalité très grandes pour qu'il s'agisse là aussi d'une femme.

123. SEG XXXV 1327 et XL 1163, datée très précisément de l'an 155 apr. J.-C. Je remercie D. Ackermann d'avoir attiré mon attention sur ce document, qui ne semble pas avoir été commenté dans Bull. ép. 1989, sinon par un renvoi global, sous le no 733, à Chr. MAREK, EA 6 (1985), p. 133-156.

124. Ainsi notamment une femme de ce nom dans un inventaire de l'Asklépieion d'Athènes (cf. LGPN II, s.v. $\mathrm{n}^{\circ} 1$ ) et une autre dans une épitaphe hellénistique récemment découverte à Amphipolis, $\Delta \omega p i \grave{\varsigma} \varsigma$ $\mathrm{X} \alpha \mathrm{p}_{\mathrm{o} \delta} \mu \alpha \varsigma$, le second nom étant tenu pour un métronyme, puisque cette stèle à relief montre une scène de dexiôsis entre deux femmes (SEG XLIX 675 : cf. LGPN IV, s.v., où l'exemple est également isolé pour la Macédoine).

125. IG $\mathrm{II}^{2} 4635$ (avec l'accentuation correcte, tandis que l'accentuation masculine est adoptée dans LGPN II, s.v. $\mathrm{n}^{\circ} 1$ ) : "supra titulum sculpta est pars muliebris" (J. Kirchner renvoyant justement au $\mathrm{n}^{\circ} 4575$, autre ex-voto à Aphrodite par une femme, avec la même représentation anatomique). 


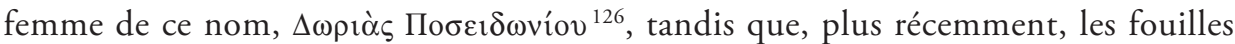

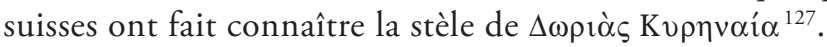

\section{Anthroponymes féminins}

Quelques-uns des noms portés par les femmes de ce modeste secteur de la nécropole occidentale méritent d'être commentés. On peut certes passer assez vite sur les deux $\Theta \varepsilon \tau \tau \alpha \lambda \eta ́$, mère et fille (selon toute probabilité : voir ci-dessus) dans la stèle à relief $\mathbf{1}$. Ce nom tiré d'un ethnique n'est pas rare, du mois au masculin et sous la forme commune $\Theta \varepsilon \sigma \sigma \alpha \lambda{ }^{\prime} \varsigma^{128}$; le recours à cette forme présumée attique ne doit pas être regardé comme résultant nécessairement d'un atticisme à mettre sur le compte du sculpteur et lapicide de la stèle, puisque cette pièce pourrait fort bien, en réalité, être sortie d'un atelier local ${ }^{129}$ : car il faut se souvenir que dans le dialecte ionien d'Érétrie, la graphie avec double tau correspond à une évolution phonétique commune à l'Attique, à la Béotie et à l'Eubée face au double sigma de la koinè ${ }^{130}$. La plupart des autres anthroponymes féminins appartiennent à des catégories connues, mais ils ne sont pas tous banals pour autant. Soulignons en particulier l'extrême rareté du nom 'A $\pi \alpha ́ \lambda \eta$ (2), qui, à n'en pas douter, est la forme féminine de l'adjectif $\alpha \pi \alpha \lambda$ ó $\varsigma$ "tendre, doux", bien attesté dès l'époque homérique ${ }^{131}$. À première vue, on imaginerait qu'un tel nom soit partout répandu, et pour les femmes en particulier : or, il n'en est rien ${ }^{132}$, puisque le masculin 'A $\pi \alpha \lambda \circ \varsigma$ (avec l'accent proparoxyton) ne se trouve guère attesté qu'à Smyrne ${ }^{133}$, tandis que pour 'A $\pi \alpha ́ \lambda \eta$, on n'avait jusqu' ici, chose remarquable, que deux occurrences très tardives et passablement

126. IG XII 9, 1012. Pour les circonstances de la trouvaille dès avant 1850 et le sort postérieur de la stèle parvenue en France, cf. Bull. ép. 2015, 352.

127. Chr. Dunant (n. 55), n 177 (SEG XXVIII 725), où l'éditrice renvoie aussi, pour "ce nom pas très fréquent", aux deux attestations athéniennes. Cf. J. et L. RoBert, Bull. ép. 1979, 349 et maintenant LGPN I, s.v.

128. Un exemple érétrien sous cette forme, mais connu seulement par des inscriptions de Delphes : cf. $L G P N$ $\mathrm{I}$, s.v. $\mathrm{n}^{\circ} 9$.

129. Voir supra, p. 196-198.

130. Sur cette caractéristique régionale transcendant les grands ensembles dialectaux, voir en dernier lieu

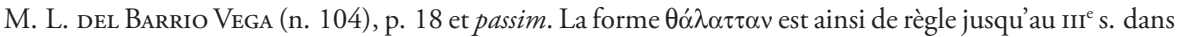
les proxénies d'Érétrie (voir D. KNoepfler [n. 75], index grec, s.v.). Rappelons ici que le héros local Narkissos (dans les sources littéraires) devait en réalité s'appeler Narkittos à Érétrie et à Oropos, comme

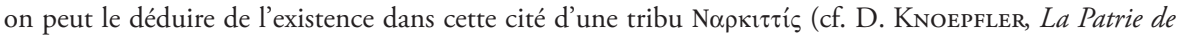
Narcisse [2010], p. 105-106).

131. Cf. P. Chantraine, DELG, s.v. (qui ne cite aucun anthroponyme tiré de cet adjectif).

132. Ce qui explique sans doute son absence dans le répertoire classique de Fr. BECHTEL, là où on l'attendrait (HPN, p. 479-484 : "Der Name knüpft an das körperliche Wesen an»). Il ne semble pas qu’il ait attiré l'attention des érudits jusqu'ici.

133. LGPNV.A, s.v. $\mathrm{n}^{\circ} 1$ (époque impériale). 
périphériques, en Campanie d'une part et en Cilicie de l'autre ${ }^{134}$. L'apparition de ce nom dès le $\mathrm{IV}^{\mathrm{e}} \mathrm{s}$. dans l'ionienne Érétrie, au cœur de la Vieille Grèce, est donc un apport intéressant.

Parmi les femmes dont les noms sont encore dépourvus de patronyme (et qui pourraient, dès lors, appartenir aux premières générations d'occupants de la nécropole), quelques-uns - quoique moins rares - attirent cependant l'attention : ainsi, très particulièrement, 'E $\mu \pi \varepsilon \delta o v i ́ \kappa \eta(6)$. Cette femme pourrait éventuellement ne faire qu'un avec l'Érétrienne de ce nom figurant dans une inscription de la même époque, où le nom apparaît au génitif en tant que métronyme d'une autre femme, $\Phi_{1} \lambda_{0} \xi_{\xi}^{c} v \eta^{135}$; or, c'est en Béotie que les noms formés sur ce radical paraissent avoir eu un caractère véritablement épichorique $^{136}$. Il se pourrait donc que notre 'Е $\mu \pi \varepsilon \delta$ ovíkn ait été, elle aussi, d'origine béotienne (quoique les composés en -víkn soient par ailleurs communs en Eubée dès l'époque classique). Le nom Eủpór $\alpha$ (7) - dont la lecture est assurée, en dépit de la disparition de la lettre initiale - n'a pas, lui, un caractère épichorique : emprunté à la sphère mythologique ou plus généralement religieuse (puisqu'une Déméter Eurôpè était honorée à Lébadée ${ }^{137}$ ), ce nom pourrait, théoriquement, avoir essaimé en maintes régions dès l'époque archaïque. Pourtant, force est de constater que l'aire de diffusion en a été assez limitée dans l'espace comme dans le temps, les premières attestations du nom n'étant pas antérieures à la fin $\mathrm{du} \mathrm{IV}^{\mathrm{e}} \mathrm{s}$. (de fait, la stèle érétrienne date au plus tôt des alentours de $300^{138}$ ) et restant ensuite confinées à la Grèce propre (aucun exemple, chose significative, n'est recensé pour la Grèce d'Occident ni non plus pour l'Asie Mineure la plus hellénisée $\left.{ }^{139}\right)$. On peut dès lors se demander si le relatif succès de ce nom en «Europe» proprement dite ne serait pas à mettre en relation, pour une part au moins, avec la montée en puissance de la famille royale macédonienne, où, en 336, la dernière épouse de Philippe II, Kléopatra (dont le nom était promis au succès que l'on sait) donna naissance à une fille dénommée Eurôpè ${ }^{140}$, qui certes, disparut trop tôt pour connaître la gloire réservée à d'autres princesses.

134. LGPNV.B, s.v. $\mathrm{n}^{\circ} 1$; pour la Campanie, cf. ibid. III.A, s.v. $\mathrm{n}^{\circ} 1$.

135. LGPN I, s.v. $\mathrm{n}^{\circ} 1$ (nom tout à fait isolé dans ce volume) avec renvoi erroné à $A E p h$ 1984, p. $135 \mathrm{n}^{\circ} 46$. En réalité, ce cippe trouvé en 1981 (Musée d'Érétrie, inv. n 13561) paraît être resté inédit.

136. Voir LGPNIII.B, p. 133. Ce nom pourrait donc être ajouté à la liste des anthroponymes étudiés naguère par F. Marchand, "Rencontres onomastiques au carrefour de la Béotie et de l'Eubée», dans N. BADOUD (éd.), Philologos Dionysios. Mélanges offerts au professeur Denis Knoepfler (2011), p. 343-376.

137. Pausanias, IX 39, 2-4; cf. A. Schachter, Cults of Boiotia I (1981), p. 156-157.

138. Cette attestation doit être plus ancienne, en tout cas, que l'exemple delphique retenu par Fr. BechteL, $H P N$, p. 579 ( Personennamen aus Namen von Heroinen»).

139. Comme on le voit maintenant grâce aux deux tout récents volumes du LGPNV.A (2010) et V.B (2012).

140. Cf. LGPNIV, s.v. n 1 , avec renvoi à A. B. TATAKI, Macedonians Abroad (1998), p. $316 \mathrm{n}^{\circ} 59$. Pour cette question de l'influence des noms dynastiques féminins sur l'onomastique commune, voir maintenant 


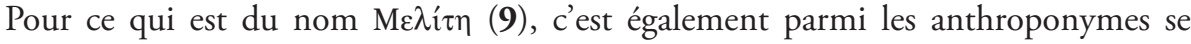
rattachant au monde des dieux et plus spécialement au «Kreis der Meeres- und Flussgötter» que le rangeait Fr. Bechtel ${ }^{141}$, à cause de l'existence, parmi les Néréides, d'une nymphe ainsi nommée, en alléguant d'ailleurs comme exemple une première

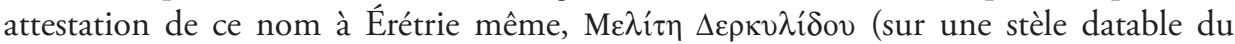
$\mathrm{III}^{\mathrm{e}}$ s. av. J.-C. ${ }^{142}$ ). Il n'y aurait donc, aux yeux de ce savant, aucun lien entre ce nom héroïque et les nombreux anthroponymes formés sur l'appellatif $\mu \varepsilon ́ \lambda \iota \sigma \sigma \alpha$, «abeille», bien que ceux-ci, dans l'Érétriade, se rencontrent sous la forme dialectale attendue Mé̀ $\imath \tau \tau \alpha$

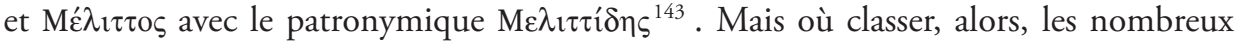

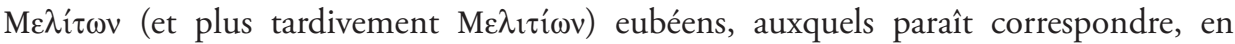

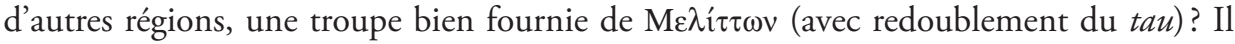
faut, à tout le moins, supposer des interférences entre ces deux familles que l'on pourrait qualifier de "paronymiques».

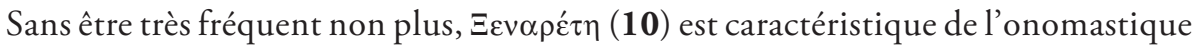
eubéenne, avec ce goût marqué - aristocratique, peut-on penser - pour les composés

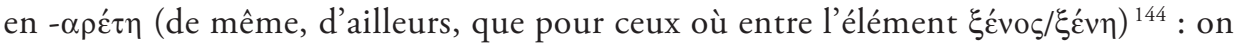
connaissait déjà, à Érétrie même, plusieurs $\Delta \eta \mu \alpha \rho \varepsilon^{\prime} \eta \eta$ de la haute époque hellénistique, dont une a fait son apparition tout récemment sur une stèle remployée à proximité

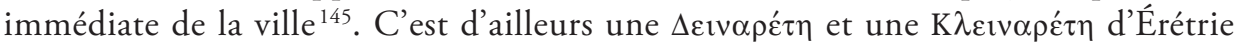
qui servent d'exemples pour ces noms dans le répertoire de Bechtel ${ }^{146}$. À la même

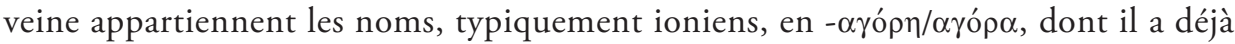

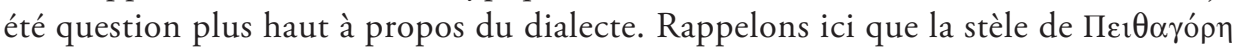
(12) n'est pas nécessairement plus ancienne - et pourrait même être plus récente -

Y. Brioux, W. Clarysse, «Would You Name Your Child after a Celebrity? Arsinoe, Berenike, Kleopatra, Laodike and Stratonike in the Greco-Roman East», ZPE 200 (2017), p. 347-362.

141. $H P N$, p. 469 ; cf. p. 584 pour les noms tirés de celui de l'abeille.

142. IG XII 9, 675. Pour la date, que nous avons établie sur la base d'un examen de la pierre, cf. LGPNI, s.v. $\mathrm{n}^{\circ} 3$; le nom est également attesté à Chalcis (ibid., n 2) et ailleurs. La forme Me $\lambda \dot{i} \tau \alpha$ (à moins qu'il ne

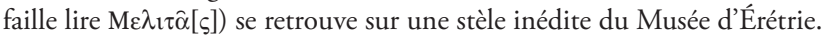

143. LGPN I, s.vv.

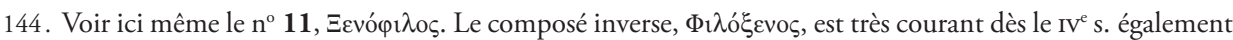
(cf. D. KNoepfler, "Le tronc à offrandes d'un néocore érétrien", $A K 41$ [1999], p. 101-116, à propos d'un nouvel exemple; un autre encore, fourni par une stèle inédite, pourrait remonter aux alentours de 400).

145. Ce cippe, datable du Iv $s$. (grandes lettres gravées sur un bandeau réservé), provient de la fouille du site paléochrétien de H. Paraskevi : cf. D. KNoepfler, Bull. ép. 2015, 346 en p. 504 (rétablissement du nom lu incorrectement par l'éditrice). Pour les exemples antérieurement connus à Érétrie, cf. LGPN I, s.v. $\mathrm{n}^{\text {os }} 2-4$.

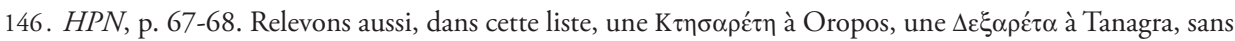
parler des masculins formés sur le même élément (cf. LGPN III.B, s.vv. avec les références actualisées). 
que celle de $\mathrm{K} \lambda \varepsilon \alpha \gamma o ́ p \alpha(8)$, qui a pourtant déjà la désinence commune empruntée à l'attique ou, plus largement, à la koinè ${ }^{147}$ : l'explication de ce conservatisme - si la date de l'épitaphe $\mathbf{1 2}$ devait être abaissée au début du III $^{\mathrm{e}}$ s., comme pourrait le suggérer l'écriture - réside peut-être dans le fait que le nom Пعi $\theta \alpha \gamma o ́ p \eta$, qui semble effectivement bien plus rare (c'est un hapax, pour le moment, en Eubée), était demeuré l'apanage d'une seule famille aristocratique. Le nom $\Delta \eta \mu \eta \tau \rho i ́ \alpha$ (4), lui, est certes infiniment plus banal: il faut relever cependant que l'on a là un exemple parmi les plus anciens, pour l'Eubée et la Béotie en tout cas ${ }^{148}$, de ce théophore appelé à se répandre partout (comme son équivalent masculin) à partir de la basse époque hellénistique.

Parmi les noms de femmes qui sont accompagnés d'un patronyme - dont la présence est un indice, on l'a vu, mais nullement une preuve, que les épitaphes doivent appartenir au plus tôt à la seconde moitié du $\mathrm{IV}^{\mathrm{e}} \mathrm{s}$. - il faut signaler en priorité le nom 'A $\rho \chi \varepsilon \mu o ́ v \eta$ (20), nom composé de deux éléments bien connus, mais qui semble nouveau aussi bien sous cette forme féminine que sous la forme masculine A $\rho \chi \varepsilon ́ \mu o v o \varsigma$ : quel contraste

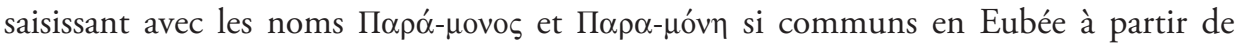
l'extrême fin du IV ${ }^{e}$ s. ${ }^{149}$ (mais encore complètement absents, on peut le noter, parmi les stèles publiées ici, alors qu'il s'en trouve déjà près d'une dizaine d'exemples dans le lot de $1978^{150}$, pourtant contemporain à très peu de chose près). Très caractéristique également de l'Eubée centrale est le nom Bió $\eta^{151}$ (21), forme féminine du masculin

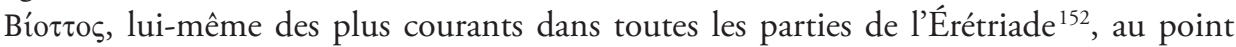
qu'on peut le tenir pour fondamentalement épichorique dans ce pays : c'est ce que nous

147. Une évolution très claire se marque dans l'Ionie même pour ces noms, qui ont la forme - $\alpha \gamma o ́ p \eta \varsigma$ dans une célèbre liste de souscription de Colophon à l'extrême fin du Iv s., alors que les formes en - $\alpha \gamma o ́ p \alpha \varsigma$ se sont généralisées dès le milieu du siècle suivant : voir D. RousseT, "La stèle des Géléontes au sanctuaire de Claros", JS 2015, p. 3-98, en p. 95.

148. Cf. LGPN III.B, s.v.

149. Voir A. Morpurgo-Davies, «Après Michel Lejeune : l'anthroponymie et l'histoire de la langue grecque», CRAI 2001, p. 157-173, en particulier p. 167-172. Cf. D. KNOEPfler (n. 110), p. 57 et n. 22 (renvoi à

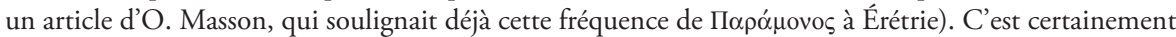
de l'Eubée centrale, au vu de la chronologie des attestations, que ces noms se sont répandus dans la Béotie voisine.

150. Parmi eux, il s'en trouve déjà quatre - tous datables du $\mathrm{III}^{\mathrm{e}}$ s. - sans patronyme (Chr. DunANT [n. 55], $\mathrm{n}^{\text {os }} 73-76$ ), tandis que deux autres, de la même époque, ont un patronyme (ibid., $\mathrm{n}^{\text {os }} 149-150$; le patronyme П $\alpha \rho \alpha \mu$ óvov figure en outre sur les $\mathrm{n}^{\text {os }} 150$ et 152$)$. Pour un exemple ancien, cf. supra, n. 104.

151. Cf. $L G P N$ I, s.v. $\mathrm{n}^{\text {os }}$ 1-6: une demi-douzaine d'exemples érétriens à partir du III $^{\mathrm{e}}$ s., auxquels s'ajoute la mention d'une prisonnière chalcidienne dans un acte d'affranchissement de Delphes, sous la forme Bió $\alpha$ (SGDI 1844, 1. 4; D. Mulliez, CID V nº 97, à paraître; cf. A. Bielman, Retour à la liberté. Libération et sauvetage des prisonniers en Grèce ancienne [1994], p. 270 n. 239 et 292 avec la n. 134).

152. Comme cela ressort de la liste des quelque 30 occurrences érétriennes dans le LGPN I, s.v. $\mathrm{n}^{\text {os }}$ 6-39. 


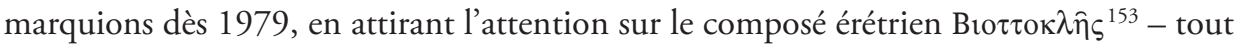
à fait sui generis - alors récemment apparu et demeuré depuis lors, selon toute apparence, un hapax absolu. Pour ce qui est du dérivé Níкv $\lambda \lambda \alpha$ (26), il est loin d'avoir été aussi populaire, mais un autre exemple érétrien de ce nom pourrait se dissimuler dans une épitaphe amputée à droite, où la restitution Níkv $[\lambda \lambda \alpha]$ s'avère effectivement, aujourd'hui, tout aussi attractive que $\mathrm{N} \imath$ vó$[\lambda o \varsigma]$, compte tenu de la lacune ${ }^{154}$; dans le reste de la Grèce propre, les attestations en sont éparses et peu nombreuses : deux à Athènes, une seule dans tout le Péloponnèse, une autre en Thessalie (mais, chose notable, aucune en Béotie), auxquelles sont venus s'ajouter trois exemples macédoniens ${ }^{155}$. On relèvera que c'est la nécropole d'Érétrie, avec sa riche collection d'épitaphes d'étrangers, qui a fait connaître

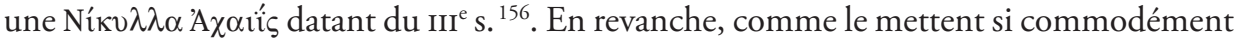
en évidence les deux premiers volumes, récemment parus, du $L G P N \mathrm{~V}$, ce dérivé n’a

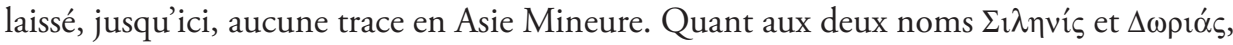
ils ont déjà fait ci-dessus l'objet d'un commentaire suffisant ${ }^{157}$.

Achevons donc cette notice sur l'onomastique féminine du nouvel ensemble par la

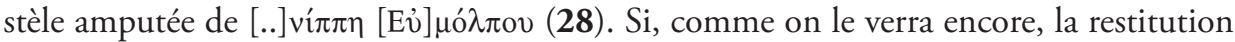
du patronyme est certaine, celle de l'idionyme ne peut être tentée qu'exempli gratia, car en dépit du nombre très réduit des lettres manquantes (certainement pas plus de

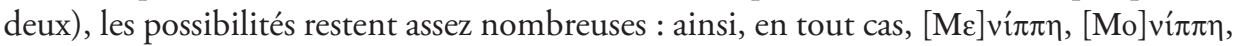

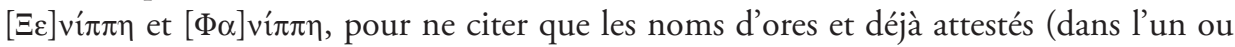
l'autre genre). On sait avec quelle constance et avec quelle ingéniosité les Érétriens se sont appliqués à rechercher - et souvent à créer de toutes pièces pour leur usage exclusif - des composés intégrant l'appellatif hippos comme premier ou second élément, au point que ce trait de leur onomastique - y compris sur le mode féminin - transparaît dans presque tous les documents de leur cité ${ }^{158}$. Aussi est-on presque étonné de n’avoir à enregistrer ici qu'un seul nom relevant de cette famille anthroponymique, alors que, dans son recueil, Chr. Dunant pouvait recenser pas moins de dix cas, sur quelque 160 épitaphes concernant

153. “Contributions à l'épigraphie de Chalcis, II», BCH 101 (1979), p. 183 avec la n. 93, à propos d'un

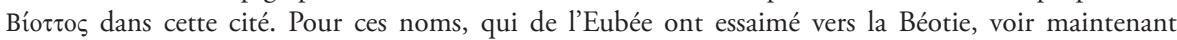
F. Marchand (n. 136), p. 357-369.

154. IG XII 9, 444. Cf. LGPN I, s.v. n 1, où nous envisagions déjà ces deux possibilités.

155. D'après $L G P N$ II, III.A, III.B et IV, s.vv.

156. IG XII 9, 822. Cf. LGPN III.A, s.v. $\mathrm{n}^{\circ} 1$ (avec l'adoption de notre datation).

157. Voir ci-dessus, p. 209 : «Deux noms androgynes?».

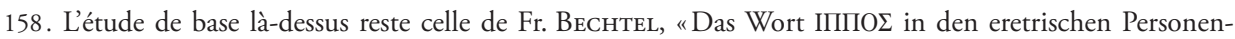
namen", Hermes 35 (1900), p. 325-331 (=Kleine onomastische Studien [1981], p. 98-103). Pour un nouvel exemple d'un tel nom dans le dème de Lakè, cf. D. Knoepfler, G. Ackermann, «Phulè Admètis : un nouveau document sur les institutions et les cultes de l'Érétriade", CRAI 2012 (2013), p. 905-949; sur la faveur dont jouissait l'hippotrophia dans ce dème en particulier, cf. D. KNOEPfLeR (n. 110), p. 73. 
des Érétriens, se répartissant d'ailleurs très inégalement entre les hommes (2 occurrences) et les femmes (8 occurrences) ${ }^{159}$. Cet écart, si notable soit-il, parait fortuit. Assurément, il a pu exister des différences dans la fréquence de ces anthroponymes en fonction de la position des dèmes à l'intérieur même du territoire ${ }^{160}$, certains ayant une configuration et des ressources mieux adaptées que d'autres à l'élevage intensif du cheval. Mais si l'on considère la société érétrienne dans sa globalité, on constatera qu'il y a une plus grande diversité de noms masculins en hippos que de noms féminins de cette famille: car si l'homme tirait gloire des multiples facettes de son savoir-faire en matière d'élevage, de dressage et plus généralement de conduite de ces animaux, la femme pouvait, à tout le moins, s'enorgueillir de donner naissance - telle une jument sélectionnée pour la reproduction! - aux plus beaux spécimens de la race ${ }^{161}$.

\section{Anthroponymes masculins}

Pour ce qui est des anthroponymes masculins, considérons d'abord ceux qui apparaissent seuls, dans les épitaphes appartenant donc, a priori (mais sans certitude), à la catégorie des monuments les plus anciens. De fait, il s'en trouve plusieurs, parmi eux, qui ont une

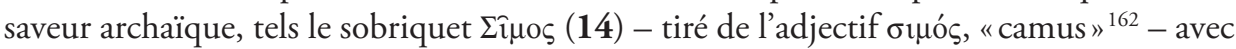
le dérivé $\Sigma \mu_{\mu i ́ \alpha \varsigma}$ (13), déjà connu en Eubée par plusieurs exemples de la haute époque hellénistique ${ }^{163}$, ou l'hypocoristique $\Phi$ ôvos $(\mathbf{1 5})$ - très bien attesté à Délos et à Érétrie même ${ }^{164}$ (à côté des formes ioniennes $\Phi \alpha ́ v \eta \varsigma / \Phi \alpha v \eta \varsigma^{165}$ ) - ou encore le dérivé X $\alpha$ pîvos (17), appartenant à une famille de noms bien représentée à Érétrie, mais connue également en

159. Chr. Dunant (n. 55), p. 27. Il s'agit des $n^{\text {os }} 13,42,60,66,94,119,132$ (même nom féminin que dans le $\left.n^{\circ} 13\right), 141,145$ et 153 .

160. Sur cet aspect des choses, avec la critique de la théorie de W. WALlace, "The Demes of Eretria", Hesperia 16 (1947), p. 115-146, sur la localisation des dèmes érétriens en fonction de ce seul critère, voir D. Knoepfler (n. 110), p. 55.

161. Sur l'élevage des chevaux à Érétrie, voir l'attachante étude de P. Simon et S. Verdan, AK 57 (2014), p. 1-21, avec nos observations dans Bull. ép. 2015, 347. Partant de la remarquable association iconographique entre la représentation d'un coït humain et celle de la saillie d'une jument par un étalon sur un vase érétrien d'époque géométrique, les auteurs montrent précisément que, dans l'esprit du peintre, les deux activités tendaient au même but, soit la bonification de l'espèce.

162. Pour cette famille de noms, cf. Fr. Bechtel, HPN, p. 490-491 ( Stumpfnasig»), avec un exemple

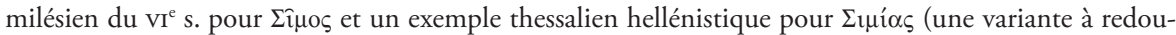
blement expressif, $\Sigma \mu \mu i_{\alpha}$, est connue notamment à Tanagra), tandis qu'Érétrie a fourni à Bechtel

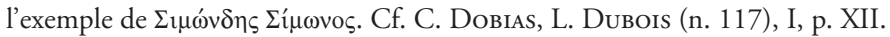

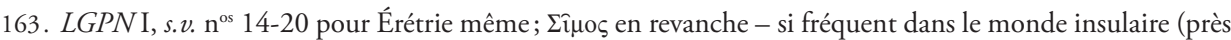
de 100 exemples connus en 1987) - n'était jusqu'ici apparu qu'une seule fois à Érétrie et seulement au $\mathrm{I}^{\mathrm{er}}$ s. (ibid., s.v. $\left.\mathrm{n}^{\mathrm{o}} 30\right)$.

164. LGPN I, s.v. $\mathrm{n}^{\text {os }}$ 2-26 (Délos) et 29-33 (Eubée).

165. Sur ces deux formes, voir O. Masson, RN 26 (1984), p. 55 (=Onom. Gr. Sel. I-II, p. 434), qui ne mentionne pas la forme $\Phi \hat{\alpha} v o \varsigma$, ni là ni ailleurs, semble-t-il. 
plusieurs autres régions ${ }^{166}$. C’est le cas aussi de $[\mathrm{M \varepsilon}] \lambda \lambda_{\alpha}{ }^{\prime} \theta$ ios $(\mathbf{1 8})$, la restitution étant là pratiquement assurée pour deux raisons convergentes : la première est que les composés

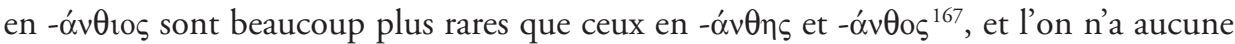
occurrence ancienne, par exemple, du nom $\Phi_{1} \lambda \alpha^{\alpha} v \theta \operatorname{los}^{168}$ (théoriquement possible ici); de

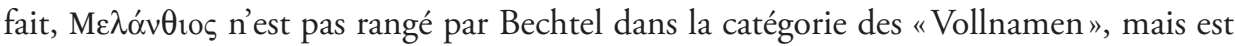
tenu, avec raison, pour un nom emprunté directement au monde des héros homériques

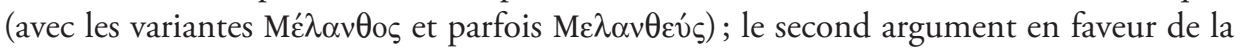
restitution adoptée, c'est l'existence de plusieurs Eubéens de ce nom à date ancienne,

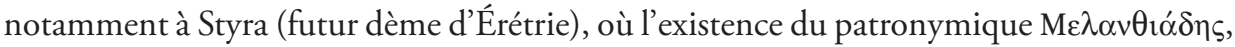
beaucoup plus rare, est dûment signalée par Bechtel ${ }^{169}$. Par ailleurs, cependant, il s'agit d'un nom panhellénique, susceptible d'apparaître en toute contrée.

On peut en dire autant, sans doute, de $\Delta$ óкıнь (5), tiré sans changement de l'adjectif

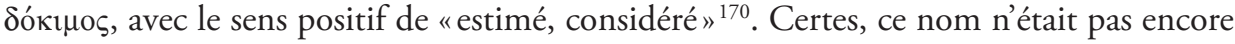
attesté en Eubée même; dans le reste de la Grèce continentale, il est assez peu répandu et pas avant le $\mathrm{III}^{\mathrm{e}}$ s., sauf à Athènes ${ }^{171}$. Mais il paraît avoir été particulièrement populaire, dès le $\mathrm{IV}^{\mathrm{e}}$ s., en Épire, d'où il a pu essaimer vers l'Italie et la Sicile ${ }^{172}$. En Macédoine, deux porteurs de ce nom - qui n'en font sans doute qu'un - sont attestés dans l'entourage d'Alexandre le Grand et d'Antigone le Borgne ${ }^{173}$. En Asie Mineure, les exemples en sont nombreux, en Bithynie et surtout dans l'Ionie du Nord, Smyrne et Éphèse, comme aussi à Milet et à Héraclée de la Salbakè, mais il faut relever qu'ils sont pratiquement tous de

166. L'exemple du nom chez Fr. Bechtel, HPN, p. 466-468, est ainsi celui d'un Halicarnassien de date indéterminée. Pour ce qui est de l'Eubée, Xapîvos y était déjà connu mais seulement dans le Sud de l'île (LGPN I, s.v. $\mathrm{n}^{\text {os }} 10-12$ ), de même que Xopías paraît avoir été plus particulièrement populaire dans la région de Styra (cf. D. KNoepfler [n. 75], p. 244-245).

167. Fr. Bechtel, $H P N$, p. 55-56, qui ne trouvait guère à alléguer que Aptóv $\theta 105$, nom du navarque béotien dans l'armée de Lysandre.

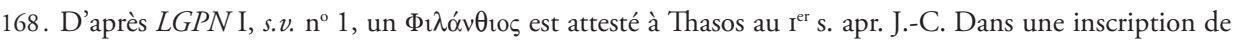
Milet à la fin du $\mathrm{III}^{\mathrm{e}}$ s. cette forme n'est apparemment qu'une graphie fautive, à côté du plus commun

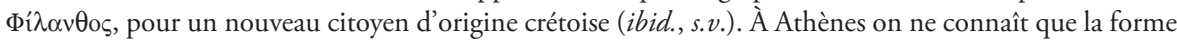

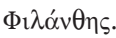

169. HPN, p. 574, avec renvoi aux tablettes $I G$ XII 9, 52, 1. 270-271. On attend une nouvelle édition de cette série de documents : cf. Fr. Dell’Oro, dans A. Inglese (éd.), Epigrammata 3 (2015), p. 17-23.

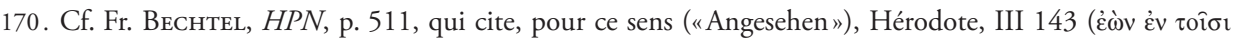

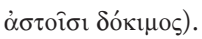

171. LGPN II, s.v. $\mathrm{n}^{\text {os }} 1$ 13. Pour les îles, cf. LGPN I, s.v. $\mathrm{n}^{\text {os }} 1-5$; pour la Béotie-Thessalie, ibid. III.B, s.v. $\mathrm{n}^{\text {os }} 1-2$; pour l'Arcadie, ibid. III.A, s.v. $\mathrm{n}^{\circ} 1$.

172. LGPN III.A, s.v. $\mathrm{n}^{\text {os }} 2-22$.

173. Voir RE, s.v. $\mathrm{n}^{\text {os }} 4-5$, avec la mise au point de A. B. TATAKI (n. 140), p. $304 \mathrm{n}^{\circ} 84$; cf. LGPN IV, s.v. $\mathrm{n}^{\text {os }} 1-2$. 
l'époque impériale ${ }^{174}$. Un certain Dokimos - à coup sûr un des officiers macédoniens mentionnés il y a un instant - était le fondateur de Dokimeion en Phrygie ${ }^{175}$. C'est l'occasion de rappeler que les habitants d'une ville toute voisine de Dokimeion,

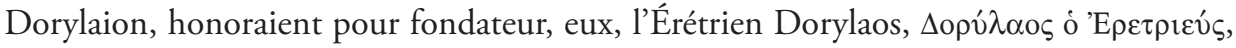
dont l'activité n'est malheureusement pas située précisément dans le temps ${ }^{176}$.

Les "Vollnamen ", ou noms composés, ne sont pas moins anciens, ni moins répandus. Dans les stèles de la première série (sans indication du patronyme), on en trouve déjà

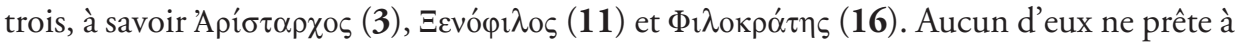
de longs commentaires. Bornons-nous à rappeler qu'en raison de l'ancienneté manifeste de la stèle qui la fait connaitre, cette nouvelle occurrence du composé Apí $\tau \tau-\alpha \rho \chi 0 \varsigma$ - formé de deux éléments bien révélateurs de l'idéal aristocratique qui animait l'élite érétrienne à l'époque de l'Empire athénien encore - est en même temps la plus ancienne

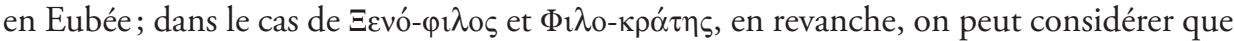
tous les porteurs de ces deux noms dans les inscriptions d'Érétrie appartiennent, grosso modo, à la même génération, celle qui a vécu entre le milieu du IV et le milieu du $\operatorname{III}^{\mathrm{e}}{ }^{\mathrm{e}} \cdot{ }^{177}$.

Dans les stèles de la seconde série (avec patronyme), les noms masculins ne sont plus seulement ceux des défunts eux-mêmes, mais aussi ceux que portent tous les pères des hommes et des femmes inhumés dans cette partie de la nécropole. La plupart se caractérisent par leur plus ou moins grande banalité, à la fois pour le monde grec en

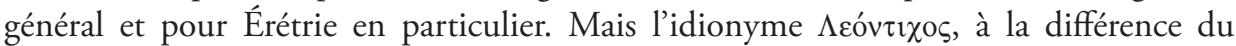
patronyme $\Sigma$ ú $\mu \alpha \chi 0 \varsigma$ (24), est nouveau pour l'Eubée ${ }^{178}$; on ne peut en dire autant ni de l'idionyme $\Lambda v \sigma i ́ \mu \alpha \chi o s$ (sans le rhotacisme attendu ou du moins encore possible à

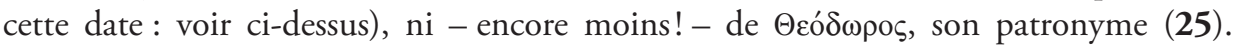

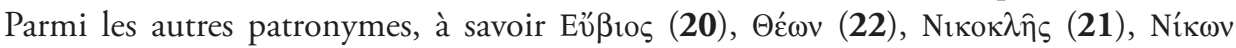

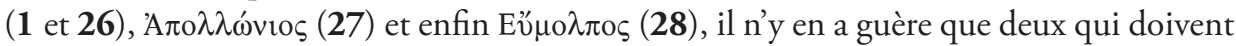

174. Cf. $L G P N$ V.A, s.v. $\mathrm{n}^{\text {os }}$ 1-10 (Bithynie-Ionie), et V.B, s.v. $\mathrm{n}^{\text {os }} 1-15$ (Milet, Carie). Pour les deux exemples de Tabai, la liste éditée par J. et L. RoBert, La Carie II (1954), p. 381 n 194, est maintenant reprise par J.-L. Ferrary, Les Mémoriaux des Délégations de Claros (2015), nº 161.

175. Voir L. Robert, JS 1962, p. 24-25 (= Op. Min. Sel. VII, p. 90-91), avec la note 10 : "Je considère comme assuré que ce Dokimos était le général d'Antigone connu dans l'Histoire et précisément dans la région»; cf. Bull. ép. 1963, 260; À travers l'Asie Mineure (1980), p. 240-244: «Mais Dokimos est un nom qui est le plus souvent Macédonien» (p. 241, avec la n. 11).

176. Cf. $L G P N \mathrm{I}$, s.v. $\mathrm{n}^{\circ} 1$, avec renvoi à deux éditions anciennes d'une inscription d'époque impériale trouvée à Dorylaion même.

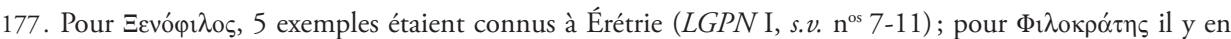
avait 10 (ibid., s.v. $\mathrm{n}^{\text {os }} 33-42$ ). Il va sans dire que ces deux noms sont également très bien attestés ailleurs.

178. Fr. Bechtel, $H P N$, p. 277, considère ce nom comme directement dérivé de l'appellatif $\lambda \dot{\varepsilon} \omega v, \lambda \varepsilon ́ o v \tau o \varsigma$. Dans le monde insulaire, on ne le connaissait encore qu’à Délos et Thasos, avec un exemple aussi en Cyrénaïque ( $L G P N \mathrm{I}$, s.v. $\mathrm{n}^{\text {os }} 1-3$ ). Si les exemples en sont très nombreux à Athènes dès le IV $v^{e}$ s. ( $L G P N \mathrm{II}$, s.v. $\left.\mathrm{n}^{\text {os }} 1-16\right)$, il paraît n’y en avoir aucun, jusqu'ici, dans la Béotie voisine. 


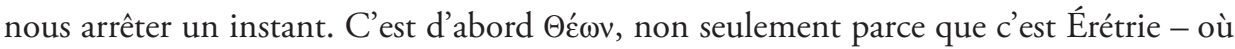
l'on connaît aussi, depuis assez peu de temps, le plus rare féminin @é́ - qui a fourni au répertoire de Bechtel l'exemple de ce nom (de même que celui du patronymique $\Theta \varepsilon \omega ́ v \delta \alpha \varsigma){ }^{179}$, mais aussi en raison d'une possible rencontre prosopographique. En effet, une dédicace à (Artémis) Ilithyie trouvée naguère sur l'Acropole d'Érétrie ${ }^{180}$, a été faite par un personnage dont le nom lui-même est perdu, mais qui était très certainement une femme (étant donné la divinité invoquée) et se trouve être fille d'un dénommé Oécv. Or, cette inscription paraît dater $\mathrm{du}_{\mathrm{IV}}^{\mathrm{e}} \mathrm{s}$. ou en tout cas de la haute époque hellénistique.

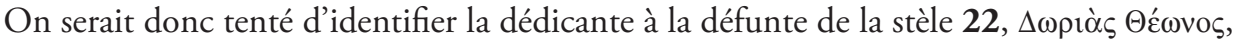
si toutefois le nom Dôrias ne semblait pas un peu court, compte tenu des quelque huit lettres qui sont à restituer devant le patronyme, au vu de la manière dont devait se

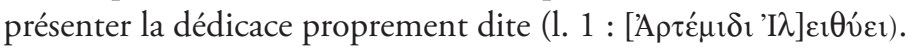

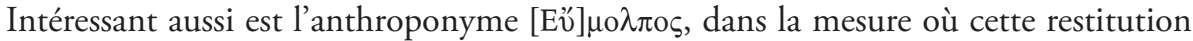
peut être tenue pour assurée; car, si le nom Mó $\lambda \pi$ os, avec ses composés Mo $\lambda \pi \alpha \gamma o ́ \rho \alpha \varsigma$, Mo $\lambda \pi \hat{\theta} \theta \varepsilon \mu ı \varsigma$ et autres, sont bien connus en Ionie, principalement à Milet et dans les colonies milésiennes ${ }^{181}$, il n'y a, en revanche, qu'un nombre extrêmement restreint de composés en $-\mu \mathrm{o} \lambda \pi \mathrm{o}$ : à côté du nom retenu ici, on ne connaît guère, semble-t-il,

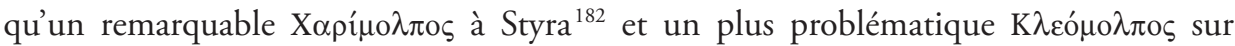
une amphore à figures rouges de Spina ${ }^{183}$, tous deux exclus ici en raison de la place disponible. De fait, l'anthroponyme Eű $\mu \mathrm{\partial} \pi \mathrm{o}$ doit être considéré non pas, à proprement parler, comme un "Vollname», mais comme un emprunt direct à la sphère divine et héroïque ${ }^{184}$. Cela explique qu'à Athènes porter le nom de ce fils de Poséidon - qui était lui-même une divinité - fut impensable avant l'époque impériale ${ }^{185}$; cet interdit s'étendait

179. HPN, p. 207 : le choix de Bechtel a certainement été déterminé par le fait que ces deux Érétriens étaient

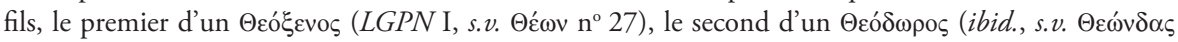
$\mathrm{n}^{\circ}$ 2). Pour le féminin $\Theta \varepsilon \omega ́$, qui reste isolé dans le monde insulaire (mais se trouve en Attique et à Oropos), cf. ibid., s.v. $\mathrm{n}^{\circ} 1$.

180. D. Knoepfler (n. 109), p. 115-127, en particulier p. 125 avec la fig. 5 et la pl. 23, 1-2 (SEG XL 762).

181. Pour ces noms milésiens à Apollonia du Pont et en Thrace, voir A. Avram, dans R. W. V. Catling, F. Marchand (éds), Onomatologos. Studies in Greek Personal Names Presented to Elaine Matthews (2010), p. 375-377.

182. IG XII 9, 56, 1. 427 (allégué par Fr. Bechtel, HPN, p. 323 et 467) : cf. LGPN I, s.v. no 1.

183. SEG IV $160 \mathrm{I} e$, avec la correction suggérée pour la lecture $\mathrm{K} \Lambda \mathrm{EOMO} \Lambda \mathrm{OO}$. De toute façon, le contexte montre qu'il s'agit du nom d'une figure mythique.

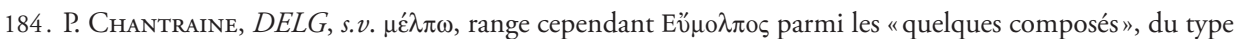
Mo $\lambda \pi \alpha \gamma o ́ \rho \alpha \varsigma$, ce que faisait aussi Fr. BECHTEL, $H P N$, p. 323 (cf. aussi p. 173), en citant pour Eű $\mu \mathrm{o} \lambda \pi \mathrm{s}$ un exemple d'Haliarte, IG VII 1795 (fin du III ${ }^{\mathrm{e}}$ s.), qui est demeuré isolé en Béotie (cf. LGPN III.B, s.v. $\left.\mathrm{n}^{\circ} 1\right)$.

185. Les exemples athéniens sont en effet tous tardifs (cf. LGPNII, s.v. $\mathrm{n}^{\text {os }} 1-11$ ); de même à Milet (LGPN V.B, s.v. $\left.\mathrm{n}^{\text {os }} 2-6\right)$ et ailleurs sur les côtes de l'Asie Mineure. 
d'ailleurs au patronymique Eumolpidès, manifestement réservé à l'usage exclusif du génos

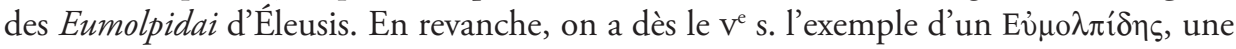
fois encore à Styra, puis d'un Eủ impliqués dans la conjoncture de $379^{186}$. C'est donc du côté de ces deux patronymiques

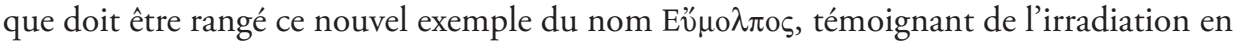

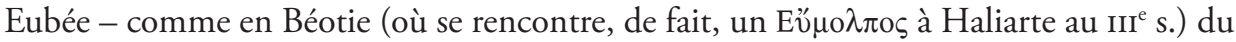
culte éleusinien. Ailleurs, il ne s'est que très faiblement répandu, et à partir de l'époque hellénistique seulement (ainsi en Crète et à Samos); en Eubée même, il n'était connu directement, jusqu'ici, que par un exemple chalcidien du début de la période romaine ${ }^{187}$.

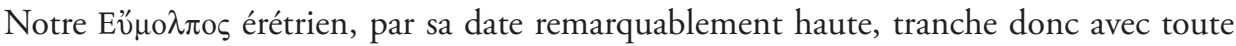
la documentation actuellement connue sur ce théonyme devenu assez tardivement anthroponyme.

\section{L'étranger}

Il n'y a qu'un étranger assuré ${ }^{188}$ parmi les 29 défunts de la nécropole du torrent (environ $4 \%$ ). C'est un chiffre remarquablement faible, car à Érétrie - ville assez cosmopolite aux $\mathrm{IV}^{\mathrm{e}}$ et $\mathrm{III}^{\mathrm{e}} \mathrm{s}$. - la proportion des étrangers parmi les indigènes, tous statuts confondus, est globalement supérieure à $10 \%{ }^{189}$, atteignant même $13 \%$ dans le lot publié en 1978 , avec 25 étrangers sur un total de 186 individus ${ }^{190}$. Il est peut-être permis d'y voir un indice supplémentaire en faveur de l'hypothèse que cette portion de la nécropole aurait

186. $L G P N$ III.B, s.v. nº 1, avec la référence à Plutarque. Mais c'est l'exemple de Styra (IG XII 9, 56, 1. 123), plus ancien, qu’alléguait Fr. BECHTEL, HPN, p. 534 (dans une section réservée aux noms patronymiques

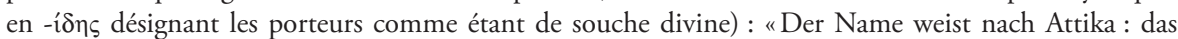

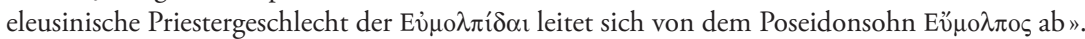

187. IG XII 9, 916, 1. 11, intégré à une nomenclature latine : cf. LGPN I, s.v. $\mathrm{n}^{\text {os }} 4$.

188. Un second se dissimule peut-être dans la stèle fragmentaire 23, puisqu'à la ligne 2 les lettres MA, gravées

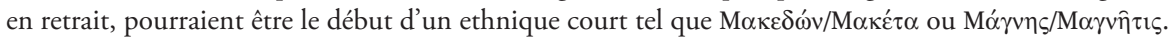

189. Dans la publication de V. Pétrakos (n. 68 [1968 et 1974]), sur un total d'environ 120 stèles, il y a 13 épitaphes d'étrangers; mais nous pouvons montrer depuis longtemps que la stèle $\mathrm{n}^{\circ} 64$, primiti-

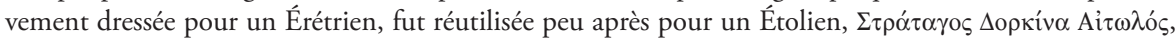
cet ethnique ayant été pris à tort pour un nom propre par l'éditeur.

190. Il y a 188 numéros dans Chr. Dunant (n. 55). Le soussigné avait cependant pu indiquer à l'éditrice, au moment de la correction des épreuves, que le fragment $\mathrm{n}^{\circ} 131$, trouvé dans le Canal devant l'enceinte, pouvait être raccordé au $\mathrm{n}^{\circ} 169$, stèle pour un citoyen d'Amisos, remployée dans l'Édifice I, observation dont Chr. Dunant put tenir compte; même chose pour les nos 44 et 102, qui se raccordent parfaitement. Par ailleurs, le chiffre de 25 prend en compte non seulement le $\mathrm{n}^{\circ} 1$, épigramme pour un

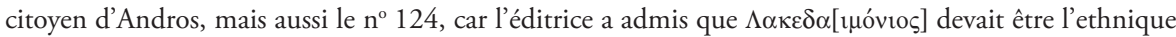
bien plutôt que le patronyme du défunt Dardanos; il faut écarter en revanche la stèle $\mathrm{n}^{\circ} 24$ pour un personnage dont l'appartenance au corps civique érétrien a pu paraître douteuse à cette épigraphiste (cf. ibid., p. 25) en raison de la couleur nettement béotienne du nom $\Delta \varepsilon v^{\prime}$ á $_{\text {: }}$ : mais ce motif d'exclusion n'est certainement pas suffisant. 
déjà été un peu à l'écart ou qu'elle ne servait déjà plus à l'extrême fin du $\mathrm{III}^{\mathrm{e}}$ s. ${ }^{191}$, quand le nombre des mercenaires étrangers au service de la Macédoine dut s'accroître en raison du conflit engagé par les Romains contre les possessions du roi Philippe V.

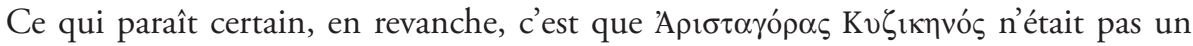
mercenaire au service de la Macédoine, car, au vu de la gravure et d'abord du support, cette belle stèle à fronton sculpté en relief mais non découpé ${ }^{192}$ ne saurait être postérieure au $\mathrm{IV}^{\mathrm{e}}$ s., époque à laquelle il n'y avait pas encore de garnison fixe à Érétrie. C'est, à notre connaissance, le second citoyen de Cyzique à avoir péri en Eubée : l'épitaphe d'un

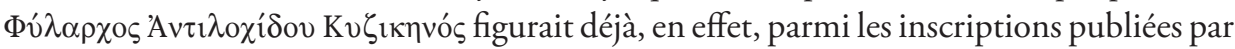
Chr. Dunant ${ }^{193}$. On ne s'étonne pas que cette grande cité commerçante ${ }^{194}$ ait entretenu des relations avec les cités du Canal euboïque : un proxène de Cyzique est d'ailleurs attesté à Histiée, sinon encore à Érétrie ${ }^{195}$. Quant au nom porté par notre Cyzicénien, il est des plus caractéristiques de cette vieille colonie de Milet et de sa métropole ionienne, car on sait qu'un tyran du nom d'Aristagorès ${ }^{196}$ exista non seulement à Milet mais à Cyzique même aux alentours de 500; dans les deux villes les exemples de cet anthroponyme sont, de fait, nombreux sous la forme commune qu'il présente ici ${ }^{197}$. Si le patronyme du personnage n'est pas indiqué, c'est peut-être parce qu'il n'était pas connu - comme on peut assez aisément l'admettre ${ }^{198}$ - des personnes qui, à Érétrie, prirent soin de faire graver une stèle sur la tombe de cet étranger décédé bien loin de chez lui. Mais ce pourrait être dû, tout aussi bien, à l'usage - qui prévalait encore à cette date et plus tard -

191. Voir supra, p. 204, et la conclusion p. 223.

192. Ce type dit II C’ chez Chr. Dunant (n. 55), p. 22, fait son apparition à Athènes au milieu du Iv s. Pour ce qui est de son usage à Érétrie, on en précise la chronologie à propos d'un décret érétrien de proxénie publié, avec deux autres, dans REA 119 (2017) p. 395-484.

193. Chr. Dunant (n. 55), no 176; cf. SEG XXVIII 725, et Bull. ép. 1979, 349.

194. Sur cette ville dont l'exploration et l'étude ont longtemps été contrariées par des conditions peu favorables à la recherche, voir maintenant l'ouvrage collectif édité par M. SÈve et P. Schlosser, Cyzique, cité majeure et méconnue de la Propontide antique (2014).

195. Voir la liste IG XII 9, 1187, 1. 32, récemment rééditée par W. MACK, Polis and Proxeny. Institutional Networks in the Ancient Greek World (2015), p. 316-317 n 8, avec la carte en p. 157. Il est surprenant que parmi les très nombreuses proxénies d'Oropos il n'y en ait aucune pour un Cyzicénien, alors qu’y apparaissent des représentants d'autres cités de la Propontide et du Pont.

196. Connus par Hérodote, tant à Cyzique (LGPNV.A, s.v. $\mathrm{n}^{\circ} 1$, patronyme inconnu) qu’à Milet (V.B, s.v. $\mathrm{n}^{\circ} 1$, fils de Mo $\lambda \pi \alpha \gamma_{0} \rho \eta \varsigma$, autre nom typiquement milésien : cf. supra, n. 181).

197. LGPN V.A, s.v. $\mathrm{n}^{\text {os }}$ 34-43 (y compris le territoire cyzicénien et la ville de Daskyleion).

198. La chose s'observe assez souvent, en effet, dans les épitaphes pour les étrangers comme pour les étrangères : dans le lot publié en 1978, il y a 16 cas sur 25 (cf. Chr. Dunant [n. 55], p. 23), et le rapport est à peu près le même dans IG XII 9. 
de n'inscrire que le seul nom du défunt ${ }^{199}$. Un autre monument funéraire érétrien paraît riche d'enseignement à cet égard : c'est la stèle - parvenue au Musée d'Érétrie vers $1980^{200}$

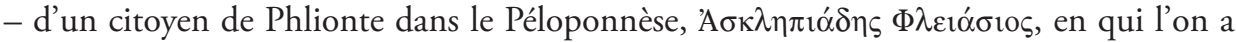
pu reconnaître avec une quasi certitude ${ }^{201}$ (compte tenu aussi de la date approximative de l'inscription) le philosophe Asklépiadès - précisément natif de Phlionte - qui fut l'alter ego de Ménédème d'Érétrie et qui, au témoignage de Diogène Laërce, «mourut à Érétrie avant son ami à un âge avancé» (Vies II 138), sans doute vers 270. Or, en pareil cas, on ne peut douter que le patronyme du défunt (non attesté par ailleurs) fût connu du philosophe érétrien : c'est donc délibérément, pour se conformer à un usage ancien alors déjà en voie de disparition, que celui-ci prit le parti de ne faire graver, sur la stèle de son ami ainsi décédé en terre étrangère, que l'idionyme accompagné de l'ethnique.

\section{CONCLUSION}

Les découvertes fortuites résultant des pluies torrentielles qui se sont abattues à Érétrie à l'automne 2009, si partielles et délicates à interpréter soient-elles, ont néanmoins une portée qui dépasse le secteur des investigations limité au Nord-Ouest de l'antique agglomération. Elles éclairent d'un jour nouveau différents aspects de la cité, touchant à la dynamique du paysage et à des questions d'urbanisme et de société.

L'insignifiant lit de cours d'eau qui borde aujourd'hui le site à l'Ouest, à sec la majeure partie de l'année, est pourtant à l'origine de la plaine alluviale sur laquelle s'est développé le site dans l'Antiquité. Son endiguement dans la première moitié du VII ${ }^{\mathrm{e}} \mathrm{s}$. est sans doute l'un des premiers actes d'envergure de la communauté naissante. Son tracé marque dès la fin de l'époque archaïque la limite entre la cité des vivants, retranchée à l'Est derrière ses fortifications, et les grandes nécropoles, qui s'étendent à l'Ouest du pied de l'acropole à la côte.

199. Parmi les inédits, on trouve surtout des épitaphes du $\mathrm{III}^{\mathrm{e}} \mathrm{s}$. avec patronyme (ainsi pour un Ténédien, un Locrien, un Magnète, un Thessalien, un Cassandréen, un Thrace, un Crétois, un Étolien; cf. supra, n. 189), mais aussi quelques-uns, plus anciens, sans patronyme (par exemple pour un Syracusain et pour un Argien).

200. Inv. 13454. Elle fut intégrée dès 1983 dans ma thèse d'État soutenue l'année suivante à la Sorbonne, puis mentionnée à propos de Diogène Laërce, II 138 chez D. KNoepfler, La Vie de Ménédème d'Érétrie de Diogène Laërce (1991), p. 193 n. 58.

201. Voir D. Knoepfler, Apports récents des inscriptions grecques à l'histoire de l'Antiquité (2005), p. 28-30 et fig. 4 (cf. SEG LV 970). Cette identification a été adoptée ensuite, avec une réserve de principe, par M. HaAke, "Das Grabstein des Asklepiades Phleiasios in Eretria, Philosoph und Freund des Menedemos von Eretria. Zu SEG LV 970 ", MH 67 (2010), p. 233-237, avec une photo et quelques considérations sur l'évolution de l'épigraphie funéraire érétrienne (cf. Bull. ép. 2011, 334; SEG LX 972). 
Les tronçons de murs du canal et de la fortification mis au jour dans les berges ravinées complètent le plan d'ensemble du site. Ils laissent entrevoir l'importance du secteur Nord, situé à l'entrée du ruisseau dans la plaine et où l'on restitue une des principales portes de la ville. Les vestiges d'un pont qui permettait d'enjamber le cours d'eau à cet endroit ont pu être mis en évidence. Il bordait un espace funéraire en fonction dès la fin du $\mathrm{v}^{\mathrm{e}}$ s. et jusqu'au tournant de l'ère chrétienne. Les tombes qu'accueillait cet espace, ainsi que celles révélées plus au Sud au-delà de la Porte de l'Ouest, si fragmentaires soientelles, complètent néanmoins notre connaissance des nécropoles érétriennes, relativement méconnues en raison des pillages survenus à la fin $\mathrm{du}_{\mathrm{XIX}}{ }^{\mathrm{e}} \mathrm{s}$. L'étude complémentaire des 29 stèles funéraires retrouvées dans le lit du cours d'eau donne un éclairage bienvenu sur la société érétrienne des époques classique et hellénistique. Ces inscriptions s'ajoutent aux dizaines de stèles récupérées dans les fouilles suisses aux abords de la porte de la ville et en remploi dans le quartier d'habitations et témoignent de la désaffection d'une partie de la nécropole occidentale dans le cours du $\mathrm{II}^{\mathrm{e}}$ s. Il est tentant, à titre d'hypothèse, de mettre en relation ces bouleversements avec le siège de la ville par les Romains et leurs alliés en 198. Mais sans doute ce phénomène fut-il amorcé à l'extrême fin du III ${ }^{\mathrm{e}}$ s. déjà ${ }^{202}$. En effet, les transformations apportées à la Porte de l'Ouest à cette période (selon toute vraisemblance avant le siège) semblent avoir modifié la circulation à l'entrée de la ville. La nouvelle plateforme de tir enjambant le canal grâce à son beau passage voûté devait restreindre le trafic et peut-être l'interdire épisodiquement ${ }^{203}$. De surcroît, l'importance militaire de ce secteur, qui abritait assurément de coûteuses machines de tir, imposait des règles de sécurité nouvelles, notamment pour se prémunir contre les tentatives de sabotage ${ }^{204}$. Tout porte à croire que la Porte de l'Ouest devint alors un secteur sécurisé qui, sans pour autant interdire tout trafic, en restreignait considérablement le volume au point de dévier chariots, piétons et animaux vers d'autres portes urbaines situées à proximité ${ }^{205}$. Ces différents facteurs, auxquels s'ajoutent les événements désastreux de 198, expliquent probablement le délaissement progressif de ce secteur au $\mathrm{II}^{\mathrm{e}}$ s. et son manque d'attrait comme zone d'ensevelissement. Il faudra confronter ces conclusions aux découvertes futures qui, espérons-le, se réaliseront dans des conditions moins dramatiques que celles qui entourèrent la fouille de sauvetage du canal et permettront d'aboutir enfin à une véritable synthèse sur les nécropoles érétriennes.

202. Voir ci-dessus, p. 171 et 204.

203. Sur les deux passages latéraux prévus, un seul (au Sud) fut réalisé ultérieurement. Ces nouvelles conditions venaient assurément compliquer les multiples entrées et sorties quotidiennes des chariots, charrettes, et autres mules, puisque tout croisement était devenu impossible. Sur les modifications de la Porte de l'Ouest à cette époque, voir C. Krause (n. 12), p. 50-58, et S. Fachard (n. 8), p. 105-107.

204. Les menaces de sabotages sont courantes aux portes des villes, voir Énée le Tacticien, Poliorcétique XVIIIXX et XXVIII.

205. On pense à la Porte du Nord, dont il a été question ci-dessus, et à la porte qui se trouve dans la cour du Musée (fig. I, en $\mathrm{AB} / 800$, voir ci-dessus n. 43). 
"Eretria, the city of tombs». Ce sont en ces termes que les premiers fouilleurs américains du site surnommèrent la ville, frappés qu'ils étaient par la quantité de tombes et de tombeaux intacts qui se trouvaient alors dégagés ${ }^{206}$. Après plus d'un siècle de recherches, qui font désormais d'Érétrie la ville antique la mieux connue d'Eubée - et même l'une des mieux connues de Grèce propre -, les nécropoles demeurent relativement mal délimitées et documentées, faute de fouilles systématiques de grande envergure en dehors des murailles. La fouille du Canal, malgré son caractère restreint, a néanmoins livré un riche matériel épigraphique et des données nouvelles concernant la topographie de la ville antique et de ses nécropoles. Elle nous rappelle combien le monde des morts, bien que rejeté en dehors des murs, faisait partie intégrante du paysage urbain des villes grecques.

206. R. B. Richardson, «Inscriptions Discovered at Eretria», AJA First Series VII (1891), p. 247. Voir aussi J. Pickard (n. 39), p. 389. 


\section{ANNEXE}

\section{INDEX ONOMASTIQUE}

(en gras les noms fournis par les 29 stèles publiées ici, avec leur numéro dans le catalogue entre parenthèses; en maigre les autres noms allégués dans la discussion)

AAnvalis n. 90

Apıóv $\theta$ เos n. 167

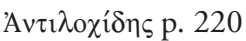

Ал $\alpha \dot{\lambda} \lambda \eta(2)$ p. 211

"А $\pi \alpha \lambda \circ \varsigma$ p. 211

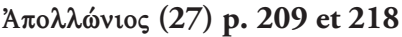

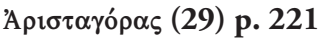

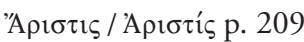

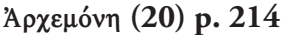

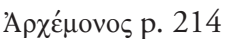

Apí $\sigma \tau \alpha \rho \chi \varsigma$ (3) p. 218

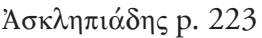

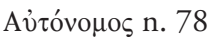

Bı́́ $ๆ$ (21) p. 214

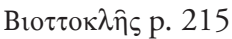

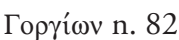

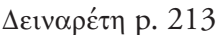

$\Delta \varepsilon$ sivías n. 190

$\Delta \varepsilon \xi \alpha \rho \varepsilon ́ \tau \alpha$ n. 146

$\Delta \varepsilon \rho \kappa \nu \lambda i \delta \emptyset \varsigma$ р. 214

$\Delta \eta \mu \alpha \rho \dot{\varepsilon}$ р р. 213

$\Delta \eta \mu \eta \tau \rho^{i} \alpha$ (4) p. 214

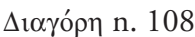

$\Delta$ óкıно (5) p. 217

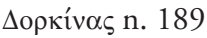

$\Delta$ opv́ $\alpha$ os p. 218

$\Delta \omega$ pló́ (22) p. 210

$\Delta \omega{ } 1 \varepsilon v ́ \varsigma$ p. 210

'Е $\mu \pi \varepsilon \delta$ ovíкๆ (6) p. 212

Eưßı⿻ (20) p. 218

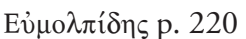

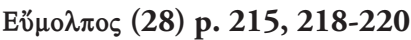

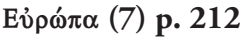

'Hүnpíns p. 206

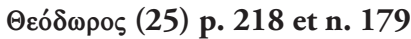

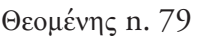

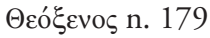

$\Theta \varepsilon \sigma \sigma \alpha \lambda \operatorname{só}_{\text {sp. }} 211$

$\Theta \varepsilon \tau \tau \alpha \lambda \eta \dot{~(1) ~ p . ~} 211$

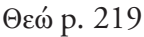

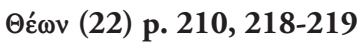

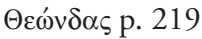

"ISiov n. 117

$\mathrm{K} \alpha \lambda \lambda$... (23) n. 188

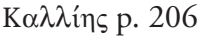

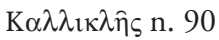

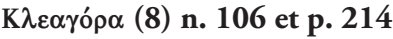

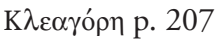

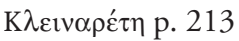

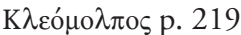

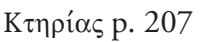

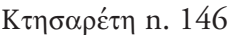

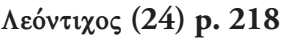

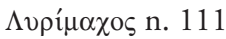

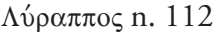

$\Lambda v \sigma i ́ \mu \alpha \chi 0 \varsigma$ (25) p. 208, 218

Ma... (23) n. 188

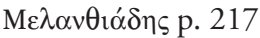

Mع $\lambda \alpha \dot{v} v \theta 10 \varsigma$ (18) p. 217

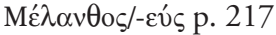

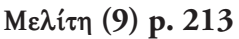

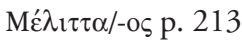

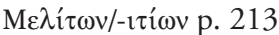

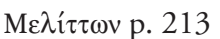

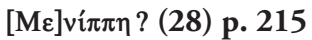

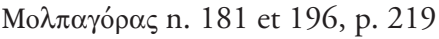




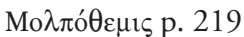

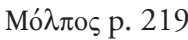

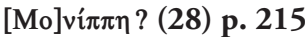

Mví̄oкоక n. 120

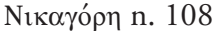

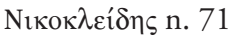

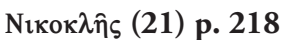

Nıкокро́тпৎ n. 79

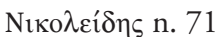

Nıкú $\lambda \lambda \alpha$ (26) p. 215

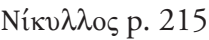

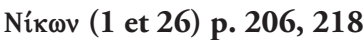

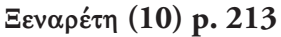

[ $\Xi \varepsilon]$ ví $\pi \pi \eta ?$ (28) p. 215

$\Xi \varepsilon v o ́ \varphi ı \lambda \circ \varsigma(11)$ n. 144 et p. 218

'Ovท́рцио р. 207

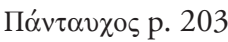

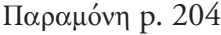

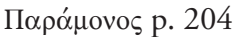

П $\alpha \varepsilon^{\prime} \delta \eta \varsigma$ n. 70

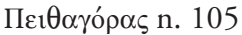

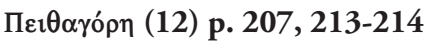

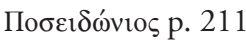

$\Sigma \uparrow \lambda \alpha v o ́ \varsigma$ n. 119

$\Sigma \imath \lambda \alpha v i ́ \omega v$ n. 119

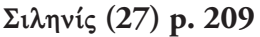

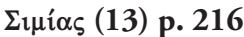

Eîuov n. 117

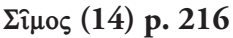

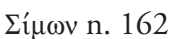

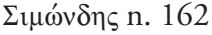

$\Sigma \tau \rho \alpha ́ \tau \alpha \gamma о \varsigma$ ก. 189

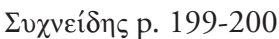

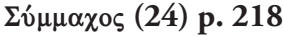

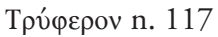

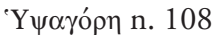

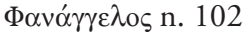

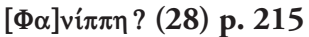

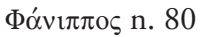

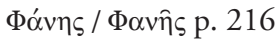

Фâvos (15) p. 216

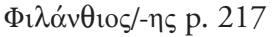

Фú $\lambda \alpha \rho \chi 0 \varsigma$ p. 221

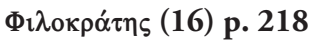

$\Phi 1 \lambda \circ \xi_{\varepsilon} \varepsilon v /-\xi_{\varepsilon v o \varsigma}$ p. $212-213$

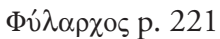

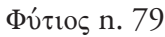

X $\alpha \rho ı \delta \alpha ́ \mu \alpha$ n. 124

Харі́нолтољ р. 219

Xарîvos (17) p. 216

Харías n. 166

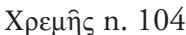

Ethniques

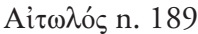

A $\chi \alpha$ ï̌s p. 215

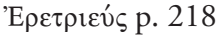

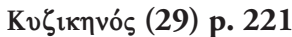

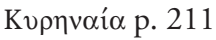

$\Lambda \alpha \kappa \varepsilon \delta \alpha u$ óvios n. 190

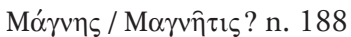

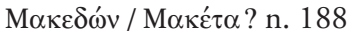

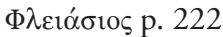

